\title{
A Framework and Review of Customer Outage Costs: Integration and Analysis of Electric Utility Outage Cost Surveys
}

\author{
Prepared for \\ Imre Gyuk \\ Energy Storage Program \\ Office of Electric Transmission and Distribution \\ U.S. Department of Energy \\ by \\ Leora Lawton, Michael Sullivan, Kent Van Liere, and Aaron Katz \\ Population Research Systems, LLC \\ and \\ Joseph Eto \\ Lawrence Berkeley National Laboratory
}

November, 2003

The work described in this report was funded by the Office of Electric Transmission and Distribution, Energy Storage Program of the U.S. Department of Energy under Contract No. DE-AC03-76SF00098. 


\begin{abstract}
A clear understanding of the monetary value that customers place on reliability and the factors that give rise to higher and lower values is an essential tool in determining investment in the grid. The recent National Transmission Grid Study recognizes the need for this information as one of growing importance for both public and private decision makers. In response, the U.S.

Department of Energy has undertaken this study, as a first step toward addressing the current absence of consistent data needed to support better estimates of the economic value of electricity reliability. Twenty-four studies, conducted by eight electric utilities between 1989 and 2002 representing residential and commercial/industrial (small, medium and large) customer groups, were chosen for analysis. The studies cover virtually all of the Southeast, most of the western United States, including California, rural Washington and Oregon, and the Midwest south and east of Chicago. All variables were standardized to a consistent metric and dollar amounts were adjusted to the $2002 \mathrm{CPI}$. The data were then incorporated into a meta-database in which each outage scenario (e.g., the lost of electric service for one hour on a weekday summer afternoon) is treated as an independent case or record both to permit comparisons between outage characteristics and to increase the statistical power of analysis results.
\end{abstract}

Unadjusted average outage costs and Tobit models that estimate customer damage functions are presented. The customer damage functions express customer outage costs for a given outage scenario and customer class as a function of location, time of day, consumption, and business type. One can use the damage functions to calculate outage costs for specific customer types. For example, using the customer damage functions, the cost experienced by an "average" customer resulting from a 1 hour summer afternoon outage is estimated to be approximately $\$ 3$ for a residential customer, $\$ 1,200$ for small-medium commercial and industrial customer, and $\$ 82,000$ for large commercial and industrial customer. Future work to improve the quality and coverage of information on the value of electricity reliability to customers is described. 


\section{Acknowledgement}

The authors thank Imre Gyuk, DOE Energy Storage Program, and Tina Kaarsberg ${ }^{*}$ for their support of this project. We also thank the utilities that agreed to provide the data used in the report; however, by agreement, we do not identify these firms individually. We also acknowledge the assistance of Ram Adapa, EPRI, in obtaining one of the data sets. A draft of this report benefited greatly from expert reviews provided by Robin Walther, George Gross, Ali Chowdhury, Reed Edwards, Diane Barney and members of the NARUC Electricity Reliability Staff Subcommittee, Harry Stoller, Michael Sheehan, Frank Harris, and Bernard Neenan. All errors and omissions remain the responsibility of the authors.

\footnotetext{
${ }^{*}$ Ms. Kaarsberg is currently staff to the Energy Subcommittee of the Committee on Science, U.S. House of Representatives.
} 


\section{Table of Contents}

Abstract

Acknowledgment _ _ _ ii

Table of Contents _. _ n iv

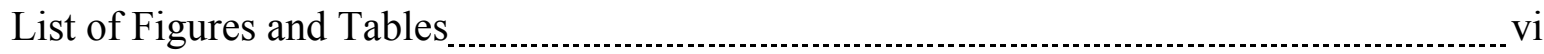

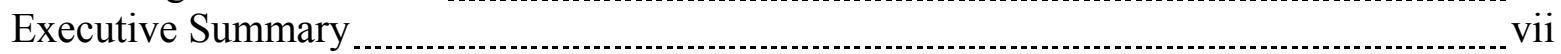

1. Introduction

2. Development of an Integrated Data Base

2.1 Overview

2.2 Commercial and Industrial Datasets $\ldots \ldots \ldots \ldots$

2.3 Residential Data $\ldots$

3. Large Commercial and Industrial Results

3.1 Customer Damage Function Estimation

4. Small-Medium Commercial and Industrial Results $\ldots$

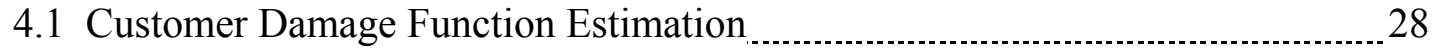

5. Residential Results

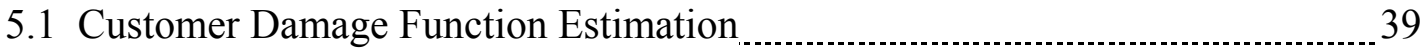

6. Summary and Conclusions

References

Appendix A. Data Transformation

A.1 Acquiring the Datasets

A.2 Construction of the Database

A.3 Missing Data and Treatment of Outliers - C\&I

A.4 Calculation of Total Outage Costs - C\&I .................................................. 54

A.5 Calculation of Willingness to Pay and Credit - Residential ...............................5

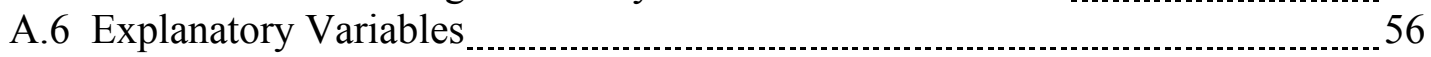

A.7 Dollar Standardization $\ldots$

Appendix B. Value of Service Survey Methodology _.............................................. 59

B.1 Survey-Based Methods of Cost Estimation $\ldots$

B.1.1 Direct Cost Estimation $\ldots$

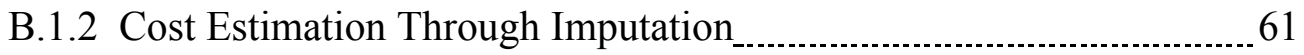

B.1.3 Survey Design

B.2 Data Collection Methodology $\ldots$

B.2.1 Non-Residential Customers

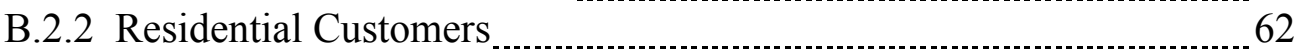


Appendix C. Recommendations for Questionnaire Design _......................................... 63

C.1 Macro- Versus Micro-Views $\quad 63$

C.2 The Impact Of Back-up Systems

C.3 Advance Warning

C.4 Facilitating Regional Comparisons

C.5 Commercial and Industrial Classification Codes (NAICS or SIC) _................. 64

C.6 Residential Costs and Presence at Home

Appendix D. Supporting Documents 65

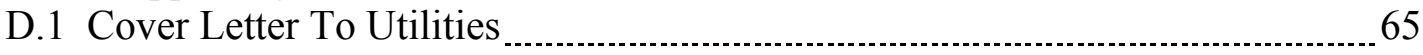

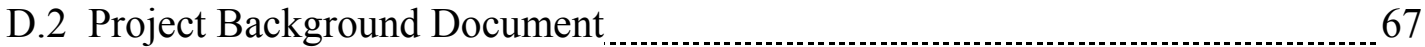

D.3 Project Objectives $\quad 70$

D.3.1 Extend Database On Costs of Outages and Power Quality

Problems $\quad 70$

D.3.2 Synthesize Customer Damage Functions _................................... 71

D.3.3 Benefits to Utility Stakeholders

D.3.4 Analysis of Risks to Utility Stakeholders

D.3.5 Competitive Disadvantage ............................................................. 73

D.3.6 Risk from Litigation

D.4 Description of Information Requested From Utility Participants _.................... 76 


\section{List of Figures and Tables}

Figure 3-1. Logged Per Event Cost Distribution

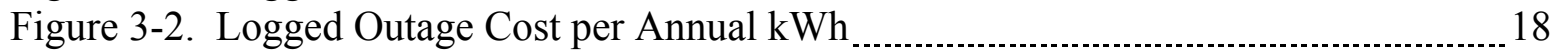

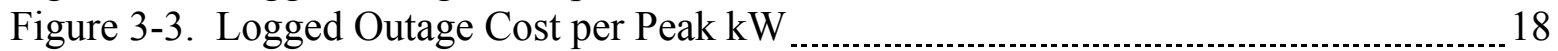

Figure 3-4. Customer Damage Functions - Model One Varying Season and

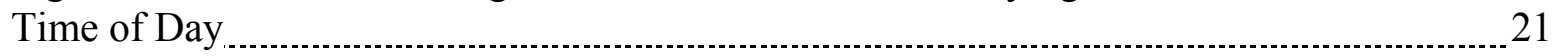

Figure 3-5. Customer Damage Functions - Model One Varying Customer Size _............... 22

Figure 3-6. Customer Damage Functions For Various Business Types _.......................... 23

Figure 4-1. Logged Per Event Cost - Small-Medium C\&I _ _..................................... 29

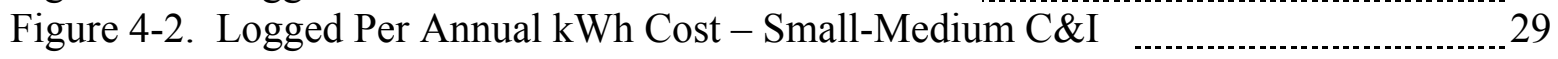

Figure 4-3. Logged Per Peak kW Cost - Small-Medium C\&I _................................... 30

Figure 4-4. Customer Damage Functions - Model One Varying Season and

Time of Day

Figure 4-5. Customer Damage Functions - Model One Varying Customer Size _............... 33

Figure 4-6. Customer Damage Functions For Various Business Types _............................ 34

Figure 5-1. Predicted Outage Cost By Region and Duration _....................................... 41

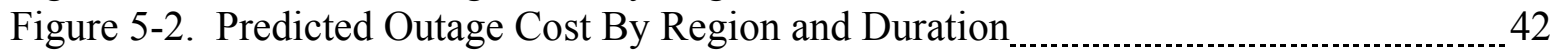

Figure 5-3. Predicted Outage Cost By Region and Duration $\ldots \ldots \ldots \ldots$

Table 3-1. Number of Cases by Region, Company, Season and Day of Week

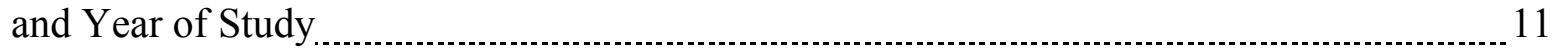

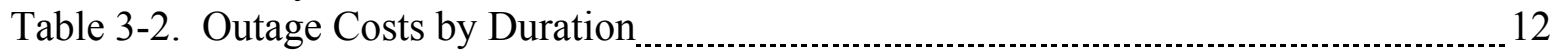

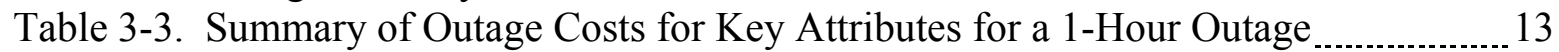

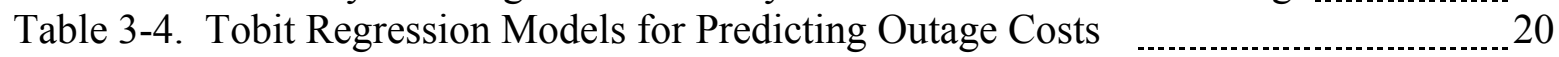

Table 4-1. Number of Cases by Region, Company, Season and Day of Week -

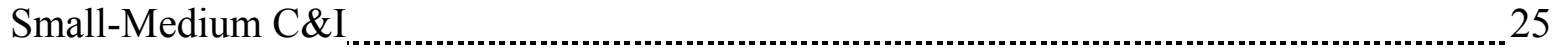

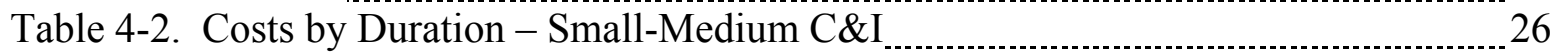

Table 4-3. Summary of Outage Costs for Key Attributes for a 1-Hour Outage ......................... 27

Table 4-4. Tobit Regression Models - Interruption Costs for Small-Medium C\&I ................ 31

Table 5-1. Number of Responses by Region, Season, Day of Week, and Year .................... 36

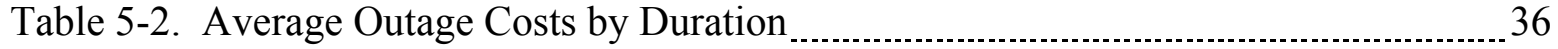

Table 5-3. Average Outage Costs for Key Variables (WTP and WTA) _........................... 38

Table 5-4. Tobit Regression for the Residential Data, Willingness-To-Pay ...................... 40

Table 6-1. Summary of Studies Used in the Meta-analysis _...................................... 45

Table 6-2. Summary of Predicted Outage Cost $\ldots . \ldots . \ldots$

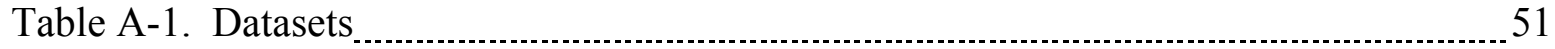

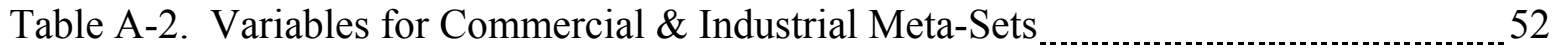

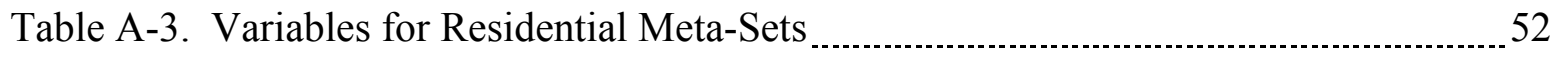

Table A-4. Sample Sizes of C\&I Analysis Datasets Before and After Deletion of Outliers

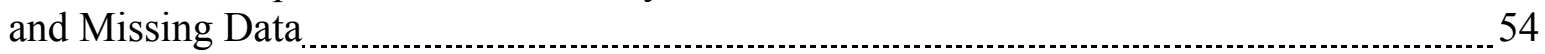

Table A-5. Sample Sizes of Residential Analysis Datasets Before and After Deletion of

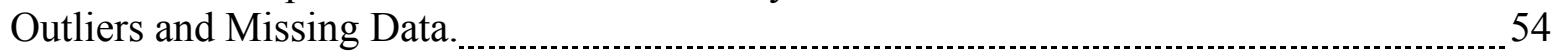

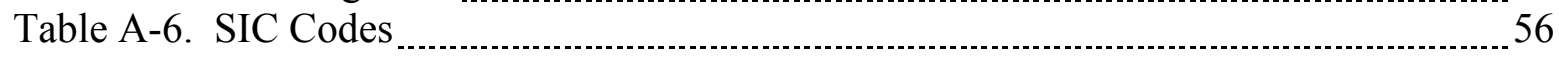




\section{Executive Summary}

Ensuring reliability has and will continue to be a priority for electricity industry restructuring. Indeed, the recent blackout in the Northeast highlights the significant public and private interest in electricity reliability. A key aspect of having electricity available on-demand, whether it is to individual households or large industrial complexes, is the fact that outages - brief or extended interrupt essential as well as discretionary use of appliances, motors, electronics and other devices for which electricity is the primary, if not the only, source of energy.

Ensuring reliability, however, is a complex and multi-faceted problem that necessarily involves actions taken by both public and private decision makers. Strategies to provide reliable service are numerous, and some carry hefty price tags. Overbuilding the entire electricity delivery system to reach a standard of reliability that costs more than consumers are willing to pay or under-building the system that leads to more outages than customers are willing to bear are both sub-optimal strategies. It is, therefore, important to understand what reliability costs consumers as well as how much they are willing to pay for reliability, so that appropriate public and private investments and operating decisions can be undertaken.

Over the last 20 years, there have been numerous efforts to quantify the value of reliability as a basis for both public policy and private investment and operating decisions. These efforts undertaken primarily by electric utilities seeking to better understand the value of electric service (VOS) by conducting customer interruption or outage cost studies are expensive. Consequently, only a few studies have been conducted and, individually, they are of limited usefulness for understanding the value of reliability for consumers in other regions or under different outage scenarios. The solution taken by this study is to combine findings from available outage studies into a meta-dataset and extract findings that increase the availability and applicability of findings beyond the scope of the original studies. Merging findings from individual studies and extrapolating to develop new finding in this manner is facilitated by the fact that the data were originally collected using a common methodology, which is documented in Sullivan and Keane's (1995) Outage Cost Estimation.

\section{Methodology}

The process to acquire, standardize, and merge the various datasets was straightforward. Ten of the twelve companies that had conducted VOS studies during the past two decades were contacted. Eight agreed to participate. Data files, questionnaires and codebooks were obtained; the data were then standardized and merged together. Dollar values were standardized by adjusting them to 2002 dollars using the Consumer Price Index. Altogether, 24 independent datasets were merged into three meta-datasets: one each for data collected from large commercial and industrial (C\&I), small-medium C\&I, and residential customers. These studies comprised 13 years of experience, using a variety of outage scenarios, and covering large portions of the United States (but not including the Northeast or much of the north-central Midwest). Each dataset included the key dependent variables of outage cost per event for the C\&I customers, and willingness to pay measures per event for the residential data. Other explanatory factors, such as SIC code, firm size, and type of housing, were also included. 
Statistical power was enhanced by organizing the data such that each scenario in a survey was treated as a single record: If, for example, one respondent provided input about 3 different outage cost scenarios, the final data set included 3 records for this respondent instead of one.

\section{Results}

Results were developed in two basic formats: (1) summary (bivariate analysis) tables for various scenario factors and customer characteristics for an outage of one hour, and (2) customer damage functions using multiple regression (Tobit) models for estimating outage costs while controlling for all factors simultaneously. The Tobit models predict that the average cost experienced by an "average" customer for a single summer afternoon outage of one hour is approximately $\$ 3$ for residential, $\$ 1,200$ for small-medium commercial and industrial, and $\$ 82,000$ for large commercial and industrial. The outage costs increase substantially, but not linearly, as the outage duration increases from one to eight hours. Outage costs are generally higher in the winter than in the summer for an outage of a given duration or time of day. The Tobit models also reveal important differences in outage costs across regions, time of day, customer size, and business type.

Use of the data is subject to important caveats. The most important of these is collinearity, which means that the findings are inextricably linked to aspects of the original studies from which they were derived and that, therefore, the extrapolations cannot be fully supported on statistical grounds. In addition, as noted earlier, data on the Northeast and some areas of the Midwest were not available for inclusion in this initial study. Finally, the original studies were not identical in every respect; all variables were not collected consistently by each study.

\section{Conclusions and Recommendations}

The principal contribution of this project has been the development of statistical models that can be used to estimate outage costs for large C\&I, small-medium C\&I, and residential customers. These models can be applied to estimate outage costs for representative customers in different geographic regions for a variety of outage scenarios. Utility planners, for example, can use the results to estimate outage costs for particular customer classes (or mixes of customer in different classes) representative of their service territory's customer base. They can, thereby, improve generation, transmission and distribution planning processes compared to processes that do not consider the economic value of reliability to consumers.

This study is an initial effort to improve the public availability of information on the economic value of reliability. More work is needed to improve the quality and coverage of this critically important information. In particular, to address some of the limitations in the current data, we recommend the following:

1. Encourage all U.S. utilities that have conducted surveys on the economic costs of outages to their customers to contribute these data and thereby enhance the coverage and usefulness of these data on a national scale

2. Support future utility efforts to collect additional information on the value of electricity reliability toward ensuring that ultimately these data also contribute to improving the 
availability of this type of information on a national basis (e.g., use consistent methodology for survey design and sampling).

3. Where necessary, in order to address existing gaps in available data, lead efforts to conduct additional surveys on topics of high priority (e.g., collect new data in areas of the country or on other reliability issues not currently well-represented in existing data collection efforts or on other reliability topics. 


\section{Introduction}

Ensuring reliability has and will continue to be a priority for electricity industry restructuring. Reliable electric power delivered on demand is a cornerstone of electricity's ubiquitous adoption and use. A central feature in electricity's value to consumers, whether they are individual households or large industrial complexes, is the infrequent occurrence of outages or other power disturbances that interrupt the use of appliances, motors, electronics, or any of the other myriad of end uses for which electricity is the primary energy source.

While no one disagrees that customers seek reliable power, ensuring reliability is a complex and multi-faceted problem. The strategies available to meet that goal are numerous and the price tags associated with them vary greatly. Most important of all, reliability has always been a shared responsibility because it is a public good. Therefore, who pays and who benefits from increased reliability has always been an important question for both private and public decision makers. The recent August 14 blackout in the Northeast - the largest blackout in U.S. history - has punctuated the importance of and at the same time difficulty in determining the best strategy or combination of strategies.

Underlying any strategy is assumptions about the value end-use customers place on reliability. During times of crisis caused by either short-term events like the recent massive outage in the Northeast or the rolling blackouts in California in 2001, a common (yet, we believe inappropriate) assumption is that customers will pay almost any price for reliable power. In contrast, during periods of reliable power delivery but accompanied by rising rates or rising taxes, there are frequent charges that the system is being overbuilt and designed to a higher standard of reliability than customers are willing to pay.

A general framework for addressing this planning problem has been the application of valuebased planning (Vojdani, et al., 1995; Dalton, et al., 1995). Value-based planning is designed to match the level of investment in reliability with the economic value of the improvement in reliability. The use of value-based planning requires a method for estimating customers' value of service reliability. Historically, generation, transmission, and distribution systems investments have been planned using arbitrarily defined engineering criteria. With value-based planning, it is assumed that customer preferences for service reliability can be measured and that these preferences can be used to establish economically justified reliability targets for generation, transmission, and distribution investments.

In the application of value-based planning, the value of service reliability to customers has been conceptualized as equal to the economic losses that customers would experience if a given outage occurred. The economic losses experienced by customers as a result of reliability or power quality problems can be described by a Customer Damage Function (CDF) ${ }^{1}$. The general form of a $\mathrm{CDF}$ is:

Loss $=\mathrm{f}\{$ outage attributes, customer characteristics, geographical attributes $\}$.

\footnotetext{
${ }^{1}$ For a discussion of the application of such functions to electric power supply reliability planning see "Prediction of Customer Load Point Service Reliability Worth Estimates in an Electric Power System," L.Goel and R. Billinton, 1994, IEEE Proc.-Generation, Transmission, Distribution, Vol.141, No. 4, July 1994.
} 
The dependent variable of economic loss is expressed as a loss in dollars per event, per $\mathrm{kWh}$ of un-served energy, per $\mathrm{kWh}$ of annual energy consumption or per $\mathrm{kW}$ of annual peak demand. The equation predicts the economic loss from factors that influence outage costs. The outage attributes might include duration, season, time of day, advance notice and day of the week. The customer characteristics could include annual $\mathrm{kWh}$ usage, $\mathrm{kW}$ demand, type of business, type of household, presence of various outage sensitive equipment, presence of backup equipment, and other firmographic or demographic characteristics. Finally geographical attributes might include temperature, humidity, frequency of storms and other geographical conditions affecting economic losses from outages.

Customer damage function's are useful for reliability planning in several ways. First, the customer damage function provides a framework for conceptualizing and estimating the factors that influence customers' outage costs for particular types of outages. Second, the use of a customer damage function allows for analysis of the isolated effects of different attributes of outages such as duration or time of day. Third, it can be used to quantify the economic losses from different electricity system reliability investments by multiplying appropriately defined customer damage functions by the un-served energy expected under different system investment options. These calculations then become the basis for comparing different reliability solutions and evaluating whether the economic benefits to customers are justified by the costs of the investment options.

The use of customer damage functions and value of service reliability estimates applies to many investment decisions facing utility planners, regulators, and policy makers. To compare alternatives in a planning framework, the calculations may focus on the economic costs or benefits of changes in un-served energy, the frequency of key events (like momentary outages or voltage sags), or other aspects of the economic value of reliability. A few examples serve to illustrate: ${ }^{2}$

- Generation planning: As utilities add capacity, the probability of a generation capacity shortfall declines and the cost of un-served energy at the time of peak demand declines. Reducing the amount and hence cost of un-served energy is valuable to customers, the question is whether these benefits outweigh the costs of obtaining them. By analyzing how the benefits from reducing un-served energy are distributed across customer classes and by knowing the economic value of that un-served energy has for different customers, planners can determine whether costs to improve system generation reliability are balanced with the value of the improvement to customers.

- Transmission planning: Transmission planners analyze the reliability of transmission lines to assure sufficient capacity exists to serve customers under different failure contingencies. With value-based planning, the failure scenarios can be examined based on the number and frequency of voltage sags or power quality events they create and the costs to reinforce the system to reduce these power quality problems. By comparing these costs to the economic value to customers of the reduction in power quality problems, decisions can be made as to whether system reinforcement creates sufficient net benefits to justify these added costs. The

\footnotetext{
${ }^{2}$ Detailed examples of the use of outage costs in various generation, transmission, and distribution planning situations are provided in "Outage Cost Estimation Guidebook", M. Sullivan and D. Keane, TR-106082, Electric Power Research Institute, Palo Alto, CA: December , 1995.
} 
customer damage function combined with the estimates of the frequency with which certain events might occur serve as the basis for calculating the economic value of various options.

- Distribution planning: Customers on a distribution circuit can be served with different circuit configurations (e.g., radial, loop, networked). Each configuration varies in its cost to implement and each has different implications for the expected frequency and duration of outages to customers served by these circuits. Planners can compare options by calculating the expected un-served energy from various circuit designs and by examining the types of customers currently on the circuit and forecasted to locate near the circuit through time. They can also compare designs on the likelihood of various power quality problems. Using a customer damage function, the economic value of the reliability improvements can be calculated for specific groupings of customer types and for the specific reliability problems/improvements anticipated for a given circuit. This economic value can be compared to the cost of various options to balance the investment cost with the anticipated benefits.

Value-based planning concepts have been around for 20 or more years. Over this period, there have been numerous studies to quantify the value of reliability as a basis for both public policy and private investment, and for operating decisions regarding generation, transmission, distribution, and retail offerings. Efforts have been made to measure outage costs or value of service using a range of methods and techniques. Despite these efforts, Eto, et al. (2001) note that:

1. There are few estimates of the aggregate cost of unreliable power to the U.S. economy, and the estimates that are available are poorly documented or based on questionable assumptions.

2. Costs of large-scale outage events (e.g., State- or region-wide power outages) are not well documented and are mostly based on natural disasters for which it is difficult to separate costs of electric interruptions from damages caused by other disaster features (e.g., property damage from wind or water).

3. Studies of hypothetical outages obtained from outage cost surveys could be used to prepare aggregate estimates of outage costs. However, there can be important differences in the survey and statistical methodologies used in the studies that must be addressed in any metaanalysis relying upon them.

4. Very little information is available in the public domain regarding the costs of power quality problems - an increasingly important aspect of service reliability.

This report begins to address this information gap by conducting a meta-analysis of a large number of the large-scale studies conducted by major electric utilities over the past 15 years to assess the value of electric service to their customers. In all, 24 studies were included in this analysis. These studies represent the efforts of 8 electric utilities to measure the value of service reliability or outage cost in the residential, commercial, and industrial sectors. 
These studies were chosen because they employed a common survey methodology including sample designs, measurement protocols, survey instruments and operating procedures. This methodology is described in detail in EPRI's Outage Cost Estimation Guidebook (Sullivan and Keane, 1995). A brief discussion of this methodology can be found in Appendix B. The goal of this project was to assess the feasibility of combining the actual survey data from these independently conducted studies into a single database by assembling andstandardizing them into a national database of customer interruption costs. The database is used to describe variations in interruption costs, including differences in interruption costs by outage duration and time of day/season, as well as by customer type, characteristics, and regional (geographic) differences.

The 24 studies chosen for analysis include virtually all the Southeast, most of the western U.S. (including almost all of California, rural Washington and Oregon, and the largest metropolitan areas in Arizona and Washington), and the Midwest south and west of Chicago. The time frame covered by the studies ranges from 1989 to 2002. Several studies examined interruption costs for similar customer populations (e.g., residential customers) at roughly the same time using nearly identical measurement protocols, but were conducted by utilities located in different parts of the country. In almost all of the studies, detailed demographic and firmographic information was collected from study respondents and incorporated into the database of results.

While each individual study was extensively analyzed by the utility that conducted the study for their own use, until now there have been no efforts to combine the data from the studies into a single database. The value of combining the data and developing a set of meta-models is the prospect of extending the results of the individual studies in several ways::

1. Individual utilities typically represent only one region of the country, whereas a combined dataset may provide an opportunity to evaluate value of service across regions that will include differences in temperature, humidity, energy rates, and regional economic conditions.

2. Customers are heterogeneous, particularly in the commercial and industrial sectors. Combining the data provides additional cases to examine value of service for important subsegments.

3. Most of the studies examined here use a survey method in which customers responded to various outage scenarios. By combining the data across studies, a broader range of scenarios can be used to estimate the impacts of time of day, duration, season, and certain special conditions, such as receipt of advance notice.

4. The studies were conducted over a 15-year period so some comparisons across time may be possible.

Combining the data has several positive features, but there are also limitations with which to contend. First, because the studies were conducted for specific utilities at specific points in time some variables of interest are "collinear" with each other. For example, if there is only one study from a utility in a particular region conducted at a particular time, then it is hard to separate the effects of their unique climate and rates, and their customers' outage experiences. This requires 
caution in interpreting various effects because the data may not be ideally suited to provide independent estimates of the effect of each variable.

Second, the studies chosen for this combined dataset used similar methods for collecting the data but they did not necessarily use identical methods. As a result, it is important to consider that some effects identified in the data may be the result of "methods" effects rather than substantive effects of different variables.

This report is organized in 5 sections following this introduction. Section 2 describes the methods used to create the combined dataset and provides a description of the data. Sections 3 , 4, and 5 present findings, including customer damage functions, for the analyses conducted of the large commercial-industrial, small-medium commercial, and residential sectors, respectively. Section 6 summarizes our findings and discusses areas in need of additional research. Four appendices follow. The first appendix provides a more detailed discussion of the data transformation procedures used to assemble the databases. The second appendix reviews the value of service methodology. The third appendix reviews issues in customer outage cost survey design. The fourth appendix provides additional background on the present study and on the use of findings from this study. 


\section{Development of An Integrated Data Base}

\subsection{Overview}

The major objective of this project was to identify, gather, and combine the data from prior utility value of service or outage cost studies into separate databases of findings for three distinct customer groups: residential, small-medium commercial and industrial (C\&I), and large C\&I. As part of the initial review of past studies, 12 utilities were identified that had measured customer outage costs using survey-based methods for one or more of these three customers groups. Altogether, 24 datasets from 8 companies were ultimately acquired, standardized, and then merged. Each dataset presented certain issues (see Appendix A), but it was possible in most cases to develop rules for converting the data into meaningful datasets based on common questions or common metrics.

The following steps were taken in creating the databases:

1. Contact the utilities that had conducted customer interruption cost (or Value of Service or outage cost) studies;

2. Negotiate agreement(s) to participate in the study, including agreements not to disclose customer-specific information or present information that could be attributed to an individual firm;

3. Obtain the datasets, codebooks, and original survey questionnaires;

4. Standardize each dataset in terms of variable selection and construct;

5. Merge the datasets;

6. Normalizeoutage costs to a common base year, using the 2002 Consumer Price Index; and,

7. Review the data and exclude outliers and other data anomalies.

The core elements of this process are described in this chapter. Additional details are provided in Appendix A.

First, all variables were standardized using a common metrics. For example, some studies may have described the outage duration in hours (e.g., a 1 hour outage) while others may have used minutes (e.g., a 30 or 60 minute outage). In this instance, the results for both studies would be converted to minutes. Although the survey instruments for the various studies may have used slightly different wordings, each study measured the same basic underlying concepts. These included:

1. Attributes of the Outage (e.g., duration, frequency, season, time of day)

2. Summary of Costs (e.g., labor costs, material costs, damage costs)

3. Customer Characteristics (e.g., company size, household income)

In most cases, it was possible to find a common, underlying metric and convert all the responses to that metric. While differences existed, in only one case were these differences so insurmountable that data could not be merged, and were excluded from the meta-dataset. 
Second, all of the scenarios were hypothetical. This is both a strength and weakness of this body of studies. The goal in presenting customers with hypothetical outage scenarios is that they can respond to the same stimulus or a carefully controlled description of a series of outages. This simplifies associating costs and customer characteristics with attributes of outages like duration and time of day. However, because these are hypothetical, customers do not provide actual costs for actual events. They are asked to carefully estimate their costs for the hypothetical situations, regardless of previous outage experiences. We cannot determine, prime facie, the biases inherent in such self-reports of cost estimates associated with hypothetical outage scenarios.

Third, the outage scenarios varied in several ways, including duration of the outages, time of day when outage began and season during which outage occurred. However, many tended to focus on outages associated with system peak conditions. For example, studies conducted in northern climates were focused primarily on winter outages, while those in southern climates were focused primarily on summer outages. Some studies measured interruption costs for momentary outages, while others did not. Some studies measured costs for long outages (i.e., 8-12 hours), while the maximum outage duration was limited to 4 hours in others. The most commonly used outage scenarios involved outages of one- and four-hour durations occurring on summer afternoons. Most of the studies included a common 1-hour outage occurring at time of system peak for all observations.

Fourth, the studies were conducted over a 15-year period. The results from each study are appropriate for the time period during which the data were originally collected. To compare the results across time it was necessary to take account of inflation and changes in the cost of living. Accordingly, all of the cost data have been adjusted to 2002 dollars using the Consumer Price Index.

Fifth, statistical power of the results was enhanced by organizing the data so that the responses for each scenario in a survey were treated as independent observations or records. The strategy used to collect outage cost data in most of these studies involved presenting customers with a series of hypothetical outages and asking them to describe their costs (or to respond to a willingness to pay to avoid their costs) to each one. Each respondent normally provide cost estimates for more than one scenario (in some cases, up to 8 scenarios). Each response to each scenario was treated as an independent response. For example, if one respondent provided separate cost estimates for each of 3 scenarios, then these results were converted into three separate records. The common variables, e.g., firmographic information such as SIC code, were appended to each record.

Three meta-datasets were created for three customer groups: residential, small-medium C\&I ( 1 MW or less) and large C\&I (more than $1 \mathrm{MW}$ ). After combining all of the survey datasets with comparable measurements, the two commercial and industrial datasets include the following information on each observation:

1. Season;

2. Onset time of day;

3. Onset day of week;

4. Outage duration; 
5. Whether advanced warning was received;

6. Customer's region;

7. Year interruption cost study was completed;

8. Estimated interruption cost;

9. Customer's SIC code;

10. Customer's business type;

11. Number of employees;

12. Whether company has back-up generation; and

13. Customer's annual $\mathrm{kWh}$ consumption.

The residential customers' survey included similar outage scenario information (items \#1-7, above) but also included:

1. Willingness to pay measure (WTP);

2. Willingness to accept credit (WTA or Credit);

3. Type of housing;

4. Home ownership;

5. Household income;

6. Whether household has sickbed resident;

7. Whether household uses medical equipment in the home; and

8. Whether household has a home business.

The commercial and industrial, and the residential datasets are also differed from one another in other important respects, as described in the following two sub-sections.

\subsection{Commercial and Industrial Datasets}

Development of commercial and industrial sector databases involved creating separate databases for the large C\&I and small-medium C\&I data. . Each includes enterprises involved in all aspects of commercial and industrial activity as well as government services. Although utilities use slightly different criteria for defining small-medium versus large customer classes, we used a common criteria to assign customers to either small-medium or large C\&I. The small-medium commercial and industrial customer was defined as a customer with less than $1 \mathrm{MW}$ of peak demand. The large C\&I customer was defined as a customer with more than $1 \mathrm{MW}$ of peak demand..

For both commercial and industrial customers, all of the studies employed the same outage cost estimation methodology - direct worth or direct cost estimation (see Appendix C). In the direct worth estimation methodology, customers were asked to estimate the losses they would experience under varying assumptions about the timing, duration and extent of electric outages. In most cases, the estimation involved customers completing a worksheet for each scenario in which they reported various types of costs and various types of savings. These costs and savings were then summed to calculate a net cost of the outage. Customers were generally asked to provide estimates for four to ten scenarios (i.e., combinations of onset time, duration, extent of advance warning, season and day of the week). Thus, these studies produced a range of estimated outage costs for each customer - one for each combination of interruption conditions 
on which they were asked to report. It is not uncommon for some of the customers within a given study to receive one randomly chosen set of outage conditions, while others receive a somewhat different randomly chosen set.

For the two commercial and industrial datasets, the primary dependent variable is total cost of the outage on a per event basis. In most cases, demand and usage information for each customer was also available and, for reporting purposes, was used to express outage cost on a per peak $\mathrm{kW}$ and per annual $\mathrm{kWh}$ basis. However, these expressions of costs were not calculated individually for each record. Rather, they were calculated by dividing the average total cost per outage event for all records with that scenario by the average $\mathrm{kW}$ or $\mathrm{kWh}$ for the customers who responded to that scenario. This method is reasonable because there is often a good deal of measurement error associated with the estimate of $\mathrm{kWh}$ and $\mathrm{kW}$ for the specific site for which the outage cost data is being collected. Survey respondents are asked to assume the outage occurs at a defined location, but because of multiple meters per site and multiple sites per firm (e.g., the corporate headquarters versus the manufacturing facility) it is not always possible to collect usage and demand data for the specific site being referred to in the survey. By dividing the aggregate per event outage cost by the aggregate $\mathrm{kWh}$ or $\mathrm{kW}$, the errors tend to be smoothed and there are fewer issues with large outliers in the underlying data as a result of incorrect usage or demand estimates for a site.

\subsection{Residential Data}

Unlike the commercial and industrial customers where much of the "costs" associated with an outage can be converted into an economic loss based on lost profits or costs over savings, the costs of outages to residential customers are often more intangible. Residential customers tend to describe their costs in terms of the "hassle" or "inconvenience" of an outage rather than in terms of specific labor or material costs. For this reason, most of the residential outage cost studies in this meta-analysis use some form of 'willingness to pay' (the amount the household respondent would be willing to pay in order to avoid an outage of a certain scenario) as the dependent variable (rather than rely on estimation of direct costs). The meta-analysis described here focuses on these 'willingness to pay' measures. In a few instances, the original studies also included some measure of the amount a customer is "willing to accept" in the form of a credit on the customers' bill for the inconvenience of an outage as a measure of value of service reliability. These latter measures of willingness to accept are less widely used so there was insufficient data to build models across the studies. Available results from both forms of outage cost estimation are presented in Section 5. 


\section{Large Commercial and Industrial Results}

The large commercial and industrial dataset is built from 10 studies conducted by 8 companies and includes approximately 2,009 respondents. Overall there are 8,462 total responses available for the analysis. The number of cases varies, depending on availability of data (that is, either the study or the respondent's scenario details may have missing values). The distribution of the available data across various outage attributes, years, and customer characteristics is described first. A summary of the multivariate analysis is presented second.

In terms of coverage, Table 3-1 summarizes the number of records available for analysis by region, season, day of week, and year of study. Overall there are 7,862 responses to various scenario combinations across these four variables. The results show that the number of responses ranges from 40 to nearly 2000 for various combinations. Overall there is fairly good coverage across regions, for winter versus summer seasons, and across year of study. For the large commercial and industrial sector, there is limited data on weekday versus weekend outages.

While suggesting a reasonable degree of coverage for conducting the meta-analysis, the results in Table 3-1 also point to key limitation in the data. In particular, the results show that there are certain "holes" in the coverage that will limit the ability to use the merged data to sort out effects for some variables. For the four variables in Table 3-1, for example, the region of the country and the year of the study are highly correlated. In most years only one or two utilities did a study and these studies were done in different parts of the county. As a result, if a calculation of the average outage cost for a given year is calculated it would be heavily influenced by the region and type of scenarios asked in that region. For this reason, the data cannot be used very effectively to evaluate the changes in outage costs over time without additional statistical controls for the region (or utility) and scenario characteristics.

Table 3-1. Number of Cases by Region, Company, Season and Day of Week and Year of Study

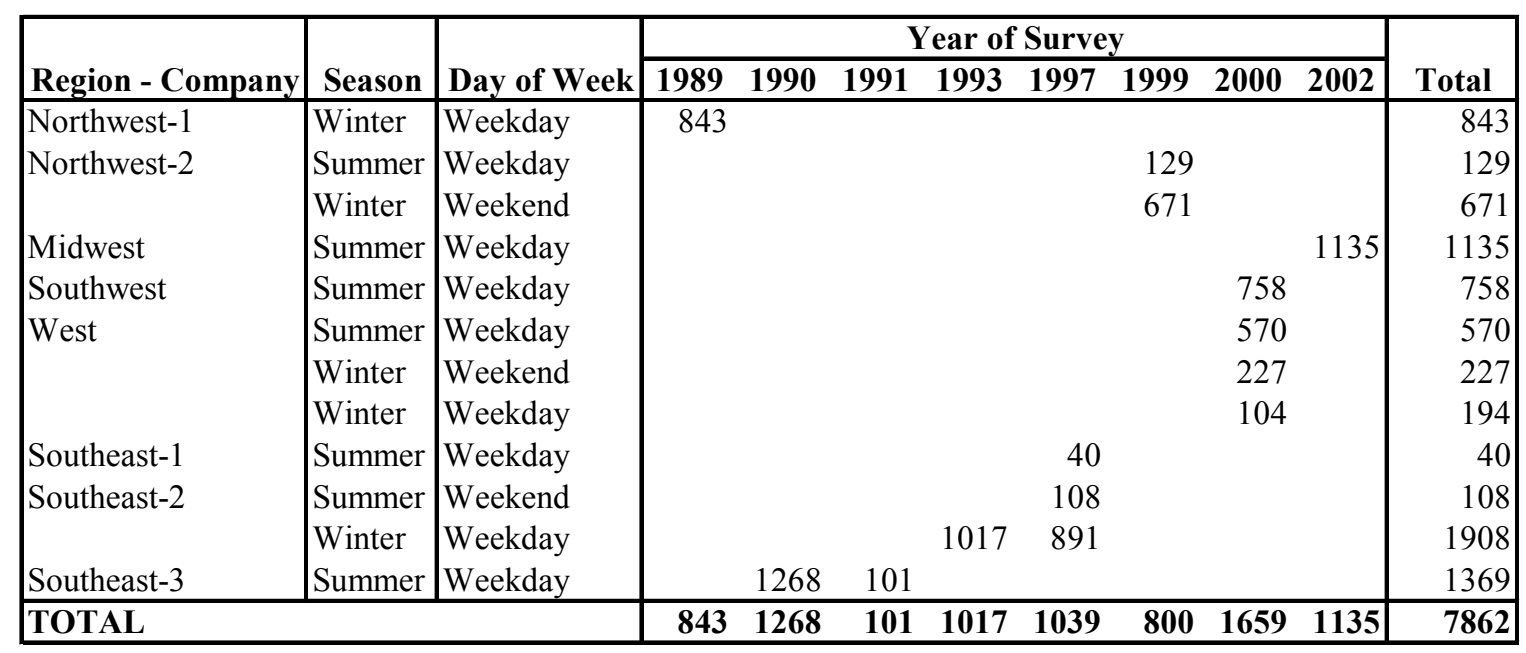

This problem surfaces for many of the calculations of outage costs that would be of interest. Simple comparison of average outage costs for levels of a variable of interest (such as outage costs for different outage durations or for different regions) must be interpreted very cautiously 
outside the context of a multivariate model that can control for other customer or outage attributes. The underlying group of customers responding to a scenario will vary from scenario to scenario and differences in these underlying groups may be more important in explaining differences in the outage costs than the levels of the variable of interest (such as duration).

For this reason, we remind the reader that the regression analysis presented at the end of this chapter provide the most meaningful information on the value of service. The bivariate tabulations presented in the tables are suggestive, but due to the methodological and data structural issues, may be somewhat misleading. For example, it makes sense to compare the effect of a specific condition on outage cost when the same respondents provide information to both permutations. However, frequently one group of respondents provides information about one kind of scenario, and these results are compared to different respondents. Importantly, only regression or similar analyses take all of these factors into consideration simultaneously and consistently.

In Tables 3-2 and 3-3, comparisons of the average outage costs for a 1-hour outage for several key variables — outage duration, season, day of week, region, and SIC grouping - are presented. The data include the mean and median outage cost per event, the standard deviation of the mean, the values representing the 25 percent and 75 percent values around the mean, and the outage cost per event standardized to outage cost per annual $\mathrm{kWh}$ and per $\mathrm{kW}$. These values are presented to provide a measure of the typical values and range of values in the underlying data used in the meta-analysis. These comparisons provide a measure of the validity of the data. However, as noted above, these averages must be compared carefully as the underlying pool of customers included in the calculation changes from level to level.

Table 3-2 shows the distribution of outage costs (per event, per annual $\mathrm{kWh}$, and per peak $\mathrm{kW}$ ) by outage duration. The results show outage costs rising from an average of $\$ 15,601$ for a voltage sag to $\$ 119,715$ for a 4 -hour outage. The results trend generally upward as would be expected. There are two deviations from this trend. First, the 1-2 second outage has a significantly higher per event cost $(\$ 23,097)$ than the events on either side of the duration curve (voltage sag at $\$ 15,601$ and 1-minute outage at $\$ 12,944$ ). The second deviation is the downward trend in outage cost from the 4-hour duration $(\$ 119,715$ per event) to the 8 -hour $(\$ 88,224$ per event) to the 12 -hour duration $(\$ 58,562$ per event). It is possible that these differences represent a methodological artifact as only one study used the 1-2 second duration and the 12-hour duration. A discussion of the effect of duration on outage costs in the context of a multivariate model controlling for differences among the studies is provided later in this section.

Table 3-2 also shows the outage costs converted to costs per annual $\mathrm{kWh}$ and per peak $\mathrm{kW}$. On a per annual $\mathrm{kWh}$ basis, the results also suggest that outage costs generally increase with duration from $\$ 0.0006$ to $\$ 0.0187$ across the range of duration from a voltage sag to a 12-hour outage, respectively. The data on outage cost per $\mathrm{kW}$ of demand is less straightforward. The costs range from $\$ 3$ per $\mathrm{kW}$ of demand for a voltage sag to $\$ 45$ per $\mathrm{kW}$ for an 8 -hour outage. The costs also increase in a generally stepwise fashion but with a large jump for the 15-minute duration. This jump at 15 minutes is likely an artifact of the underlying study that used this duration. 
Table 3-2. Outage Costs by Duration

\begin{tabular}{|c|c|c|c|c|c|c|c|c|c|}
\hline \multicolumn{10}{|c|}{ BY DURATION } \\
\hline & \multicolumn{7}{|c|}{ Total Cost/Event } & $\begin{array}{c}\text { Cost Per } \\
\text { Annual } \\
\text { kWh } \\
\text { Usage } \\
\end{array}$ & $\begin{array}{l}\text { Cost Per } \\
\text { Peak kW } \\
\end{array}$ \\
\hline Duration & $\mathbf{N}$ & \# Firms & Median & Average & Std Dev & $25 \%$ & $75 \%$ & Average & Average \\
\hline All & 7865 & 9 & 5502 & 70634 & 271816 & 34 & 35373 & 0.0041 & 20 \\
\hline Voltage Sag & 444 & 8 & 0 & 15601 & 69990 & 0 & 2771 & 0.0006 & 3 \\
\hline $1-2 \mathrm{sec}$ & 615 & 1 & 2 & 23097 & 94201 & 0 & 7063 & 0.0010 & 5 \\
\hline $1 \mathrm{~min}$ & 200 & 2 & 1502 & 12944 & 53648 & 500 & 5500 & 0.0011 & 5 \\
\hline $15 \mathrm{~min}$ & 212 & 1 & 0 & 46790 & 420257 & 0 & 3266 & 0.0083 & 29 \\
\hline $20 \mathrm{~min}$ & 225 & 1 & 3370 & 18245 & 62847 & 1000 & 10000 & 0.0016 & 7 \\
\hline $30 \mathrm{~min}$ & 647 & 1 & 9045 & 70238 & 237525 & 107 & 44315 & 0.0024 & 14 \\
\hline $1 \mathrm{hr}$ & 2728 & 2 & 4354 & 59983 & 256054 & 23 & 25488 & 0.0037 & 15 \\
\hline $4 \mathrm{hr}$ & 2097 & 6 & 19331 & 119715 & 348509 & 1776 & 77584 & 0.0067 & 35 \\
\hline $8 \mathrm{hr}$ & 568 & 3 & 16568 & 88224 & 306076 & 200 & 69197 & 0.0100 & 45 \\
\hline $12 \mathrm{hr}$ & 129 & 2 & 20746 & 58562 & 126745 & 4320 & 53996 & 0.0187 & \\
\hline
\end{tabular}

Table 3-3 provides a similar summary of the average outage cost for 4 other outage attributes or customer characteristics including season, weekday/weekend, region, and SIC code. The results are shown only for scenarios where the duration was 1 hour.

Table 3-3. Summary of Outage Costs for Key Attributes for a 1-Hour Outage

\begin{tabular}{|c|c|c|c|c|c|c|c|c|c|}
\hline \multicolumn{10}{|c|}{1 HOUR DURATION } \\
\hline & \multicolumn{7}{|c|}{ Total Cost/Event } & \multirow[t]{2}{*}{$\begin{array}{c}\text { Cost Per } \\
\text { Annual } \\
\text { kWh } \\
\text { Usage } \\
\text { Average }\end{array}$} & \multirow{2}{*}{$\begin{array}{c}\text { Cost Per } \\
\text { Peak kW } \\
\text { Average }\end{array}$} \\
\hline SEASON & $\mathbf{N}$ & \# Firms & Median & Average & Std Dev & $25 \%$ & $75 \%$ & & \\
\hline All & 2315 & 7 & 6034 & 67649 & 275911 & 2 & 30269 & 0.0036 & 17 \\
\hline Winter & 421 & 2 & 0 & 39981 & 274447 & 0 & 13062 & 0.0071 & 25 \\
\hline Summer & 1894 & 6 & 8457 & 73799 & 275931 & 224 & 37638 & 0.0034 & 16 \\
\hline \multicolumn{10}{|l|}{ DAY } \\
\hline All & 2728 & 8 & 4354 & 59983 & 256054 & 23 & 25488 & 0.0037 & 15 \\
\hline Weekday & 2491 & 8 & 6034 & 65307 & 267319 & 65 & 29574 & 0.0037 & 17 \\
\hline Weekend & 237 & 3 & 216 & 4028 & 13810 & 0 & 2160 & 0.0015 & NA \\
\hline \multicolumn{10}{|l|}{ REGION } \\
\hline All regions & 2728 & 8 & 4354 & 59983 & 256054 & 23 & 25488 & 0.0037 & 15 \\
\hline Northwest & 834 & 2 & 687 & 28609 & 200482 & 0 & 10160 & 0.0066 & 18 \\
\hline Southwest & 190 & 1 & 1392 & 51909 & 171755 & 106 & 18829 & 0.0039 & 22 \\
\hline Southeast & 1352 & 3 & 11320 & 86477 & 310234 & 68 & 50816 & 0.0033 & 15 \\
\hline West & 120 & 1 & 5617 & 52735 & 218548 & 434 & 24220 & 0.0073 & 33 \\
\hline Midwest & 232 & 1 & 6000 & 28735 & 93286 & 1759 & 19453 & 0.0025 & 11 \\
\hline \multicolumn{10}{|l|}{ SIC } \\
\hline All SIC & 535 & 3 & 18242 & 100148 & 358231 & 940 & 61348 & 0.0034 & 20 \\
\hline Agriculture & 4 & 2 & 2074 & 8476 & 14247 & 499 & 16452 & 0.0002 & 1 \\
\hline Mining & 17 & 2 & 52456 & 76463 & 87720 & 6459 & 93993 & 0.0019 & 11 \\
\hline Construction & 1 & 1 & 466 & 466 & & 466 & 466 & 0.0000 & 0 \\
\hline Manufacturing & 331 & 2 & 24828 & 131214 & 426764 & 8528 & 94911 & 0.0035 & 21 \\
\hline Telco \& Utilities & 46 & 2 & 1489 & 64339 & 239499 & 52 & 17620 & 0.0031 & 18 \\
\hline Trade \& Retail & 19 & 2 & 7030 & 11153 & 12589 & 522 & 17205 & 0.0024 & 13 \\
\hline Finance, Ins., R.E. & 11 & 2 & 4746 & 203540 & 624425 & 0 & 27785 & 0.0415 & 168 \\
\hline Services & 84 & 2 & 761 & 18043 & 41168 & 0 & 17864 & 0.0020 & 9 \\
\hline Public Admin & 22 & 2 & 9938 & 85779 & 139384 & 16 & 134796 & 0.0039 & 19 \\
\hline
\end{tabular}


The data suggest that outage costs on a per event basis are higher in the summer than the winter ( $\$ 73,799$ versus $\$ 39,981)$; are higher on weekdays than weekends $(\$ 65,307$ versus $\$ 4,028)$; are higher in the Southeast $(\$ 86,477$ per event) than in the Northwest $(\$ 28,609$ per event) or Midwest (\$28,735 per event); and are higher for finance /insurance/real estate $(\$ 105,468$ per event) and manufacturing $(\$ 88,483)$ than other business and government sectors.

These patterns generally hold for outage cost estimates on a per annual $\mathrm{kWh}$ and per $\mathrm{kW}$ basis with a few modifications. First, in terms of season, the outage cost per event is substantially higher in the summer than the winter, but this difference is reversed when the amount of usage or demand is taken into account. The outage cost per annual $\mathrm{kWh}$ is $\$ .0071$ for winter outages and it is $\$ .0034$ for summer outages. Similarly, the outage cost per $\mathrm{kW}$ of demand is $\$ 25$ for winter and $\$ 16$ for summer. This suggests that seasonal differences in outage costs are closely tied to consumption.

The day of the week data show that outage costs on a per annual kWh are much higher during the week than on the weekend for large commercial and industrial customers. Weekday outage costs per annual $\mathrm{kWh}$ are $\$ .0037$ for weekdays and $\$ .0015$ for weekends. Data on outage costs per $\mathrm{kW}$ were not available for the weekend scenario. These differences are suggestive of much lower average outage costs during periods when most businesses are closed (weekends) compared to when they are open (weekdays).

For data on regions, the rank order of the regions is somewhat different when the outage costs are measured on a per annual $\mathrm{kWh}$ and $\mathrm{kW}$ basis. The West region has the highest costs per annual kWh (\$.0066) and cost per kW (\$33), while the Midwest (at \$.0025 per annual kWh and $\$ 11$ per $\mathrm{kW}$ ) has the lowest values.

Finally, in terms of SIC codes, finance/insurance/real estate has the highest cost per event, and also has the highest costs per annual $\mathrm{kWh}$ costs $(\$ 0.0252)$ and per $\mathrm{kW}$ costs $(\$ 90)$. The remaining business types range from $\$ 0.0010$ to $\$ 0.0057$ on a per annual $\mathrm{kWh}$ basis with trade/retail being the highest and construction being the lowest.

The two most problematic scenario characteristics are whether the firm has a back-up system, and whether the scenario includes receipt of advance warning. The only way to make the cost comparisons meaningful is to be certain that one is comparing the same scenarios while varying the characteristics, and do so with essentially the same respondents. For advanced warning, only one study provided this direct comparison, such that for a 1-hour summer weekday outage, the event cost with advance warning was $\$ 302,206$, and without advance warning it was $\$ 475,680$ $(n=205)$, or more than 50\% higher. Back-up seems more promising: whether one has back-up is a standard question, yet there was approximately $20 \%$ item non-response for the meta-set. Nevertheless, for two scenarios - 1-hour summer weekday and a 4-hour summer weekday, we have adequate data to report:

\begin{tabular}{|c|r|r|r|c|}
\hline Outage Scenario & Has Back-up & \multicolumn{1}{c|}{ N } & No Back-up & N \\
\hline 1-Hour Summer Weekday Outage & $\$ 215,012$ & 1,042 & $\$ 1,052,564$ & 676 \\
\hline 4-Hour Summer Weekday Outage & $\$ 307,272$ & 873 & $\$ 1,175,910$ & 663 \\
\hline
\end{tabular}


In the regression models for the Customer Damage Function shown in the next section, these two variables were found to be unusable due to the data structure inconsistencies. 


\subsection{Customer Damage Function Estimation}

The summary of outage costs for the key characteristics outlined above provides a measure of whether the combination of various studies fit intuitively with expectations of outage costs for this sector. However, the results may not be particularly useful when examined one variable at a time. The average value of the outage costs for any given descriptor variable is a function of the outage attributes, region, and the customer types that answered that particular scenario. As noted at the beginning of this section, the combination of customer and outage characteristics can vary substantially depending on the variables being examined. To adequately control for these varying influences, a multivariate analysis was conducted to develop a multivariate customer damage function. In this manner, the information about the scenario and firm characteristics was used to estimate a general customer damage function expressing commercial and industrial customers' outage costs as a function of outage duration, onset time, season, and various customer characteristics such as annual usage, number of employees and other variables.

The ideal conceptual framework within which to analyze the above-described data is statistical regression. However, the use of an Ordinary-Least Squares (OLS) approach is inappropriate for typical outage cost data. The key issue is the usual response distribution for the dependent variable - outage costs. In almost all studies, and including the large commercial and industrial customers, a significant number of respondents will report "0" (zero) outage costs for many scenarios. This is particularly true of short duration outages, but may be true of even longer ones at certain times of the day or seasons. As a result, standard OLS regression techniques will yield biased parameter estimates. To overcome this problem, the analysis reported below uses Tobit regression $^{3}$. Tobit is a useful technique when the underlying distribution of the dependent variable is censored or truncated in some fashion. It essentially combines the estimation of the probability that the customers" outage cost is " 0 " and the estimation of the value of the outage cost if it is non-zero. This maximum likelihood regression procedure estimates the regression parameters under the assumption that all non-positive measurements are truncated at zero (i.e., a customer cannot have a negative outage cost).

In conducting the analysis, a second set of techniques was used to improve the estimation process. The typical distribution of outage costs in addition to having a large number of zeros will also often have very extreme values. A few values in the positive tail of the distribution may be as much as 100 or 1000 times higher than other values. In most outage cost studies using surveys (including those used in this meta-analysis), a great deal of attention is given to checking to confirm high values or excluding them if they appear to be inappropriate (due, for example, to calculation error or misunderstanding of the question). Since for the project the original surveys are not available, two procedures were used to handle the potential outliers in the distribution. First, the top $0.05 \%$ of the entire calculated outage cost distribution was truncated to eliminate extremely high values.

Second, the analysis was conducted on the logarithm of the outage cost rather than the actual outage cost for the commercial and industrial customers. The decision to use a lognormal distribution was based on several considerations. Using a lognormal transformation gives the

\footnotetext{
${ }^{3}$ See Chapter 7, "Limited Outcomes: The Tobit Model" in Regression Models for Categorical and Limited Dependent Variables, J.S. Long, Sage Publications: Thousand Oaks, CA, 1997.
} 
underlying distribution of outage costs a more normal shape with less severe tails (Figures 3-1, 3-2, 3-3). This transformation helps assure that the distribution of the error term is normal (an important assumption in using regression) as any skew in the error term is likely associated with the extreme values of some outage costs. Finally, the logarithm produces a distribution in which a customers' outage cost is " 0 " when the duration is " 0 ", but allows for a rapid nonlinear rise in outage costs for even a momentary event.

Because of this log transformation, the metric values of the parameters in a Tobit model cannot be directly interpreted in terms of interruption costs. However, exponentiation of the function produces a predicted outage cost given values of variables in the models. To observe the magnitude of the impact of the variables in the models on the interruption cost it is therefore necessary to compare the predictions made by the function under varying assumptions. For example, it is possible to observe the effects of duration on outage cost holding the other variables constant at their sample means. In this way, a prediction is obtained for customer outage costs under different outage conditions.

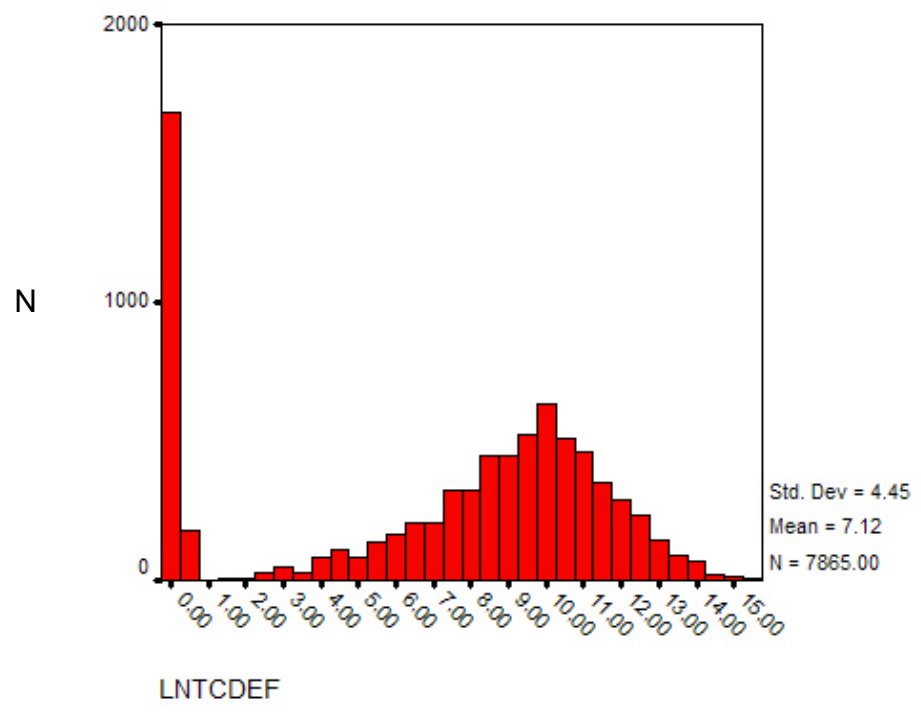

Figure 3-1. Logged Per Event Cost Distribution 


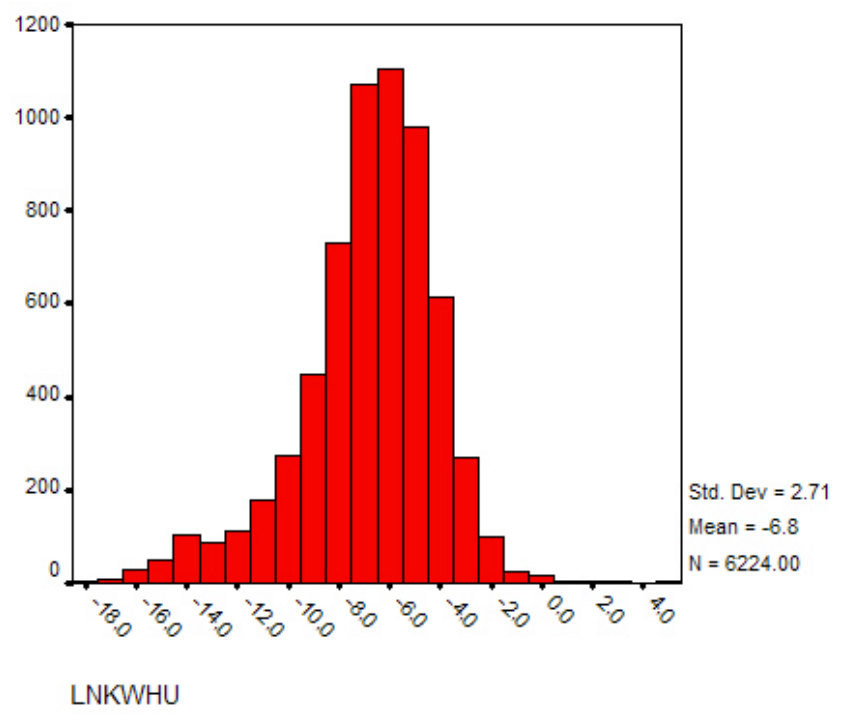

Figure 3-2. Logged Outage Cost per Annual kWh

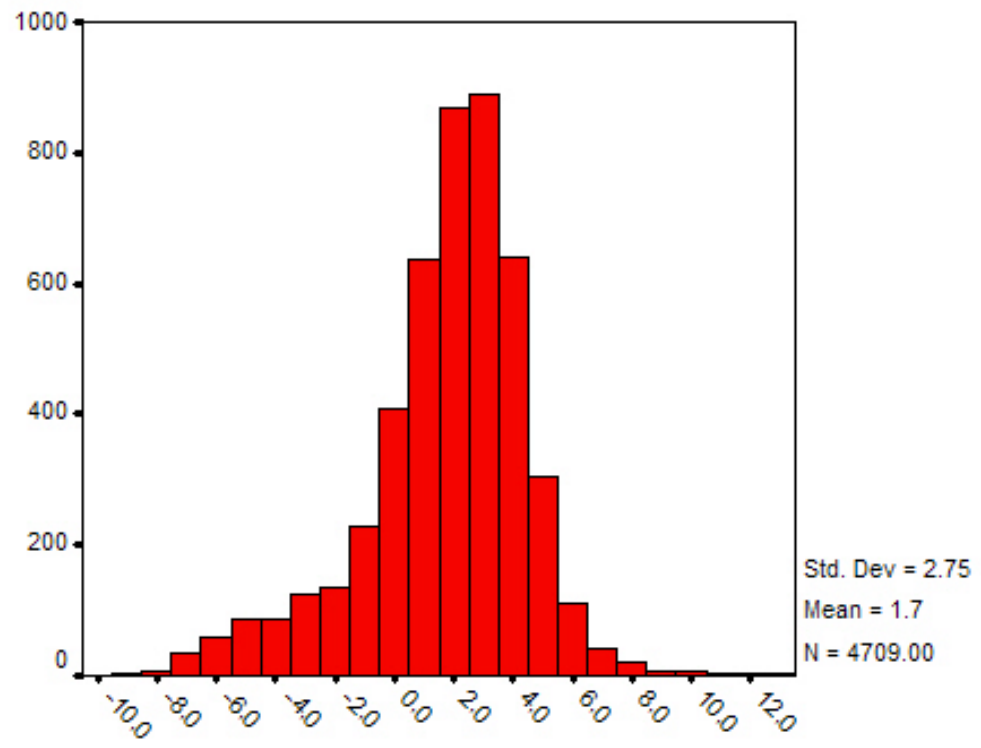

LNKWHD

Figure 3-3. Logged Outage Cost per Peak kW

To develop a set of models, several combinations of the variables representing attributes of the outage (e.g., duration, time of day, advanced warning) and customer characteristics (e.g., number of employees, SIC code, and presence of backup) were tested. Because not all studies included the same variables, there was significant data loss when models using some variables were estimated. For example, very few studies included scenarios with advance warning as an attribute so if this attribute was included in the Tobit model the number of cases was substantially reduced. In the end, the regression models for the large C\&I (and the other two 
customer groups) consisted of variables that were significant, were not collinear (as is region with season, since the Northwest utilities included winter scenarios but none of the Southeast regions did), and did not exclude a substantial number of cases (for example, with advanced warning, since very few scenarios included that variable).

Table 3-4 below describes two Tobit regression models that specify the relationship between various outage and customer characteristics and customer outage cost for which sufficient data from multiple studies were available. Model One contains only the parameters associated with outage attributes and customer size. The largest number of cases is available to estimate this model. Model Two includes parameters describing the business activities being interrupted. Information on the customers SIC code is available for a smaller subset of the data.

These customer damage functions are a key output from this research. The models can be used to estimate outage costs for a wide range of outages with different attributes (e.g., duration, time of day) and for different types of customers (e.g., large versus small companies). They replace the enormous number of tables that would be required to summarize all the different combinations of characteristics. Using this information is relatively straight-forward. To simulate the outage cost for a particular set of outage or customer characteristics one multiplies the appropriate value for each variable times the coefficient for that variable. The multiplications are summed across the variables and added to the constant (first entry for each model). Since the variable being predicted - i.e., outage cost - has been transformed to be the log of the outage cost, as a final step in the simulation the antilog of the summed value must be taken. The resulting value is the predicted outage cost for the set of values used for each independent variable. 
Table 3-4. Tobit Regression Models for Predicting Outage Costs

\begin{tabular}{|c|c|c|c|c|c|c|}
\hline \multirow[b]{2}{*}{ Predictor } & \multicolumn{3}{|c|}{ Model One } & \multicolumn{3}{|c|}{ Model Two } \\
\hline & Parameter & S.E. & Pr $>$ ChiSq & Parameter & S.E. & $\operatorname{Pr}>$ ChiSq \\
\hline Intercept & 7.7954 & 0.1377 & $<.0001$ & 7.6941 & 0.1542 & $<.0001$ \\
\hline Duration (hours) & 0.5753 & 0.0376 & $<.0001$ & 0.5771 & 0.0357 & $<.0001$ \\
\hline Duration Squared & -0.0338 & 0.0035 & $<.0001$ & -0.0331 & 0.0032 & $<.0001$ \\
\hline Number of Employees & 0.0007 & 0.0001 & $<.0001$ & 0.0006 & 0.0001 & $<.0001$ \\
\hline Annual kWh & $2.52 \mathrm{E}-08$ & 0.004 & $<.0001$ & $2.25 \mathrm{E}-08$ & 0.0036 & $<.0001$ \\
\hline Interaction Duration and $\mathrm{kWh}$ & $-1.80 \mathrm{E}-09$ & 0.001 & 0.0703 & $-1.30 \mathrm{E}-09$ & 0.0009 & 0.1282 \\
\hline Morning & -0.5624 & 0.1308 & $<.0001$ & -0.4319 & 0.1144 & 0.0002 \\
\hline Night & -1.3857 & 0.1841 & $<.0001$ & -1.4464 & 0.1739 & $<.0001$ \\
\hline Weekend & -0.7149 & 0.1485 & $<.0001$ & -0.6482 & 0.1441 & $<.0001$ \\
\hline Winter & 0.8992 & 0.0996 & $<.0001$ & 0.8376 & 0.0901 & $<.0001$ \\
\hline Manufacturing & & & & 0.5292 & 0.1166 & $<.0001$ \\
\hline Mining & & & & 1.1378 & 0.2484 & $<.0001$ \\
\hline Construction & & & & 0.9168 & 0.808 & 0.2565 \\
\hline Transportation/Utilities & & & & -0.193 & 0.1585 & 0.2233 \\
\hline Finance/Insurance/Real Est. & & & & 0.3252 & 0.2841 & 0.2522 \\
\hline Services & & & & -0.4661 & 0.1363 & 0.0006 \\
\hline Public & & & & 0.0253 & 0.2431 & 0.917 \\
\hline Number of Observations & & 3198 & & & 2542 & \\
\hline Zero Response & & 718 & & & 427 & \\
\hline Log Likelihood & & -6904 & & & -5087 & \\
\hline
\end{tabular}

Figure 3-4, below, displays a comparison of the results of the customer damage function in Model One over the durations found in the sample dataset under varying times of day and seasons. This model controls for the customers size in terms of number of employees and annual consumption $(\mathrm{kWh})$. The predicted results assume the average value for number of employees and annual $\mathrm{kWh}$.

It is evident that the relationship between outage costs or damages and duration is non-linear increasing slowly within the first hour, accelerating through the second through the eighth hours, and declining thereafter. All of the predictions are positive at the intercept representing the impact of momentary interruptions. Outage costs for winter outages are significantly higher when controlling for customer size and consumption than those experienced in summer; and outage costs during the night and on weekends are significantly lower as expected. 


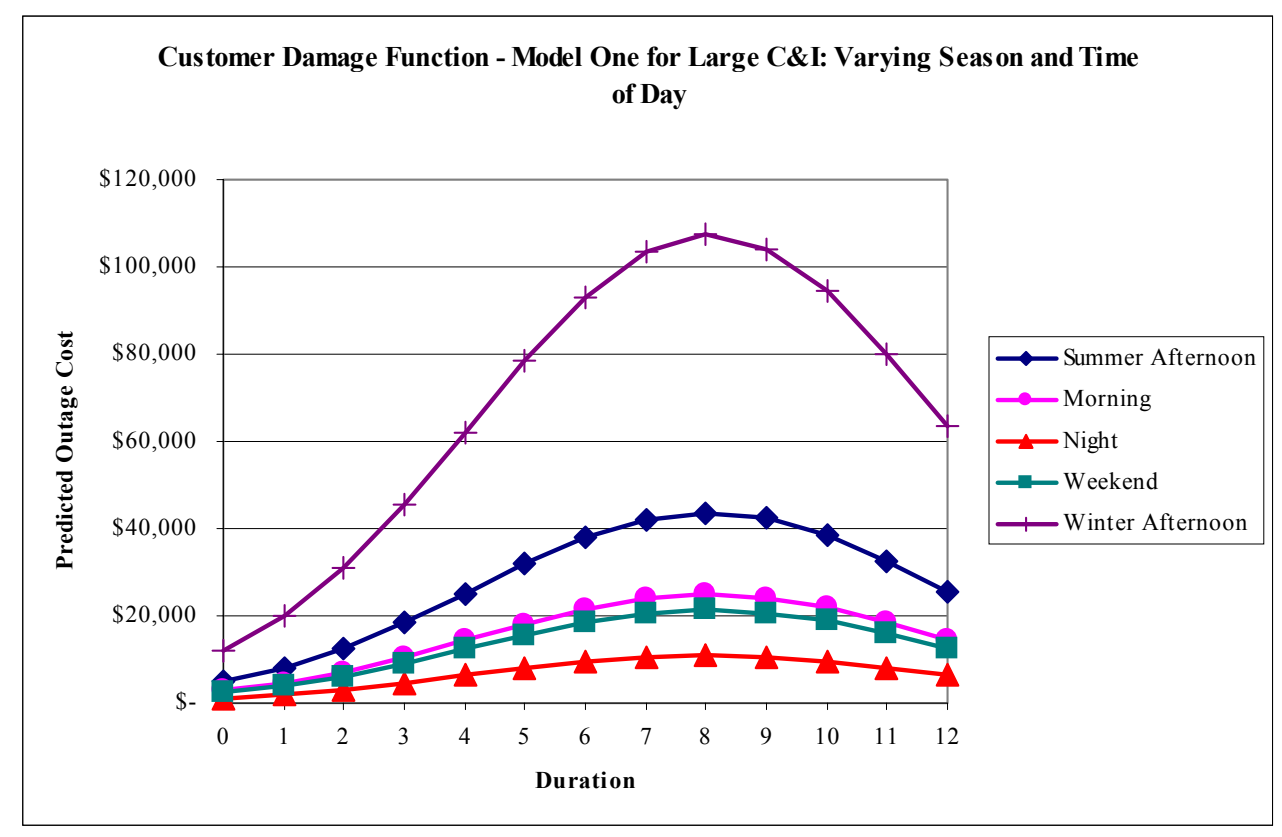

Figure 3-4. Customer Damage Functions - Model One Varying Season and Time of Day

The results show that, for the large commercial and industrial customer pool, an average customer with 373 employees and 17.5 million annual $\mathrm{kWh}$ consumption will experience approximately $\$ 20,000$ in costs from a 1-hour afternoon outage in the winter and $\$ 8,166$ in costs for a summer afternoon 1-hour outage. These costs increase sharply as duration increases in the winter; costs also increase substantially with duration in the summer.

The curvilinear nature of the line suggests that for large commercial and industrial establishments costs actually moderate with longer outages. This makes sense as focus groups and interview respondents often note that at some point employees are sent home, shifts are eliminated, and the outages extend into hours that would be normally non-productive (evening and night time hours). Since none of the studies measure costs beyond 12 hours, it is impossible to say from this data when and by how much costs rise as an outage extends into multiple days.

It is also possible to estimate the customer damage function by varying other parameters. For example, it is possible to observe the effect of customer size (measured in $\mathrm{kWh}$ or employees), by holding other parameters constant and systematically varying the size of customer. Figure 35 , below, displays the results of estimating customer outage cost for summer afternoons varying annual $\mathrm{kWh}$ consumption away from the mean by a factor of 10 (i.e., from 1,750,970 kWh to 175,097,016). 


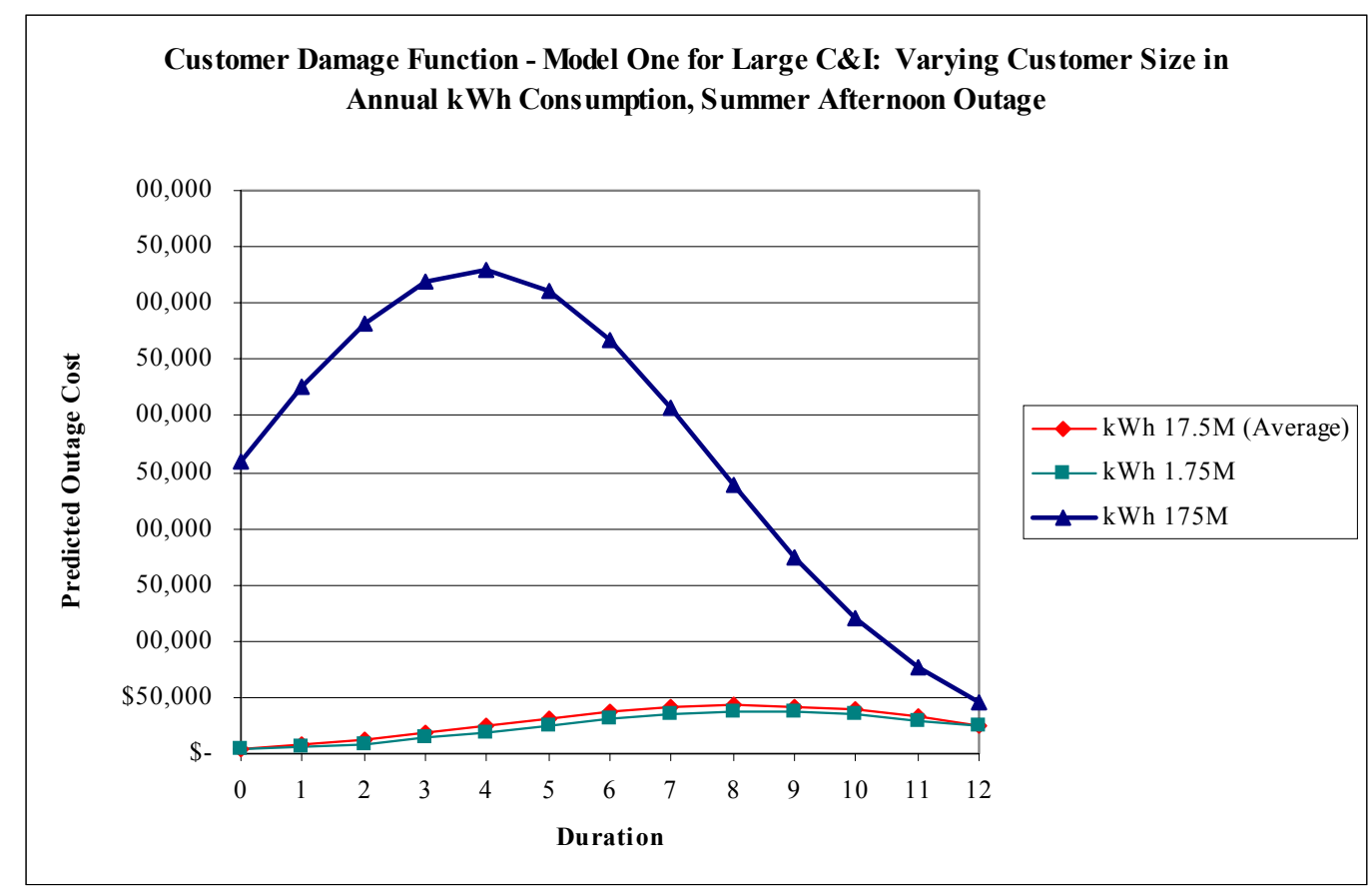

Figure 3-5. Customer Damage Functions - Model One Varying Customer Size

It is evident that the size of a customer's load dramatically increases the outage cost, and the relationship is not linear. A decrease of 90 percent in $\mathrm{kWh}$ consumption from the average consumption in the sample results in only a small reduction in customer outage cost, while increasing it by a factor of 10 dramatically increases customer interruption cost. In addition, the model results show that for larger customers, the costs start high and escalate rapidly during the first three to four hours. But if an afternoon outage lasts beyond 5 to 6 hours, the costs come down dramatically as facilities start to take actions to lower their costs.

In addition to outage attributes and customer size, Model Two contains parameters describing the effects of business type on outage costs. A separate model was estimated for this analysis because several of the surveys included in the study did not report business type. Figure 3-6, below, displays the customer damage functions from Model Two estimated for different types of businesses. All of the other parameters in the model are held at their means thus allowing an assessment of the independent effect of business type. That is, the dark blue line (with diamonds) indicates the customer damage function for outages on a summer afternoon, experienced by establishments involved in retail trade using an average of 17,509,7016 kWh annually and with about 373 employees.

It is apparent in the figure that interruption costs for mining far outstrip those of any other business activity. They are more than double the costs experienced by businesses involved in retail trade and more than four times the cost experienced by customers involved in public sector activities (e.g., schools and municipal buildings). The next highest outage costs are for customers involved in construction. Costs for manufacturing and construction are significantly higher than those of other businesses. 
Costs for other business types are relatively close to those of retail trade, though the differences among them are statistically significant.

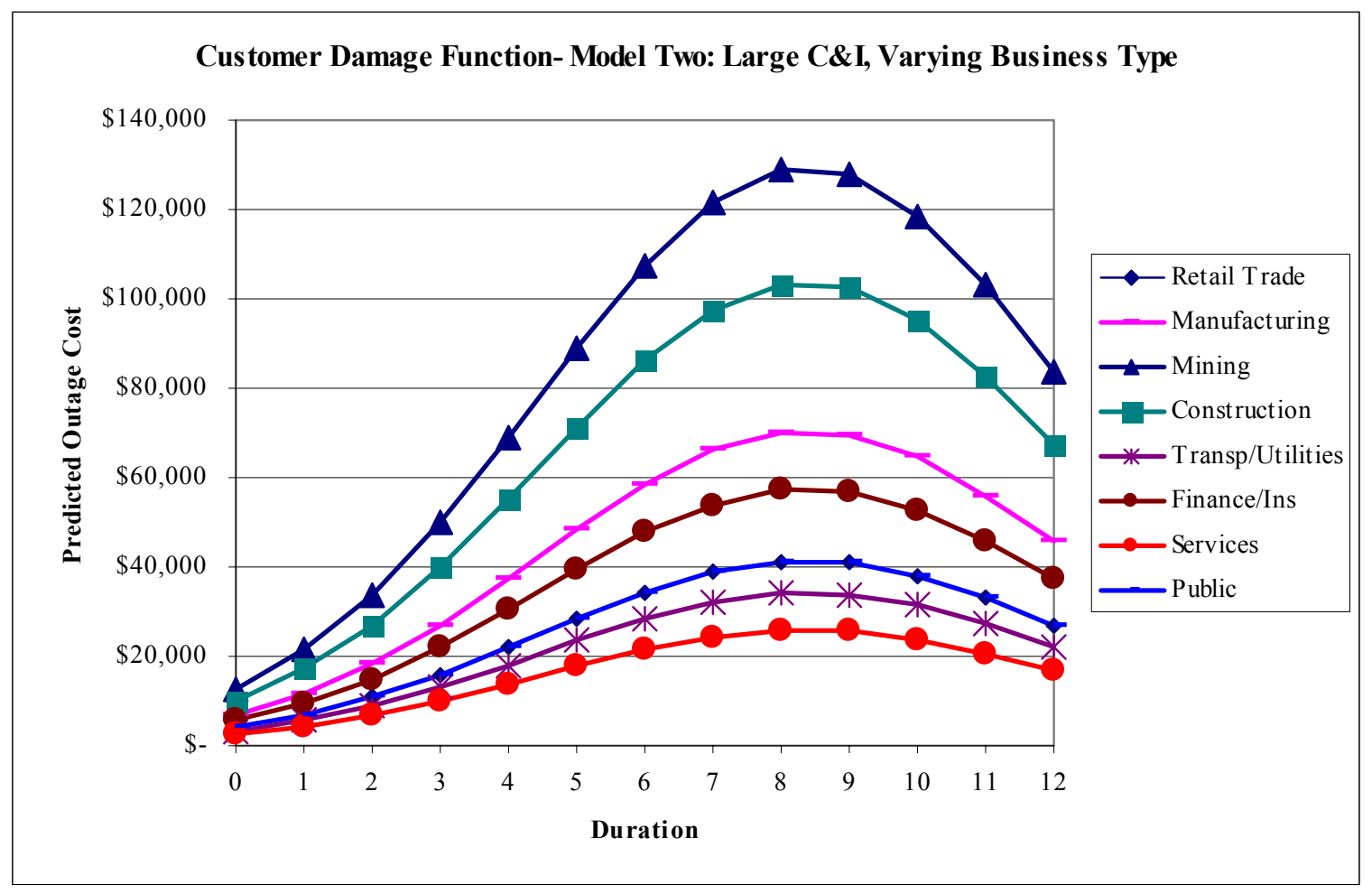

Figure 3-6. Customer Damage Functions For Various Business Types

From the above examples it should be apparent that it is possible to use the customer damage functions from the above models to estimate customer outage costs under a wide variety of conditions. However, it is not appropriate to use these functions to estimate outage costs for individual customers. The regression functions used above are really predicting the mean of the customer outage cost for populations of customers with different characteristics under different conditions. There is substantial unexplained variation among customers in the outage costs they experience resulting from factors that are not accounted for in the above equations (e.g., process design differences, resistance of equipment to electric disturbances, etc.) and will not generally be known without an in-depth interview. The existence of these unknowns virtually guarantees that the prediction for any individual customer from the above functions will be significantly in error. Appendix D examines this issue from the standpoint of estimation of individual customer damages in a legal setting.

The above caution does not apply to the application of the above equations for reasonably large populations of customers where the law of large numbers and central limit theorem ensure that random but significant differences among customers do not produce estimates that deviate dramatically from the predictions made by the above equations. 


\section{Small and Medium Commercial and Industrial Results}

The small-medium commercial and industrial dataset is built from 9 studies conducted by 8 companies and includes approximately 5,200 respondents. Overall, there were approximately 24,000 total responses available for the analysis. The distribution of the available data across various outage attributes, years, and customer characteristics is described first. A summary of the multivariate analysis is presented second.

In terms of coverage, Table 4-1 summarizes the number of records available for analysis by region, season, day of week, and year of study. Overall there were 23,800 responses to various scenario combinations across the studies, considerably more than were available for analysis in the large commercial and industrial customer database. The results show that there are from 400 to several thousand responses depending on the scenario and region combination (with the exceptions of two small studies; one with 7 cases and the other with 24 cases). There are a substantial number of cases available for the analysis of summer and winter scenarios occurring on both weekdays and weekends. The data also vary reasonably across regions although, again, there is no coverage for the Northeast. Most of the studies were completed in the past 5 to 6 years, but two studies date back to the late 1980's and early 1990's. Overall, the data in Table 41 suggest sufficient coverage to develop models of outage costs for a wide cross-section of the country and across a range of scenarios.

Table 4-1. Number of Cases by Region, Company, Season and Day of Week - SmallMedium C\&I

\begin{tabular}{|c|c|c|c|c|c|c|c|c|c|c|}
\hline \multirow[b]{2}{*}{ Region - Company } & \multirow[b]{2}{*}{ Season } & \multirow[b]{2}{*}{ Day of Week } & \multicolumn{8}{|c|}{ Year of Survey } \\
\hline & & & 1989 & 1991 & 1993 & 1997 & 1999 & 2000 & 2002 & Total \\
\hline Northwest-1 & Winter & Weekday & 1650 & & & & & & & 1650 \\
\hline \multirow[t]{2}{*}{ Northwest-2 } & Summer & Weekend & & & & & 877 & & & 877 \\
\hline & Summer & Weekday & & & & & 4467 & & & 4467 \\
\hline \multirow[t]{3}{*}{ West } & Summer & Weekday & & & & & & 2105 & & 2105 \\
\hline & Winter & Weekend & & & & & & 860 & & 860 \\
\hline & Winter & Weekend & & & & & & 443 & & 443 \\
\hline Midwest & Summer & Weekday & & & & & & & 2014 & 2014 \\
\hline \multirow[t]{3}{*}{ Southwest } & Summer & Weekend & & & & & & 515 & & 515 \\
\hline & Summer & Weekday & & & & & & 3767 & & 3767 \\
\hline & Winter & Weekday & & & & & & 1006 & & 1006 \\
\hline Southeast-1 & Summer & Weekday & & & & 7 & & & & 7 \\
\hline \multirow[t]{2}{*}{ Southeast-2 } & Summer & Weekend & & & & 935 & & & & 935 \\
\hline & Summer & Weekend & & & 24 & 3,805 & & & & 3829 \\
\hline Southeast-3 & Summer & Weekday & & 1325 & & & & & & 1325 \\
\hline \multicolumn{3}{|l|}{\begin{tabular}{|l|} 
TOTAL \\
\end{tabular}} & 1650 & 1325 & 24 & 4747 & 5344 & 8696 & 2014 & 23800 \\
\hline
\end{tabular}

While the data in Table 4-1 show fairly broad geographical coverage and coverage across types of outages, they also indicate the need for caution in interpreting the data for certain combinations of characteristics, just as was true with the large commercial and industrial data. For example, all of the 1989 data are winter weekday scenarios from one region (the Northwest), while all of the 1991 data are summer weekdays from the Southeast. Comparing the average outage costs for the years 1989 and 1991 without some effort to control for the effects of the differences in region and type of scenario would be misleading. The next few tables provide a 
summary of the observed outage costs for a few key variables but, again, caution must be used in interpreting the results because of these coverage issues.

Table 4-2 shows the distribution of outage costs (per event, per annual $\mathrm{kWh}$, and per $\mathrm{kW}$ ) across by outage duration. The results show outage costs rising from an average of $\$ 203$ for a voltage sag to $\$ 7,361$ for an 8-hour outage. The results trend generally upward as would be expected. There are two deviations from this trend. First, the 1-2 second outage has a significantly higher per event cost $(\$ 1,230)$ than the events on either side of the duration curve (voltage sag at \$203 and 1-minute outage at \$543). The second deviation is the downward trend in outage cost from the 8 -hour duration $(\$ 7,361$ per event) to the 12 -hour duration $(\$ 5,590$ per event). It is possible that these differences represent a methodological artifact as only one study used the 1-2 second duration and the 12-hour duration. A discussion of the effect of duration on outage costs in the context of a multivariate model controlling for differences among the studies is provided later in this section.

Table 4-2. Costs by Duration - Small-Medium C\&I

\begin{tabular}{|c|c|c|c|c|c|c|c|c|c|}
\hline \multicolumn{10}{|c|}{ BY DURATION } \\
\hline & \multicolumn{7}{|c|}{ Total Cost/Event } & \begin{tabular}{|c|} 
Cost Per Annual \\
kWh Usage
\end{tabular} & $\begin{array}{l}\text { Cost Per } \\
\text { Peak kW } \\
\end{array}$ \\
\hline Duration & $\mathbf{N}$ & \# Firms & Median & Average & Std Dev & $25 \%$ & $75 \%$ & Average & Average \\
\hline All & 23800 & 9 & 209 & 2735 & 8608 & 0 & 1512 & 0.0218 & 55 \\
\hline Voltage Sag & 882 & 8 & 0 & 203 & 3200 & 0 & 0 & 0.0015 & 1 \\
\hline $1-2 \mathrm{sec}$ & 2072 & 1 & 3 & 1230 & 5153 & 0 & 439 & 0.0132 & 34 \\
\hline $1 \mathrm{~min}$ & 255 & 2 & 4 & 543 & 2078 & 1 & 100 & 0.0028 & 2 \\
\hline $15 \mathrm{~min}$ & 413 & 1 & 0 & 831 & 3983 & 0 & 189 & 0.0040 & 9 \\
\hline $20 \mathrm{~min}$ & 353 & 1 & 100 & 980 & 4901 & 5 & 500 & 0.0051 & 3 \\
\hline $30 \mathrm{~min}$ & 980 & 1 & 365 & 2367 & 7020 & 35 & 1599 & 0.0211 & 49 \\
\hline $1 \mathrm{hr}$ & 10849 & 2 & 108 & 1859 & 6623 & 0 & 940 & 0.0155 & 40 \\
\hline $4 \mathrm{hr}$ & 5836 & 6 & 756 & 4220 & 10634 & 13 & 3135 & 0.0368 & 91 \\
\hline $8 \mathrm{hr}$ & 1319 & 3 & 1250 & 7361 & 15653 & 1 & 6685 & 0.0431 & 99 \\
\hline $12 \mathrm{hr}$ & 841 & 2 & 1620 & 5590 & 11230 & 540 & 5400 & 0.0408 & \\
\hline
\end{tabular}

Table 4-2 also shows the outage costs converted to a cost per annual $\mathrm{kWh}$ and per $\mathrm{kW}$ basis. On a per annual $\mathrm{kWh}$ basis, the results also suggest that outage costs generally increase with duration from $\$ 0.0015$ to $\$ 0.0431$ across the same range of duration from a voltage sag to an 8 hour outage (with the same deviations). The data on outage cost per $\mathrm{kW}$ of demand is less straightforward. The costs range from $\$ 1$ per $\mathrm{kW}$ of demand for a voltage sag to $\$ 99$ per $\mathrm{kW}$ for an 8-hour outage. $\mathrm{T}$ he costs also increase in a stepwise fashion with large jumps in the average cost per $\mathrm{kW}$ between 20 minutes and 30 minutes and between 1 hour and 4 hours. The jump between 20 and 30 minutes is likely an artifact of the underlying study that used these two durations. The jump between 1 hour and 4 hours is more robust as several studies included these two durations among their scenarios.

Table 4-3 provides a summary of the average outage cost for 4 other outage attributes or customer characteristics including season, weekday/weekend, region, and SIC code. The results are shown only for scenarios where the duration is 1 hour. The data suggest that outage costs on a per event basis are higher in the winter than the summer $(\$ 2,643$ versus $\$ 1,897)$; are higher on weekdays than weekends (\$2158 versus $\$ 768$ ); are higher in the West than in other regions of the country; and are higher for manufacturing and agriculture. 
Table 4-3. Summary of Outage Costs for Key Attributes for a 1-Hour Outage

\begin{tabular}{|c|c|c|c|c|c|c|c|c|c|}
\hline \multicolumn{10}{|c|}{1 HOUR DURATION } \\
\hline & \multicolumn{7}{|c|}{ Total Cost/Event } & $\begin{array}{c}\text { Cost Per Annual } \\
\text { kWh Usage }\end{array}$ & $\begin{array}{l}\text { Cost Per } \\
\text { Peak kW }\end{array}$ \\
\hline REGION & $\mathbf{N}$ & \# Firms & Median & Average & Std Dev & $25 \%$ & $75 \%$ & Average & Average \\
\hline All regions & 10849 & 8 & 108 & 1859 & 6623 & 0 & 940 & 0.0155 & 40 \\
\hline Northwest & 3596 & 2 & 54 & 1686 & 6575 & 0 & 643 & 0.0111 & 18 \\
\hline Southwest & 3064 & 1 & 305 & 2176 & 6845 & 47 & 1275 & 0.0282 & 66 \\
\hline Southeast & 3363 & 3 & 0 & 1484 & 5721 & 0 & 561 & 0.0133 & 26 \\
\hline West & 411 & 1 & 1045 & 4581 & 11194 & 157 & 4180 & 0.0448 & 102 \\
\hline Midwest & 415 & 1 & 200 & 1369 & 5270 & 25 & 1000 & 0.0072 & 4 \\
\hline \multicolumn{10}{|l|}{\begin{tabular}{|l} 
SEASON \\
\end{tabular}} \\
\hline All & 8080 & 7 & 117 & 2066 & 7135 & 0 & 1045 & 0.0181 & 44 \\
\hline Winter & 1833 & 3 & 146 & 2643 & 8699 & 0 & 1262 & 0.0196 & 47 \\
\hline Summer & 6247 & 7 & 112 & 1897 & 6597 & 0 & 1009 & 0.0175 & 46 \\
\hline \multicolumn{10}{|l|}{ DAY } \\
\hline All & 10849 & 8 & 108 & 1859 & 6623 & 0 & 940 & 0.0155 & 40 \\
\hline Weekday & 8522 & 8 & 157 & 2158 & 7200 & 0 & 1125 & 0.0176 & 44 \\
\hline Weekend & 2327 & 3 & 5 & 768 & 3626 & 0 & 336 & 0.0068 & 25 \\
\hline \multicolumn{10}{|l|}{ SIC } \\
\hline All SIC & 4278 & 3 & 108 & 2183 & 7478 & 0 & 1080 & 0.0147 & 29 \\
\hline Agriculture & 176 & 2 & 22 & 2023 & 8897 & 0 & 529 & 0.0190 & 66 \\
\hline Mining & 16 & 1 & 22 & 898 & 1632 & 0 & 940 & 0.0032 & 4 \\
\hline Construction & 132 & 2 & 212 & 2221 & 4121 & 0 & 2208 & 0.0229 & 47 \\
\hline Manufacturing & 559 & 2 & 1080 & 5040 & 10627 & 0 & 4860 & 0.0236 & 52 \\
\hline Telco \& Utilities & 237 & 2 & 108 & 1786 & 5502 & 0 & 1045 & 0.0100 & 23 \\
\hline Trade \& Retail & 1382 & 2 & 209 & 1927 & 7210 & 0 & 994 & 0.0123 & 35 \\
\hline Finance, Ins., R.E. & 189 & 2 & 43 & 2072 & 7174 & 0 & 661 & 0.0276 & 53 \\
\hline Services & 1440 & 2 & 35 & 1504 & 6619 & 0 & 561 & 0.0116 & 15 \\
\hline Public Admin & 147 & 1 & 1 & 1442 & 4471 & 0 & 540 & 0.0094 & 20 \\
\hline
\end{tabular}

These patterns generally hold for outage cost estimates on a per annual $\mathrm{kWh}$ and per $\mathrm{kW}$ basis with a few modifications. First, in terms of season, the outage cost per event is substantially higher in the winter than the summer, but this difference is reduced when the amount of usage or demand is taken into account. The outage cost per annual $\mathrm{kWh}$ is $\$ 0.0196$ for winter outages and $\$ 0.0175$ for summer outages. Similarly, the outage cost per $\mathrm{kW}$ of demand is $\$ 47$ for winter and $\$ 46$ for summer. This suggests that seasonal differences in outage costs are closely tied to consumption.

In contrast, the day of the week data show that outage costs on a per annual $\mathrm{kWh}$ and per $\mathrm{kW}$ basis are much higher during the week than on the weekend for small commercial and industrial customers. Weekday outage costs per annual $\mathrm{kWh}$ are $\$ 0.0176$ for weekdays and $\$ 0.0068$ for weekends, while costs per $\mathrm{kW}$ are $\$ 44$ for weekdays but only $\$ 25$ for weekends. These differences illustrate the much lower average outage costs during periods when most businesses are closed (weekends) compared to when they are open (weekdays).

For data on regions, the results by $\mathrm{kWh}$ and $\mathrm{kW}$ largely mirror the cost per event data. The West region has the highest costs per annual $\mathrm{kWh}(\$ 0.0448)$ and cost per $\mathrm{kW}(\$ 102)$, while the Midwest (at $\$ 0.0072$ per annual $\mathrm{kWh}$ and $\$ 4$ per $\mathrm{kW}$ ) and the Northwest (at $\$ 0.0111$ per annual $\mathrm{kWh}$ and $\$ 18$ per $\mathrm{kW}$ ) have the lowest values. 
In terms of SIC codes, the data on a per annual $\mathrm{kWh}$ and per $\mathrm{kW}$ basis are fairly similar to the per event data with two exceptions. Manufacturing, which has the highest cost on a per event basis, also has high costs on a per annual kWh (\$0.0236) and per $\mathrm{kW}(\$ 52)$ basis. Mining, telecommunications/utilities, trade and retail, services, and public administration all tend to have low costs per event as well as low costs per annual $\mathrm{kWh}$ and $\mathrm{kW}$ relative to other SIC groups. The two key exceptions are agriculture and finance/insurance/real estate. Agriculture has a low per event cost and a fairly low per annual $\mathrm{kWh}$ cost $(\$ 0.019)$, but a relatively high per $\mathrm{kW}$ cost (\$66). Finance/insurance/real estate has a relatively low cost per event but has relatively high per annual $\mathrm{kWh}$ costs $(\$ 0.0276)$ and per $\mathrm{kW}$ costs $(\$ 53)$

Finally, as with the large C\&I data, there was only one study where receipt of advance warning was examined in a noticeable way, and the results there were still counter-intuitive, with receipt of advance warning being associated with higher outage costs than those who received no warning. This anomaly may be a result of the manner in which the question was asked, in that those without advance warning received a different set of possible numeric values (for a 'willingness to pay' measure) than those whose scenario included an advance warning. Back-up numbers were particularly counterintuitive. As we shall conclude, while the basic methodology of gathering the cost data is consistent, understanding additional factors is often subject to methodology.

\subsection{Customer Damage Function Estimation}

For the small-medium commercial and industrial database a similar set of procedures and analyses were conducted as those applied to the large commercial and industrial database. As with the large C\&I database, a Tobit regression model was used to estimate the relative effects of various independent variables rather than normal OLS regression. The same truncation of $0.05 \%$ of the highest values in the outage cost distribution and the transformation of the outage cost variable to a lognormal distribution were used. The distributions of the logged outage costs for the small-medium commercial and industrial customer database are shown in Figures 4-1 to 4-3. 


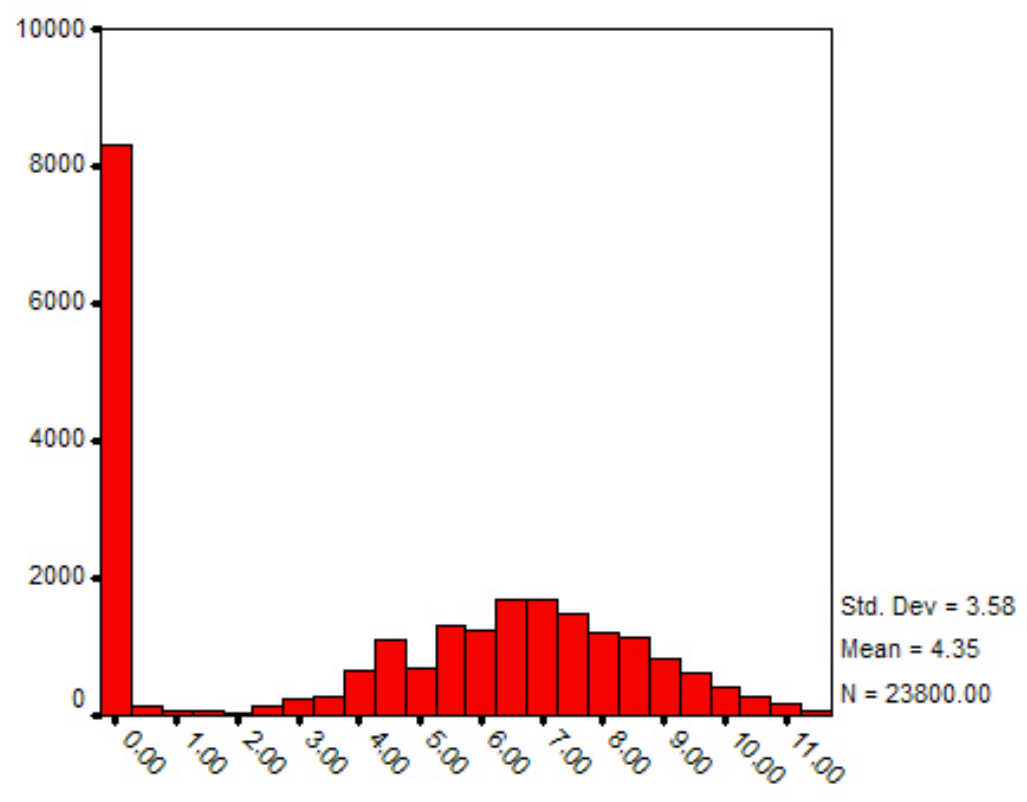

LNTCDEF

Figure 4-1. Logged Per Event Cost - Small-Medium C\&I

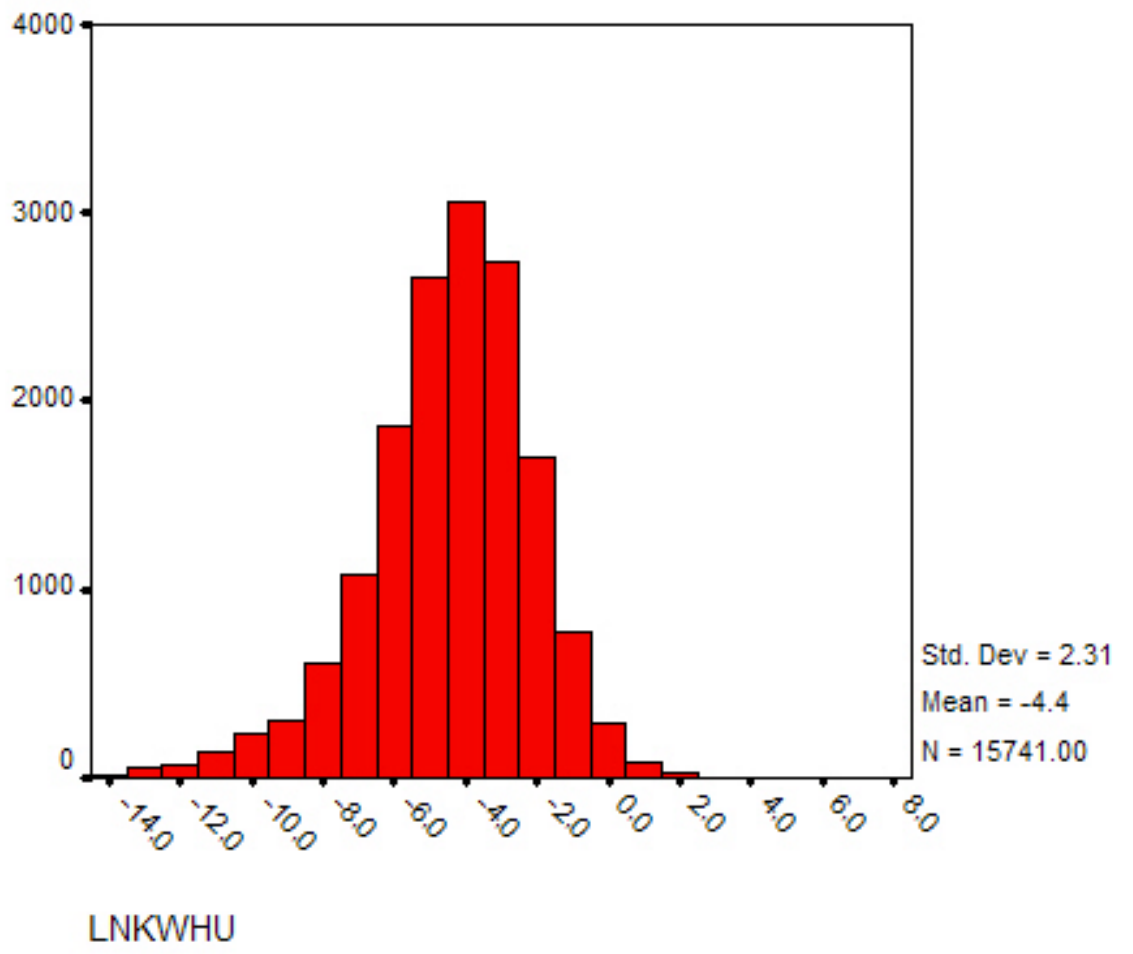

Figure 4-2. Logged Per Annual kWh Cost - Small-Medium C\&I 


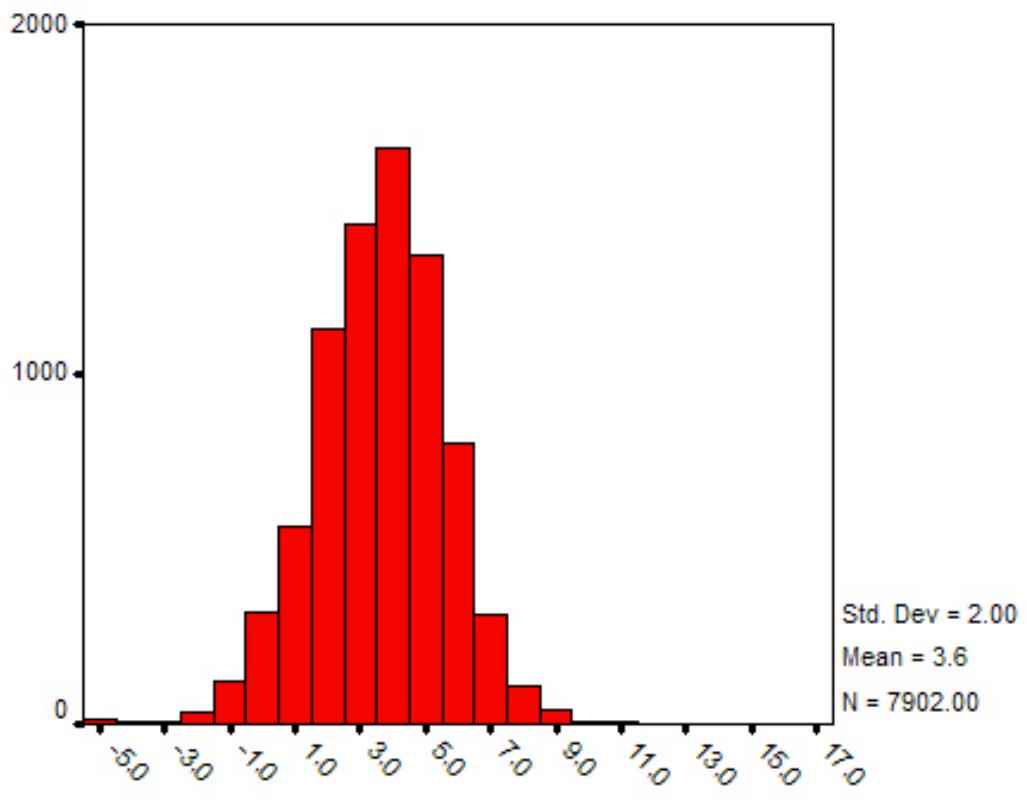

LNKWHD

\section{Figure 4-3. Logged Per Peak kW Cost - Small-Medium C\&I}

Table 4-4 below describes the two Tobit regression models that specify the relationship between various outage and customer characteristics and customer outage cost for this dataset. Model One contains only the parameters associated with outage characteristics and customer size as this has the largest number of cases available to estimate this model. Model Two includes parameters describing the business activities being interrupted. Information on the customers' SIC code was available for a smaller subset of the data. 
Table 4-4. Tobit Regression Models - Interruption Costs for Small-Medium C\&I

Tobit Regression Model Estimates

Small and Medium Commercial and Industrial Customer

Interruption Costs

\begin{tabular}{|c|c|c|c|c|c|c|}
\hline \multirow[b]{2}{*}{ Predictor } & \multicolumn{3}{|c|}{$\begin{array}{r}\text { Model One } \\
\text { Standard }\end{array}$} & \multicolumn{3}{|c|}{$\begin{array}{c}\text { Model Two } \\
\text { Standard }\end{array}$} \\
\hline & Parameter & Error & Probability & Parameter & Error & Probability \\
\hline Intercept & 6.48005 & 0.06525 & $<.0001$ & 5.92312 & 0.0851 & $<.0001$ \\
\hline Duration (hours) & 0.38489 & 0.01588 & $<.0001$ & 0.41996 & 0.02622 & $<.0001$ \\
\hline Duration Squared & -0.02248 & 0.0013408 & $<.0001$ & -0.02386 & 0.0019545 & $<.0001$ \\
\hline Number of Employees & 0.001882 & 0.0001749 & $<.0001$ & 0.0012817 & 0.0002144 & $<.0001$ \\
\hline Annual kWh & $1.703 \mathrm{E}-06$ & $1.2117 \mathrm{E}-07$ & $<.0001$ & $1.755 \mathrm{E}-06$ & $1.5918 \mathrm{E}-07$ & $<.0001$ \\
\hline Interaction Duration and $\mathrm{kWh}$ & 9.459E-08 & $2.547 \mathrm{E}-08$ & 0.0002 & 7.153E-08 & 2.7293E-08 & 0.0088 \\
\hline Morning & -0.6032 & 0.06151 & $<.0001$ & 0.22574 & 0.04755 & $<.0001$ \\
\hline Night & -0.91339 & 0.07035 & $<.0001$ & 0.95618 & 0.06147 & $<.0001$ \\
\hline Weekend & -0.52041 & 0.04657 & $<.0001$ & -0.26448 & 0.39049 & 0.4982 \\
\hline Winter & 0.37674 & 0.04154 & $<.0001$ & 0.60331 & 0.10596 & $<.0001$ \\
\hline Manufacturing & & & & -0.32852 & 0.10082 & 0.0011 \\
\hline Mining & & & & 0.11212 & 0.12045 & 0.3519 \\
\hline Construction & & & & -0.21343 & 0.05173 & $<.0001$ \\
\hline Transportation/Utilities & & & & -0.53278 & 0.1461 & 0.0003 \\
\hline Finance/Insurance Real Est. & & & & -0.32951 & 0.07094 & $<.0001$ \\
\hline Services & & & & -1.18103 & 0.09979 & $<.0001$ \\
\hline Public & & & & -0.39663 & 0.06814 & $<.0001$ \\
\hline Number of Observations & 12356 & & & 5555 & & \\
\hline Zero Response & 6637 & & & 2311 & & \\
\hline Log Likelihood & -23855 & & & -10336 & & \\
\hline
\end{tabular}

Figure 4-4, below, displays a comparison of the results of the customer damage function in Model One over the durations found in the sample dataset under varying times of day and seasons. The results show that the relationship between damage and duration is non-linear for small and medium customers as it was for large customers, albeit at much lower average values. Costs increase slowly within the first hour; accelerate through the second through the eighth hours; and, again, decline thereafter. All of the predictions are positive at the intercept representing the cost of momentary interruptions. Outage costs for winter outages are significantly higher than those experienced in summer; and outage costs during the night and on weekends are significantly lower as expected. The results show that an average small-medium customer in terms of its number of employees and consumption will have approximately $\$ 1,200$ in costs for a 1-hour summer afternoon outage and $\$ 1,800$ for a 1-hour winter afternoon outage. 


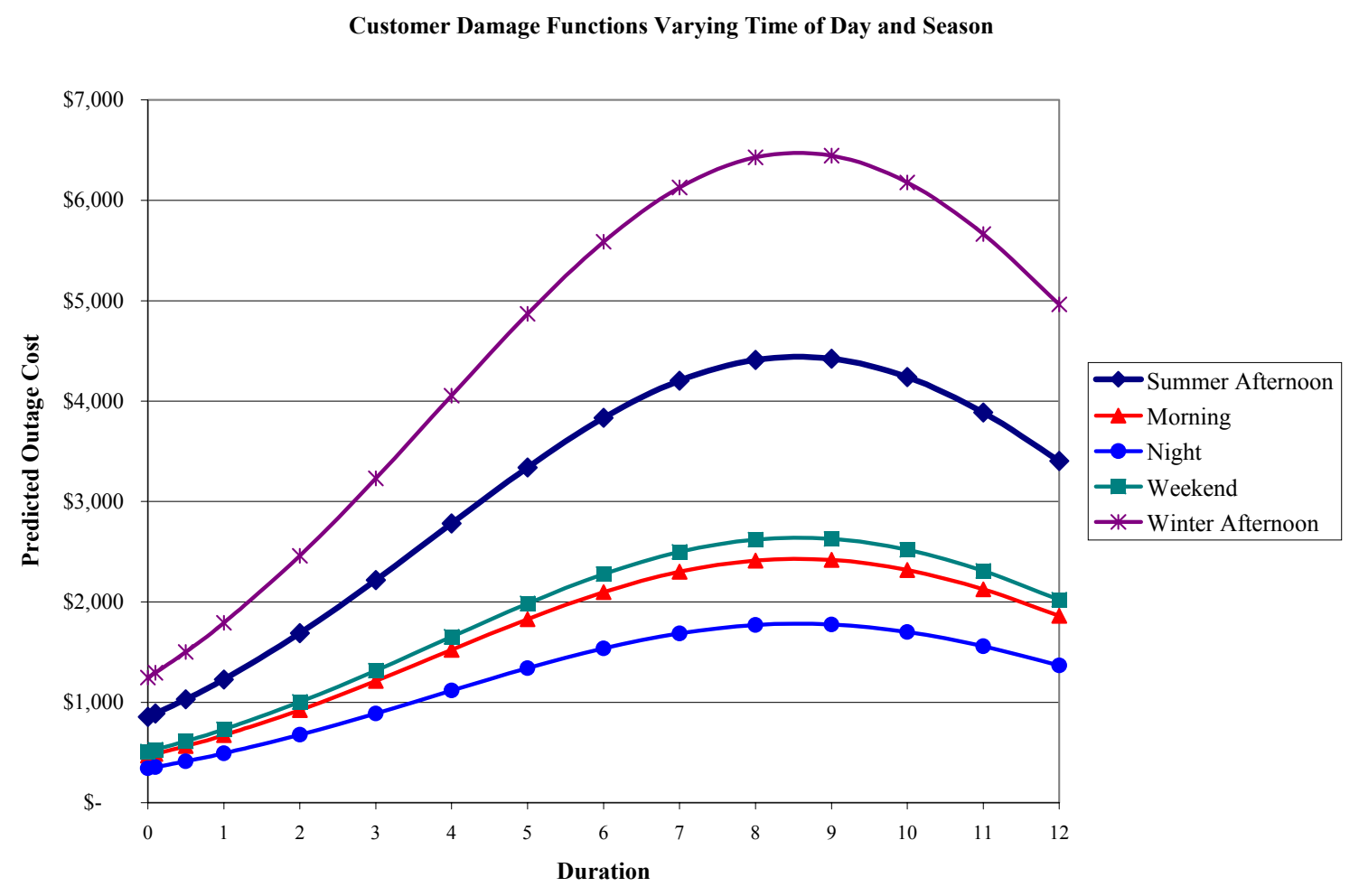

Figure 4-4. Customer Damage Functions - Model One Varying Season and Time of Day

Figure 4-5, below, displays the results of estimating customer outage costs for summer afternoons varying annual $\mathrm{kWh}$ consumption away from the mean by a factor of 10 (i.e. from $11,293 \mathrm{kWh}$ (small) to 1,182,930 (relatively large)). The size of customer's load has a substantial impact on outage costs and the relationship again is not linear. Decreasing $\mathrm{kWh}$ consumption by $90 \%$ from the average consumption in the sample results in only a small reduction in customer interruption cost, while increasing it by a factor of 10 dramatically increases customer interruption cost. 
Customer Damage Functions Varying Customer Size

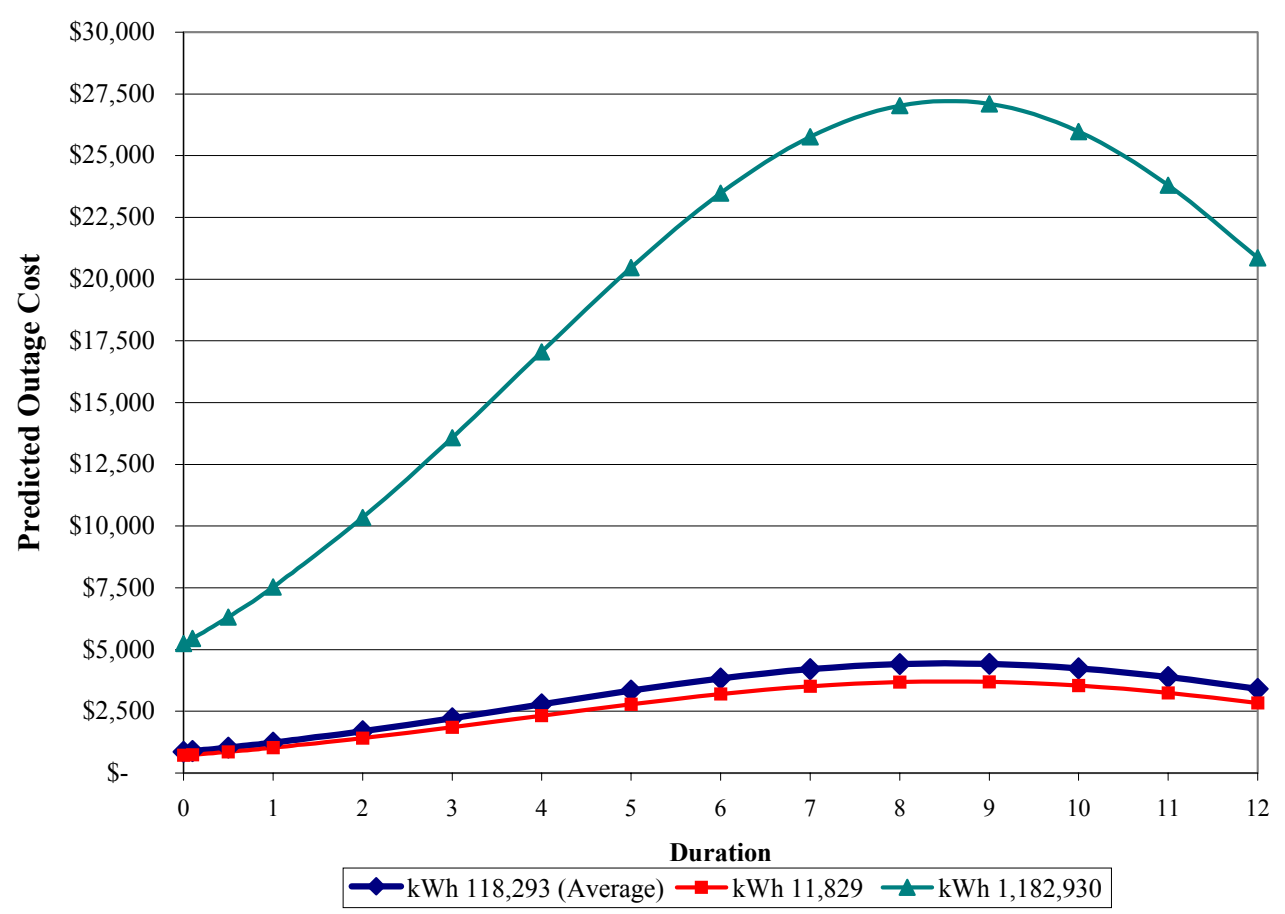

Figure 4-5. Customer Damage Functions - Model One Varying Customer Size

In addition to outage attributes and customer size, Model Two contains parameters describing the effects of business type on interruption cost. A separate model was estimated for this analysis because several of the surveys included in the study did not report business type. Figure 4-5, below, displays the customer damage functions from Model Two estimated for different types of businesses. All of the other parameters in the model are held at their means thus allowing an assessment of the independent effect of business type. That is, the dark blue line (with the diamond marks) indicates the customer damage function for outages on a summer afternoon, experienced by establishments involved in retail trade using an average of $118,000 \mathrm{kWh}$ annually and with about 22 employees.

The results indicate that outage costs for manufacturing far outstrip those of any other business activity in the small and medium customer class. They are more than double the costs experienced by businesses involved in retail trade and more than four times the cost experienced by customers involved in public sector activities (e.g., schools and municipal buildings). The next highest outage costs are for customers involved in construction. Costs for manufacturing and construction are significantly higher than those of other businesses because they depend heavily on electricity to directly support production. Costs for other business types are relatively close to those of retail trade - though the differences among them are statistically significant. 
Customer Damage Functions Varying Business Type

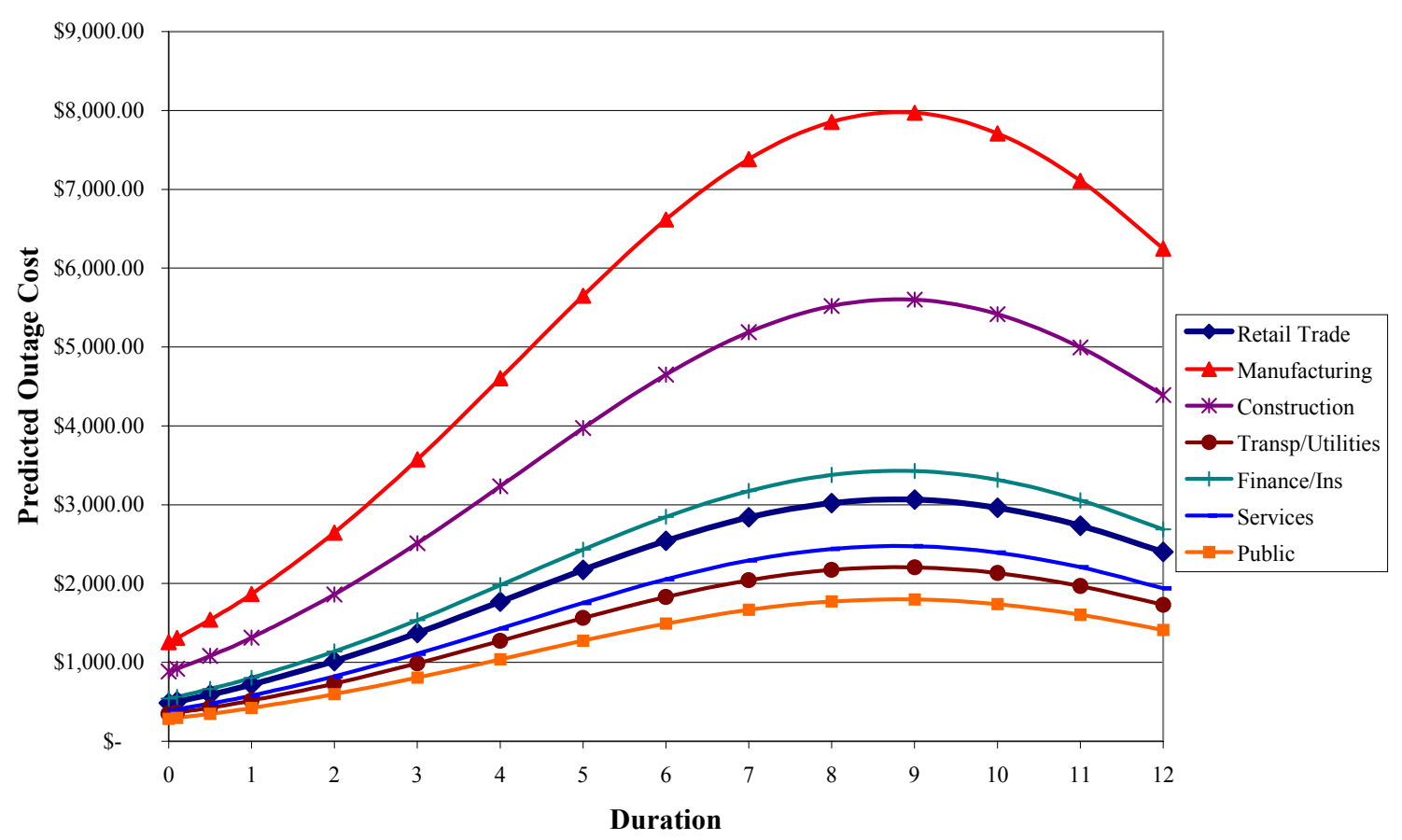

Figure 4-6. Customer Damage Functions For Various Business Types

As with the large commercial and industrial model, the results for the small-medium model appear reasonable. As with the large C\&I model, one can use the customer damage functions from the above models to estimate customer interruption costs under a wide variety of conditions. Again, these models cannot be used to estimate damages for individual companies. The regression functions used are predicting the mean of the customer outage cost for populations of customers with different characteristics under different conditions. There is substantial unexplained variation among customers in the outage costs they experience resulting from factors that are not accounted for in the above equations (e.g., process design differences, resistance of equipment to electric disturbances, etc.) and will not generally be known without an in-depth interview. The existence of these unknowns virtually guarantees that the prediction for any individual customer from the above functions will be significantly in error.

Again, this caution does not apply to the application of the above equations for reasonably large populations of customers where the law of large numbers and central limit theorem ensure that random but significant differences among customers do not produce estimates that deviate dramatically from the predictions made by the above equations. 


\section{Residential Results}

The residential database is somewhat different from the two commercial and industrial databases. The most important difference is that most residential studies of outage costs or value of service do not focus on "direct worth or cost" estimates; rather they use a willingness to pay or willingness to accept measures. Developing these measures generally involves describing a scenario to a residential customer and then ask them what they would be willing to pay to avoid this specific outage or what they would be willing to accept as compensation (usually described as a credit on their bill) in order to put up the outage. The primary reason for using these alternatives to direct cost is the assumption that much of the "cost" of an outage for residential customers is associated with the hassle, inconvenience, and personal disruption of the outage, rather than direct out-of-pocket expenses, like buying candles. In this situation, customers may be able to more accurately represent the value of reliability by expressing their willingness to pay to avoid an outage (or their willingness to accept some type of credit to accept an outage) rather than calculate an out of pocket cost or savings.

In theory from an economic perspective, willingness to pay (WTP) and willingness to accept (WTA or Credit) measures should produce the same value for a given outage. In practice, it is difficult to construct questions that produce identical results. Customers tend to place paying the utility in a different frame of reference than accepting a credit from the utility. Typically, willingness to accept measures produce a higher estimated value than willingness to pay measures. There are various practical and theoretical reasons offered for this finding. As a practical matter for this meta-analysis, all of the studies used a WTP framework and only a few also tested a WTA framework. Consequently the analysis focused on the WTP results (although the WTA or Credit results are shown in some tables if they were available).

In addition to the differences in measuring outage costs, the residential sector is also a much more homogenous population with respect to outage costs. Where commercial and industrial customer studies find outages costs from 0 to millions of dollars, the typical residential study shows that outage costs vary over a much smaller range depending on the scenario. This effectively reduces the variation in the outage cost variable making it somewhat more difficult to find powerful explanatory variables. Households themselves are also more homogenous than business customers in terms of the end uses, dependence on electricity for critical operations, and consumption. This is not to say that reliability is not critically important to residential customers, rather to note that the range of variation in outage costs and in customer characteristics is much narrower in the residential sector.

The residential database was built from 6 studies conducted by 5 companies, with a total of 11,368 respondents. There were approximately 28,800 individual responses to scenarios that form the basis of the merged dataset, subject to availability as a result of missing data. Table 51 , below, shows the distribution of responses available for analysis by region, season, day of the week, and year. 
Table 5-1. Number of Responses by Region, Season, Day of Week, and Year

\begin{tabular}{|c|c|c|c|c|c|c|c|c|}
\hline \multirow[b]{2}{*}{ Region - Company } & \multirow[b]{2}{*}{ Season } & \multirow[b]{2}{*}{ Day of Week } & \multicolumn{6}{|c|}{ Year of Survey } \\
\hline & & & 1989 & 1993 & 1997 & 1999 & 2000 & Total \\
\hline \multirow[t]{3}{*}{ Northwest-1 } & Winter & Weekday & 1033 & & & & & 1033 \\
\hline & Summer & Weekday & 519 & & & & & 519 \\
\hline & Winter & Weekend & 514 & & & & & 527 \\
\hline \multirow[t]{3}{*}{ Southeast-2 } & Winter & Weekday & & 527 & & & & 9257 \\
\hline & Summer & Weekday & & 4213 & 5044 & & & 860 \\
\hline & Summer & Weekend & & & 748 & & & 748 \\
\hline Northwest-2 & Winter & Weekday & & & & & & 12431 \\
\hline \multirow[t]{4}{*}{ Southwest } & Summer & Weekday & & & & & & 1781 \\
\hline & Winter & Weekday & & & & & 1808 & 1808 \\
\hline & Summer & Weekday & & & & & 7856 & 7856 \\
\hline & Summer & Weekend & & & & & 904 & 904 \\
\hline \multirow[t]{3}{*}{ West } & Winter & Weekday & & & & & 1362 & 1362 \\
\hline & Summer & Weekday & & & & & 3398 & 3398 \\
\hline & Winter & Weekend & & & & & 688 & 688 \\
\hline \multicolumn{3}{|l|}{ TOTAL } & 2066 & 4740 & 5742 & 14212 & 16016 & 42826 \\
\hline
\end{tabular}

Table 5-2. Average Outage Costs by Duration BY DURATION

\begin{tabular}{|c|c|c|c|c|c|c|c|}
\hline \multicolumn{8}{|c|}{ Willingness to Pay } \\
\hline Duration & $\overline{\mathbf{N}}$ & \# Firms & Median & Average & Std Dev & $25 \%$ & $75 \%$ \\
\hline All & 28042 & 5 & 1.40 & 6.49 & 10.69 & - & 7.31 \\
\hline $1-2 \mathrm{sec}$ & 2472 & 2 & 2.70 & 5.84 & 9.07 & 0.62 & 6.23 \\
\hline $10 \mathrm{sec}$ & 1738 & 1 & - & 2.32 & 6.58 & - & 1.04 \\
\hline $30 \mathrm{~min}$ & 3546 & 1 & - & 5.81 & 10.97 & - & 5.22 \\
\hline $1 \mathrm{hr}$ & 11200 & 5 & 2.24 & 6.90 & 10.85 & - & 10.45 \\
\hline $2 \mathrm{hr}$ & 370 & 1 & 6.23 & 7.39 & 8.53 & 2.49 & 9.34 \\
\hline $4 \mathrm{hr}$ & 6872 & 4 & 2.61 & 7.14 & 11.39 & - & 10.45 \\
\hline $8 \mathrm{hr}$ & 1637 & 2 & 1.45 & 5.15 & 8.41 & - & 7.26 \\
\hline $12 \mathrm{hr}$ & 207 & 1 & 21.60 & 26.27 & 11.55 & 16.20 & 32.40 \\
\hline \multicolumn{8}{|c|}{ Credit } \\
\hline & $\mathbf{N}$ & \# Firms & Median & Average & Std Dev & $25 \%$ & $75 \%$ \\
\hline$\overline{A \text { All }}$ & 12,615 & 5 & 5.61 & 10.24 & 14.54 & 0.56 & 12.45 \\
\hline $1-2 \mathrm{sec}$ & 2,849 & 2 & 1.12 & 5.07 & 9.49 & - & 5.61 \\
\hline $10 \mathrm{sec}$ & & 1 & & & & & \\
\hline $30 \mathrm{~min}$ & & 1 & & & & & \\
\hline $1 \mathrm{hr}$ & 6,308 & 5 & 5.61 & 10.52 & 13.52 & 1.12 & 12.45 \\
\hline $2 \mathrm{hr}$ & 475 & 1 & 6.23 & 8.99 & 11.65 & 1.25 & 12.45 \\
\hline $4 \mathrm{hr}$ & 1,880 & 4 & 6.23 & 12.49 & 15.54 & 2.24 & 16.82 \\
\hline $8 \mathrm{hr}$ & 972 & 2 & 7.26 & 14.77 & 18.77 & 0.73 & 17.78 \\
\hline $12 \mathrm{hr}$ & 131 & 1 & 43.20 & 47.81 & 28.44 & 27.00 & 80.99 \\
\hline
\end{tabular}

Table 5-2 shows that residential consumers generally report increasing WTP and WTA as the length of the outage increases. As with the commercial and industrial dataset, it is useful to see the underlying average costs embedded in the data for customers who responded to the various scenarios. Results for both WTP and WTA questions are shown. In terms of duration, Table 5-2 
shows that residential consumers generally report increasing WTP and WTA as the length of the outage increases.

However, the data are inconsistent and the standard deviations are generally larger than the average. The inconsistency suggests that the outage costs reported by customers tend to vary widely across the studies and the average outage costs for any given duration are subject to a great deal of influence from the studies used for that scenario.

The two most robust estimates for duration are the 1-hour and 4-hour as these two scenario durations were used in multiple studies across multiple regions. The average cost per event for the 1-hour using a WTP methodology is $\$ 6.90$ and the average for a 4-hour is $\$ 7.14$, suggesting only a modest impact of duration on residential customer's willingness to pay to avoid an outage. Using a WTA methodology, the average cost for the 1-hour is $\$ 10.52$ and the average cost for the 4-hour is $\$ 12.49$. In both cases, however, the standard deviations are fairly large compared to the mean and the median values tend to be lower than the average.

The outage costs measured as both WTP and WTA for several other key variables are shown in Table 5-3. All costs are for scenarios with 1-hour duration, but they include a range of other attributes like winter versus summer and time of day. Overall, the results suggest that outage costs per event for residential customers are:

- Higher in the winter than in the summer;

- Higher on weekends than on weekdays (reversing the trend for commercial and industrial customers);

- Fairly constant across the regions with the exception of the West region;

- Fairly constant across customers living in different dwelling types; and

- Fairly constant across homeowners and renters.

While these patterns are generally consistent with results from individual studies of outage costs, caution must be used in interpreting the point estimates as different groups of customers responded to different combinations of scenario attributes. 
Table 5-3. Average Outage Costs for Key Variables (WTP and WTA)

1 HOUR DURATION

\begin{tabular}{|c|c|c|c|c|c|c|}
\hline \multicolumn{7}{|c|}{ Willingness to Pay } \\
\hline SEASON & $\mathbf{N}$ & Median & Average & Std Dev & $25 \%$ & $75 \%$ \\
\hline$\overline{\text { All }}$ & 11200 & 2.24 & 6.90 & 10.85 & - & 10.45 \\
\hline Winter & 2408 & 1.45 & 8.24 & 12.26 & - & 10.80 \\
\hline Summer & 8792 & 2.24 & 6.54 & 10.41 & - & 7.47 \\
\hline \multicolumn{7}{|c|}{ Willing to Accept } \\
\hline All & 6,308 & 5.61 & 10.52 & 13.52 & 1.12 & 12.45 \\
\hline Winter & 1,141 & 10.80 & 15.38 & 15.83 & 2.70 & 27.00 \\
\hline Summer & 5,167 & 5.61 & 9.45 & 12.71 & 1.12 & 11.21 \\
\hline \multicolumn{7}{|c|}{ Willingness to Pay } \\
\hline DAY & $\mathbf{N}$ & Median & Average & Std Dev & $25 \%$ & $75 \%$ \\
\hline All & 11200 & 2.24 & 6.90 & 10.85 & - & 10.45 \\
\hline Weekday & 10557 & 1.57 & 6.86 & 10.91 & - & 9.96 \\
\hline Weekend & 643 & 5.61 & 7.71 & 9.79 & 1.12 & 11.21 \\
\hline \multicolumn{7}{|c|}{ Willing to Accept } \\
\hline All & 6,308 & 5.61 & 10.52 & 13.52 & 1.12 & $\overline{12.45}$ \\
\hline Weekday & 5,632 & 5.61 & 10.48 & 13.52 & 1.12 & 12.45 \\
\hline Weekend & 676 & 5.61 & 10.83 & 13.53 & 1.12 & 11.21 \\
\hline \multicolumn{7}{|c|}{ Willingness to Pay } \\
\hline Region & $\mathbf{N}$ & Median & Average & Std Dev & $25 \%$ & $75 \%$ \\
\hline All regions & 11200 & 2.24 & 6.90 & 10.85 & - & 10.45 \\
\hline Northwest & 2243 & 2.70 & 7.60 & 10.70 & 0.54 & 10.80 \\
\hline Southwest & 4380 & - & 7.02 & 12.78 & - & 10.45 \\
\hline Southeast & 3903 & 5.60 & 7.17 & 8.97 & 1.12 & 11.21 \\
\hline West & 674 & - & 2.26 & 5.13 & - & 3.13 \\
\hline \multicolumn{7}{|c|}{ Willing to Accept } \\
\hline All regions & 6,308 & 5.61 & 10.52 & 13.52 & 1.12 & 12.45 \\
\hline Northwest & 1,782 & 7.26 & 13.46 & 15.38 & 1.45 & 21.60 \\
\hline Southwest & & & & & & \\
\hline $\begin{array}{l}\text { Southeast } \\
\text { West }\end{array}$ & 4,526 & 5.61 & 9.36 & 12.53 & 1.12 & 11.21 \\
\hline \multicolumn{7}{|c|}{ Willingness to Pay } \\
\hline $\begin{array}{l}\text { Home } \\
\text { Ownership }\end{array}$ & $\mathbf{N}$ & Median & Average & Std Dev & $25 \%$ & $75 \%$ \\
\hline All & 11071 & 2.24 & 6.90 & 10.86 & - & 10.45 \\
\hline Own & 8874 & 2.24 & 6.93 & 10.81 & - & 10.45 \\
\hline Rent & 2028 & 1.45 & 6.55 & 10.65 & - & 7.26 \\
\hline Other & 169 & 2.61 & 9.49 & 14.79 & - & 15.67 \\
\hline \multicolumn{7}{|c|}{ Credit } \\
\hline All & 6,158 & 5.61 & 10.44 & 13.48 & 1.12 & 12.45 \\
\hline Own & 4,934 & 5.61 & 10.36 & 13.47 & 1.12 & 12.45 \\
\hline Rent & 1,191 & 5.61 & 10.72 & 13.37 & 1.25 & 14.51 \\
\hline Other & 33 & 3.36 & 12.25 & 18.00 & 0.73 & 12.45 \\
\hline
\end{tabular}




\subsection{Customer Damage Function Estimation}

To account for the influences of different outage and customer characteristics, a multivariate analysis of the residential data was conducted. Similar to the commercial and industrial data, a Tobit framework was used to deal with the truncated nature of the dependent variable. To some extent this issue is even more problematic with residential customers because the percent of customers giving a " 0 " response can be as high as 60 to 80 percent for short duration outages. Use of the Tobit model allows for the estimation of unbiased parameters to measure the relative effects of the outage attributes and customer characteristics given the high number of 0 responses.

For the modeling, the analysis also focused on the WTP estimates of outage costs instead of the WTA. There are two reasons. The most important is that there is more data across the studies in which a WTP framework was used. Second, the WTP estimates have been more generally accepted as providing a more accurate assessment of the value of service reliability.

The same basic treatment of the dependent variable used in the commercial and industrial datasets is also used for the residential data. The dependent variable - the willingness to pay measure - was transformed using a log transformation prior to the Tobit regression. The distribution of willingness to pay values in the residential sample is skewed toward zero, but includes a number of high values. The log transformation helps assure that the distribution of the error term is normal (an important assumption in the use of the Tobit model). Again, the top $.05 \%$ of the WTP and WTA distributions were truncated.

Table 5-4 shows the basic model developed from the residential data. This model used the maximum available data across the studies since most of the studies included household income, $\mathrm{kWh}$ annual usage, and region along with the outage attribute variables. In constructing the model, it was determined that a $\log$ transformation of the household income variable was also required to account for the skewed nature of the income distribution and the nonlinear nature of the relationship between income and outage costs.

The model uses several dummy variables (variables coded as 0 or 1 meaning the presence or absence of that characteristic) to assess the effects of key variables. The suppressed categories for the model are the Northwest for the region variable and summer afternoon for the outage attributes (e.g., if no value is specified for morning, night, weekend, or winter, then the model is simulating a summer afternoon). 


\section{Table 5-4. Tobit Regression for the Residential Data, Willingness-To-Pay}

\begin{tabular}{|l|rr|}
\hline Predictor & Parameter & Probability \\
\hline Intercept & 0.2503 & 0.1468 \\
Duration & 0.2211 & $<.0001$ \\
Duration Squared & -0.0098 & $<.0001$ \\
Morning & -0.0928 & 0.0061 \\
Night & -0.1943 & $<.0001$ \\
Weekend & -0.0134 & 0.7454 \\
Winter & 0.1275 & 0.0006 \\
Annual MWh (kWh/1000) & 0.0065 & $<.0001$ \\
Log of Household Income & 0.0681 & $<.0001$ \\
Southeast & 0.2015 & $<.0001$ \\
West & -0.115 & 0.0228 \\
Southwest & 0.5256 & $<.0001$ \\
& \multicolumn{3}{|c}{} \\
N & \multicolumn{3}{|c}{12057} \\
Zero Responses & 7319 \\
Log-Likelihood & -20868 \\
\hline
\end{tabular}

This model can be used to estimate outage cost for various combinations of the independent variables. As with the commercial and industrial models, this model predicts the log of the outage cost value and hence the antilog must be taken of the predicted value to convert it to a predicted outage cost. Figure 5-1, below, shows the predicted outage costs across various durations for the four regions for a summer afternoon outage. The results show that outage costs are lowest in the West and highest in the Southwest with the Northwest and Southeast having intermediate values. The model predicts that the values for a momentary outage (duration set to 0 ) range from $\$ 1.91$ per event in the West to $\$ 3.23$ in the Southwest. At a 1-hour duration, the values range from $\$ 2.10$ to $\$ 4.00$ per event. For the 8-hour duration, the values have slightly more than doubled to a range of $\$ 5.34$ to $\$ 10.13$ per event.

On the face of it, the model results fit with the range of values expected from other outage cost studies. Estimated willingness to pay is approximately $\$ 2.00$ to $\$ 4.00$ per event for a 1 -hour outage. The fact that the Southwest has the highest costs on a summer afternoon and that these values increase more steeply with duration is consistent with what would be expected in a desert climate. Similarly, the West or Northwest, with more of their area in the moderate coastal climates, could reasonably be expected to have lower values. 


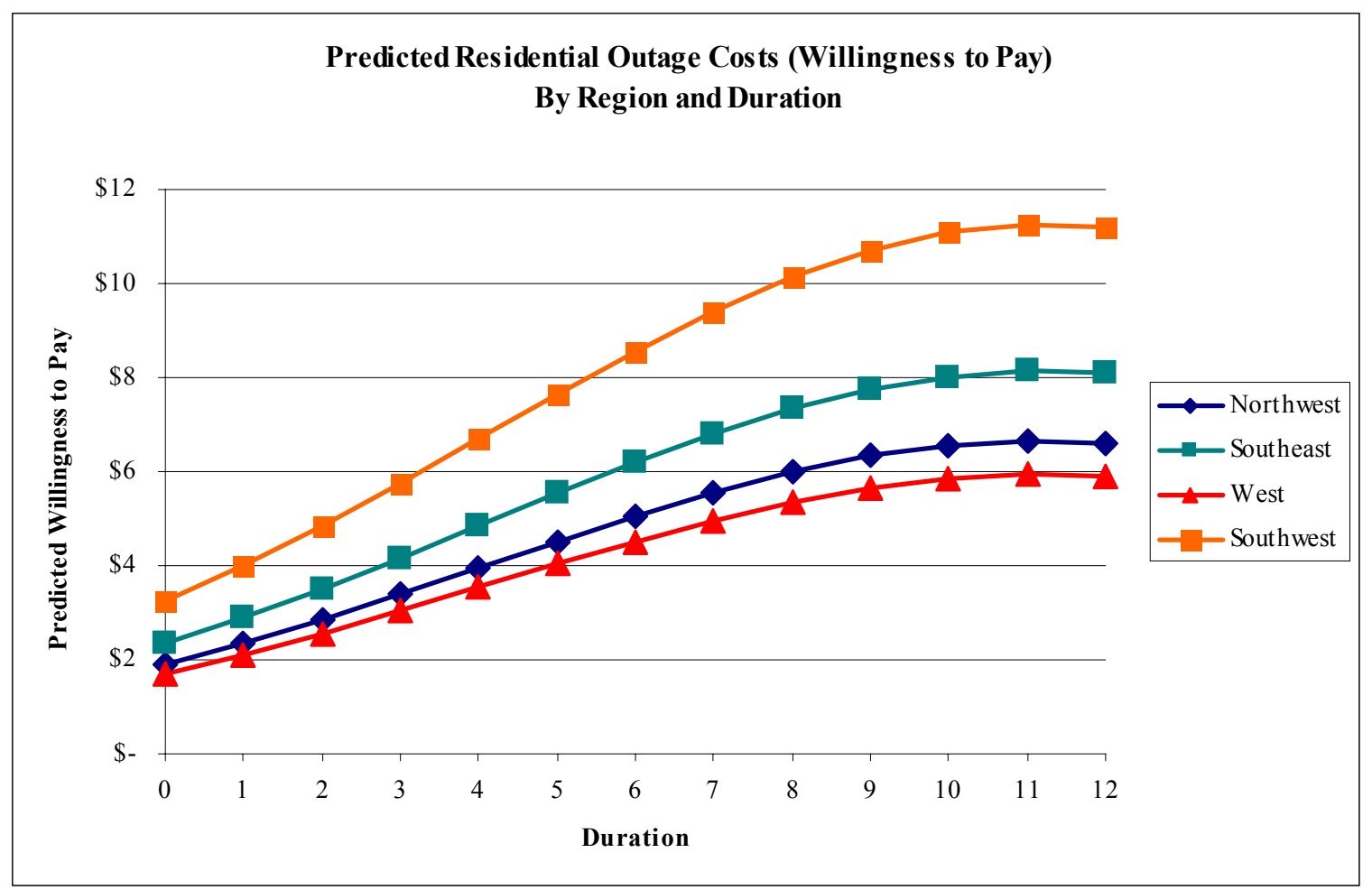

Figure 5-1. Predicted Outage Cost By Region and Duration

While the predicted outage costs make sense and have a plausible interpretation, it is also important to point out that in this analysis, the region is closely associated with the individual study. Since most regions are based on a single utility study, the results may be confounding the effects of methodological differences, climate differences, and unique market conditions. Also, as noted previously, the results do not include any data from the Midwest or Northeast.

The model can be used to estimate the impact of other combinations of scenario and customer characteristics as well. Figure 5-2 reports estimates of the effects of winter versus summer outage costs for three regions by starting with a morning outage and varying duration. Overall, winter outages tend to have higher outage costs than summer outages and the difference varies from an increase of $\$ 0.25$ to $\$ 0.50$ for a 1 -hour outage to about $\$ 0.75$ to $\$ 1.50$ for an 8 -hour outage, depending on the region. This simulation, however, also points to difficulty in using the combined studies to pick up subtle effects. In particular, the model is forecasting a higher outage cost value for a winter scenario in the Southwest than a summer scenario, but original data from the study done in the Southwest suggests that summer outages have higher values than winter (although not significantly higher). The effect of aggregating the data across studies blurs some of these differences. 


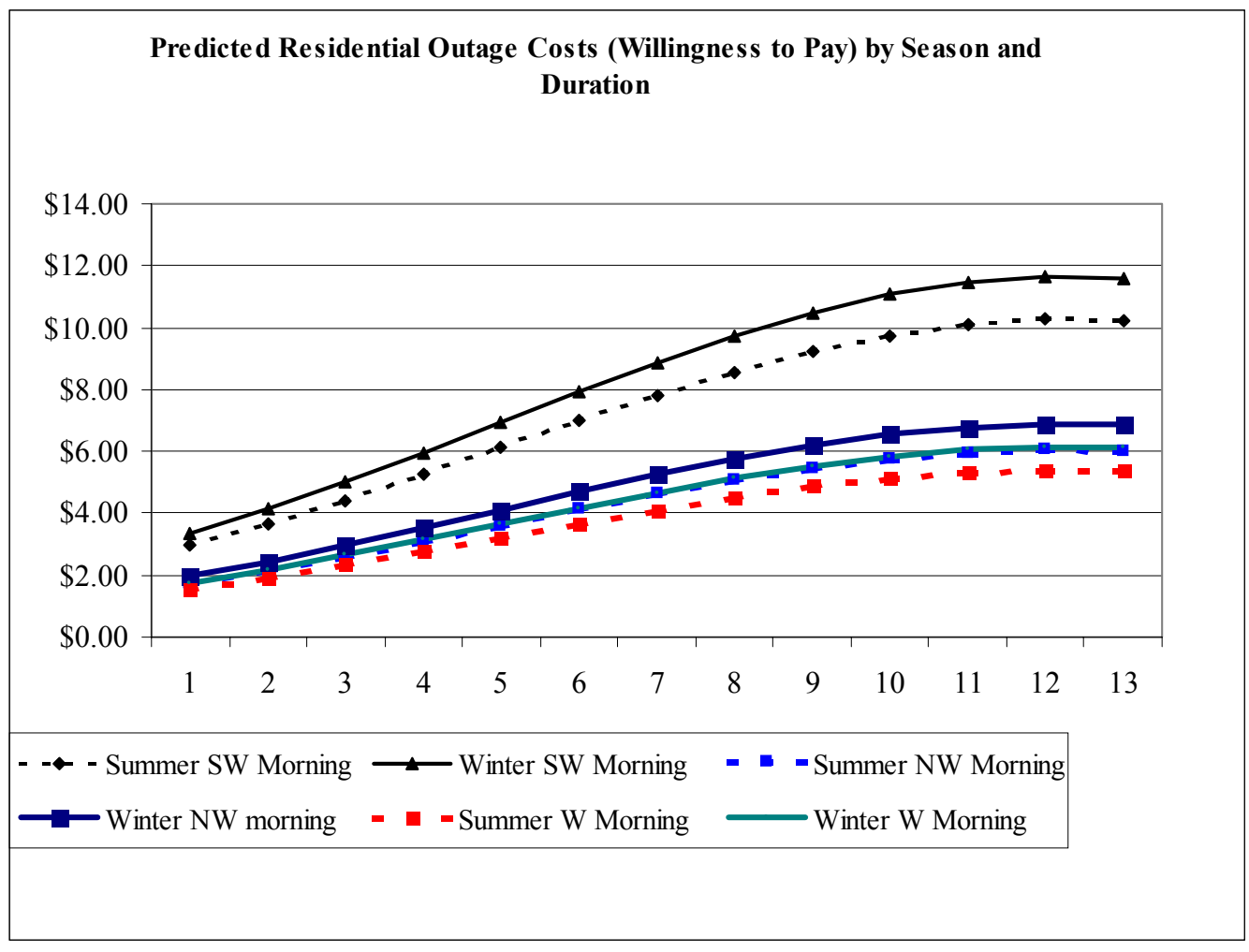

Figure 5-2. Predicted Outage Cost By Region and Duration

Figure 5-3 shows a simulation of outage costs for households with low versus high annual consumption, where low consumption was defined as less than 4,000 kWh per year and high was defined as greater than $15,000 \mathrm{kWh}$ per year. The simulation shows a small effect of household energy consumption on predicted outage costs. The difference between a low consumption household and a high consumption household ranges from $\$ 0.2$ to $\$ 0.4$ for a 1-hour outage to $\$ 0.6$ to $\$ 1.0$ for an 8 -hour outage. The effect is significant, but it is not a large effect compared to the variation across regions.

The results from combining the data across the residential studies for this meta-analysis are encouraging but require further work to clarify the value of service reliability in this sector. The most encouraging aspect is that it appears that data from several studies can be reasonably combined to test the effects of various outage attributes and customer characteristics across a broader geography and range of outage scenarios than is possible in individual studies. The combined results, particularly when controlled in a multivariate analysis, are fairly consistent in the prediction of outage cost values across various durations, and the results are plausible. Overall, the models show average 1-hour summer afternoon outage costs for residential customers in the $\$ 2$ to $\$ 4$ range, an estimate that is not substantially different than other efforts to estimate this cost, yet it is based on combining data across several studies with slightly different methodologies and from different parts of the country. Further, the estimates along the duration curve and the variation across types of characteristics are generally sensible given what is known about outage costs. 


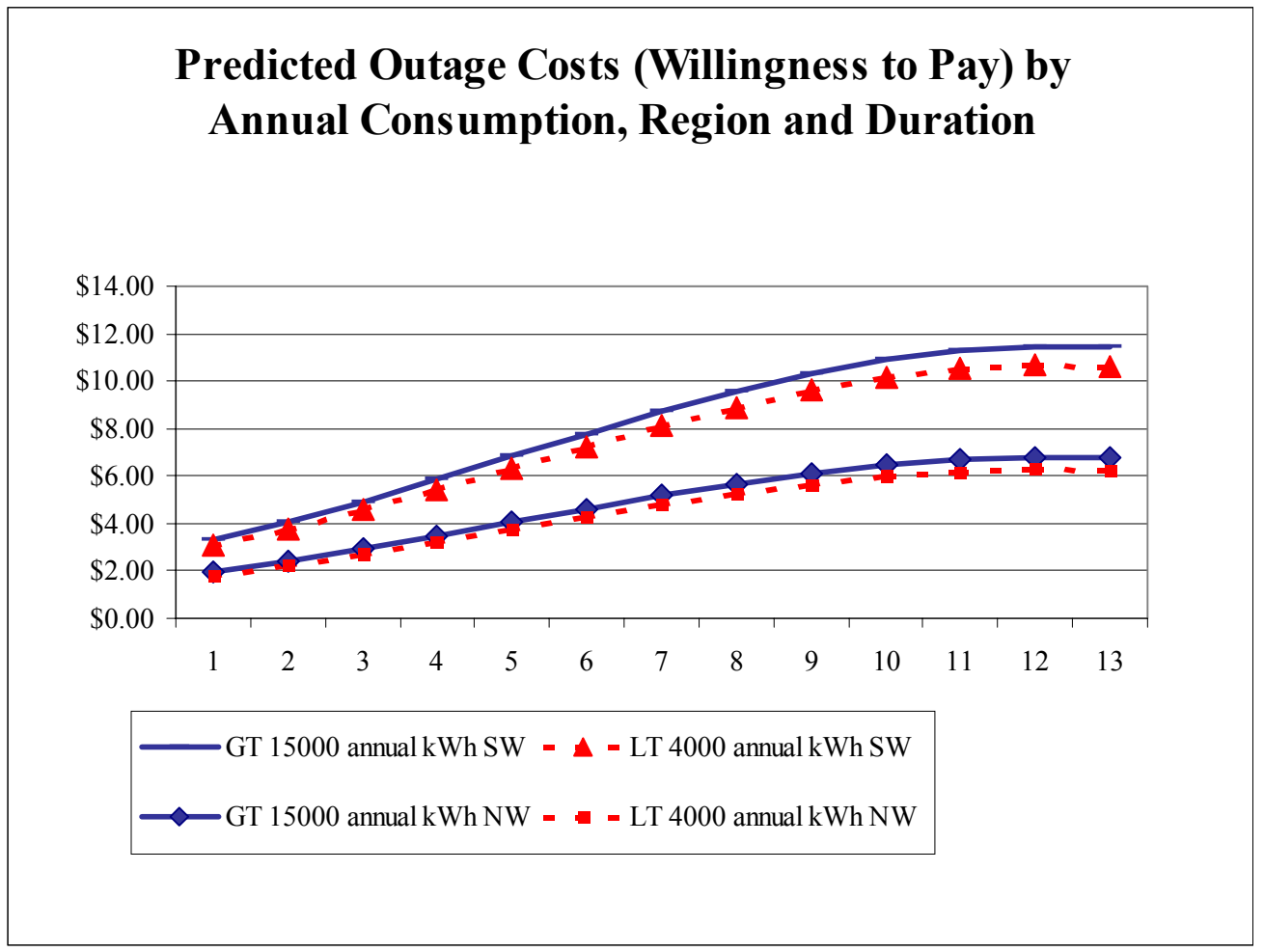

Figure 5-3. Predicted Outage Cost By Region and Duration

The conclusion from this effort is that the model is producing reasonable estimates of outage costs from residential customers, which can be meaningfully used in the analysis of costs and benefits of various policy or investment decisions as they related to increasing or decreasing customer's experiences with reliability (as manifest in outages with various characteristics). However, the model and the results of the meta-analysis are not without important caveats. Collinearity is the primary caveat. The combined dataset lacks sufficient data such that region, methods of outage cost measurement, outage attributes, weather, and local market conditions would vary independently, thereby allowing for a complete disaggregation of the effects of these variables. In addition to problems with the collinear nature of the data in the meta-analysis, the results also show that there is insufficient data for some key variables, which precludes the effects of those variables from being tested. For example, data on household composition and the presence or absence of backup devices could not be included in the prediction model because these variables were not available for the majority of the cases. Addressing these two issues will require future studies that collect data more comprehensively across the U.S. rather than what exists today as a series of independent studies by utilities. 


\section{Summary and Conclusions}

Reliable electric power is a salient underpinning of the U.S. economy and a central focus of stakeholders in the electric power industry. While no one disagrees that customers seek reliable power, the strategies available to meet that goal are numerous and the price tags associated with various ultimate strategies can be quite high. Underlying any strategy are critical assumptions about the value ultimate customers place on reliability. During times of crisis, the assumption is that consumers will pay almost any price for reliable power. During periods of reliable power delivery there are frequent charges that the system is being overbuilt and designed to a higher standard of reliability than customers are willing to pay.

Over the past 20 years, there have been numerous studies representing millions of dollars of research designed to measure the value of service reliability to customers. Among these efforts is a group of about 30 studies conducted by 12 utilities that used a customer survey based approach to measuring outage costs. These studies represent the largest body of research in this area that has similarities in the approach and measurement protocols. However, in terms of developing a generalized model of outage costs, comparisons among these studies have been frustrating. Though most studies report certain essential information such as the cost of a one hour outage or the costs to core customers segments, the information is difficult to compare because of underlying differences in the customer base, market conditions, and nature of the outage scenarios examined.

This project is an attempt to address this limitation by acquiring and merging the various datasets into a larger integrated set of data for analysis. Of the 30 or so identifiable studies using one of these survey-based approaches, 25 of the datasets were acquired by the project team. After reviewing the variables and developing common metrics, a total of 24 studies were used to develop three outage costs datasets - a large commercial and industrial customer dataset (customer greater than $1 \mathrm{MW}$ of demand); a small and medium commercial and industrial dataset; and a residential dataset (Table 6-1). (In cases where the cells are merged, there was one study but the respondents were separated by usage into either small-medium or large C\&I.)

\section{Table 6-1. Summary of Studies Used in the Meta-analysis}

\begin{tabular}{|c|c|c|c|c|}
\hline Company & $\begin{array}{c}\text { Survey } \\
\text { Year }\end{array}$ & $\begin{array}{c}\text { Large } \\
\text { C\&I }\end{array}$ & $\begin{array}{c}\text { Small / } \\
\text { Medium } \\
\text { C\&I }\end{array}$ & Residential \\
\hline Southeast-1 & 1997 & \multicolumn{2}{|c|}{ X } & \\
\hline \multirow[t]{2}{*}{ Southeast-2 } & 1993 & $X$ & $X$ & $\mathrm{X}$ \\
\hline & 1997 & $X$ & $X$ & $X$ \\
\hline \multirow[t]{2}{*}{ Southeast-3 } & 1990 & $\mathrm{X}$ & $\mathrm{X}$ & \\
\hline & 1991 & $X$ & & \\
\hline Midwest & 2002 & $X$ & $X$ & \\
\hline West & 2000 & $X$ & $\mathrm{X}$ & $\mathrm{X}$ \\
\hline Southwest & 2000 & $\mathrm{X}$ & $\mathrm{X}$ & $\mathrm{X}$ \\
\hline Northwest-1 & 1989 & \multicolumn{2}{|r|}{$X$} & $X$ \\
\hline Northwest-2 & 1999 & $X$ & $X$ & $X$ \\
\hline
\end{tabular}


The resulting data provide a resource for developing more generalized estimates of outage costs but each dataset also has limitations. It was possible to develop a set of customer damage functions that represent the outage costs customers experience given different combinations of outage characteristics and customer characteristics. The models developed for each customer class provide a more generalized estimate of the average outage cost than can be developed from any of the individual studies because the merged data include a wider range of scenarios and customers.

The models predict that the average cost experienced by an "average" customer for a 1-hour summer afternoon outage is approximately $\$ 2.90$ for residential, $\$ 1,200$ for small commercial and industrial, and $\$ 8,200$ for large commercial and industrial. The outage costs increase substantially, but not linearly, as the outage duration moves from 1 hour to 8 hours. In addition, outage costs are generally higher in the winter than in the summer. The models also demonstrated that there are key differences in outage costs across regions, time of day, consumption, and business types. These models and the associated predicted damage estimates represent the combined average costs across the various studies controlling for other scenario and customer characteristics. They are useful best approximations for outage costs given the underlying characteristics of the samples.

Thus, a noted benefit of this project is the availability of statistical models to be used to calculate outage costs for large C\&I, small-medium C\&I, and residential customers. These models can be applied to geographic regions (not individual customers) for a variety of scenarios.

Table 6-2. Summary of Predicted Outage Costs

\begin{tabular}{|l|rrr|}
\hline & \multicolumn{1}{c}{ Residential } & \multicolumn{1}{c}{ Small C\&I } & Large C\&I \\
\hline Summer Aft -- 1 Hour & $\$ 2.90$ & $\$ 1,200.00$ & $\$ 8,200.00$ \\
Summer Aft - 8 Hour & $\$ 7.20$ & $\$ 4,400.00$ & $\$ 41,000.00$ \\
Winter Aft -- 1 Hour & $\$ 3.30$ & $\$ 1,800.00$ & $\$ 20,000.00$ \\
Winter Aft --8 Hour & $\$ 8.32$ & $\$ 6,300.00$ & $\$ 105,000.00$ \\
\hline
\end{tabular}

While providing a basis for developing a more generalized estimate of outage costs, the models developed from the merged datasets are not perfect. The data available from the various studies used in this meta-analysis suffer from several important limitations. First, there is a certain amount of confounding collinearity across the studies on certain very important variables. For all of the databases, for example, there are "holes" in the data across time, region, and method that make it impossible to disaggregate the affects of these influences. This means that it is impossible to say for certain whether the higher outage cost values for the Southwest, for example, are due purely to their hot summer climate or whether they are higher in part because of the method used to collect the outage cost data in that area or the particular economic and market conditions present during the year that study was done. 
The results also suffer from some degree of collinearity between the regions and the scenario characteristics. The sponsors of these studies generally were interested in testing outage costs for scenarios that met planning needs specific to their systems. Typically these were outages associated with peak demand events or with certain distribution events that produced outages such as lightning strikes or winter storms. As a result, the scenario's tested in any one of the regions tended to represent periods of time when these events were most likely to occur (e.g., summer peaking, or months when thunderstorms develop). Unfortunately, these times are somewhat unique to each study sponsor, so scenario characteristics typically were not repeated identically across the regions. A fortunate characteristic of these studies is that almost all of them included a summer afternoon outage, which provided comparability for that condition.

A second limitation is the lack of coverage for a few regions. The studies that were available tended to cover only certain parts of the country. Of great importance is the fact that no data were available from the Northeast/Mid-Atlantic region and limited data was available for the North Central and Midwest. The Northeast/Mid-Atlantic area is particularly troublesome because it is a unique part of the country in terms of the density of people and businesses. It is possible that the average outage costs from this region may look no different than other parts of the country when weather and customer composition are controlled. But it is equally possible that outage costs have a somewhat different pattern in areas of high density. At this point the available data do not allow us to test for this.

A third limitation is the lack of coverage on a few variables in some of the studies. While most studies included data on most outage attributes, including duration and time of day, a few important attributes, such as whether there was advanced warning, were only tested in one or two studies. Similarly certain potentially important background characteristics were only included in few studies. Variables such as household composition in residential and SIC code in the commercial and industrial databases were included in some studies and not in others. As a result, models that included these variables could not be developed without seriously reducing the available cases for conducting the analysis.

To effectively address these limitations, future work approaching the measurement problem on a supra regional or ideally on a national basis is needed. Attention needs to be given to varying the scenarios across regions and customer classes so as to eliminate some of the collinearity that exists in the present studies, to include a comprehensive set of predictor variables, and to add a few variables that are clearly important but missing from the current studies. Appendix C presents recommendations for improvements to the survey design.

There are several routes that could lead toward an improved understanding beyond that which this study has provided:

1. Encourage all U.S. utilities that have conducted surveys on the economic costs of outages to their customers to contribute these data and thereby enhance the coverage and usefulness of these data on a national scale.

2. Support future utility efforts to collect additional information on the value of electricity reliability toward ensuring that ultimately these data also contribute to improving the 
availability of this type of information on a national basis (e.g., use consistent methodology for survey design and sampling).

3. Where necessary, in order to address existing gaps in available data, lead efforts to conduct additional surveys on topics of high priority (e.g., collect new data in areas of the country or on other reliability issues not currently well-represented in existing data collection efforts or on other reliability topics.

These studies could be conducted with the benefits of lessons from others, ensuring that the methodology would be not just compatible, but synergistic with the current data. In that way a most robust set of data could be used, and periodically replicated for trend analysis. 


\section{References}

Eto, J., J. Koomey, B. Lehman, N. Martin, E. Mills, C. Webber, and E. Worrell (2001). Scoping Study on Trends in the Economic Value of Electricity Reliability to the U.S. Economy. Lawrence Berkeley National Laboratories, Pub LBNL-47911.

Dalton, J.G., III; D.L. Garrison, and C.M. Fallon, "Value-Based Reliability Transmission Planning." Power Systems, IEEE Transactions on , Volume: 11 Issue: 3 , Aug. 1996 Page(s): $1400-1408$

Long, J.S. Regression Models for Categorical and Limited Dependent Variables, Chapter 7 , Sage Publications: Thousand Oaks, CA, 1997.

Sullivan, M. and D. Keane (1995). Outage Cost Estimation Guidebook. EPRI, TR-106082, Research Project 2878-04.

Vojdani, A.F., R.D. Williams, W. Gambel, W. Li, L. Eng, and B.N. Suddeth, Experience with application of reliability and value of service analysis in system planning Power Systems, IEEE Transactions on, Volume: 11 Issue: 3, Aug. 1996 Page(s): 1489 -1496. 
THIS PAGE LEFT INTENTIONALLY BLANK 


\section{Appendix A. Data Transformation}

Creating the meta-datasets involved a multi-step process. First, the datasets, codebooks and survey instruments had to be obtained from the companies if Population Research Systems did not have them already available. Second, datasets had to be standardized and merged. This Appendix describes these processes.

\section{A.1 Acquiring the Datasets}

Companies that had conducted value of service (VOS) studies were contacted by phone by the Project Director. Typically they asked for documentation, so they were emailed a letter and a document explaining the genesis and purpose of the study. These documents are located at the end of this appendix. Two companies declined participation, citing either concerns about legal challenges (although we feel that the existence of the study actually mitigates this risk) or that the study offered their company no value. When requested, Non-Disclosure Agreements were signed assuring that customer-specific information would not be available, an assurance that was actually part of the study design. Because PRS had conducted several of the studies, the data and other materials for those studies were in-house. In other cases we received data files from the utility, or from the consulting firm that conducted the study. In one instance, the data were on 5$1 / 4$ " floppy disks but fortunately they were still readable.

\section{A.2 Construction of the Database}

Altogether, we received 24 different datasets from surveys fielded by 8 different utility companies between 1989 and 2002. Some of the utilities surveyed all three customer types large commercial and industrial (C\&I), small/medium C\&I, and residential - while others did not. In some cases there was only one dataset for commercial and industrial customers, and these were sorted into large or small-medium according to electricity usage. Table A-1 lists the utility company, survey year, and types of data for each of these 24 datasets.

Table A-1. Datasets

\begin{tabular}{|c|c|c|c|c|}
\hline Company & $\begin{array}{c}\text { Survey } \\
\text { Year }\end{array}$ & $\begin{array}{c}\text { Large } \\
\text { C\&I }\end{array}$ & $\begin{array}{c}\text { Small / } \\
\text { Medium } \\
\text { C\&I }\end{array}$ & Residential \\
\hline Southeast-1 & 1997 & \multicolumn{2}{|c|}{ X } & \\
\hline \multirow[t]{2}{*}{ Southeast-2 } & 1993 & $\mathrm{X}$ & $\mathrm{X}$ & $X$ \\
\hline & 1997 & $\mathrm{X}$ & $\mathrm{X}$ & $X$ \\
\hline \multirow[t]{2}{*}{ Southeast-3 } & 1990 & $\mathrm{X}$ & $\mathrm{X}$ & \\
\hline & 1991 & $\mathrm{X}$ & & \\
\hline Midwest & 2002 & $X$ & X & \\
\hline West & 2000 & $X$ & X & $X$ \\
\hline Southwest & 2000 & $X$ & $\mathrm{X}$ & $X$ \\
\hline Northwest-1 & 1989 & \multicolumn{2}{|c|}{$\mathrm{X}$} & $\mathrm{X}$ \\
\hline Northwest-2 & 1999 & $\mathrm{X}$ & $X$ & $\mathrm{X}$ \\
\hline
\end{tabular}


Note: The Midwest company classified the target populations as industrial and commercial rather than large C\&I and small C\&I, as did the other surveys. This distinction did not pose a problem during the standardization process since the companies could be re-apportioned according to $\mathrm{kW}$ demand.

Once received, the next task was to read the datasets, identify the variables required for the analysis, standardize these variables, merge the datasets, and then standardize the dollar amounts into 2002 dollars. The variables required for the C\&I data and Residential data are in Tables A-2 and A-3:

Table A-2. Variables for Commercial \& Industrial Meta-Sets

\section{Outage Specific}

Season

Hour of day

Day of week

Duration

Warning given

Outage cost per event

Disruptiveness rating

Year of survey

Geographic region

Table A-3. Variables for Residential Meta-Sets

\section{Outage Specific}

Year of survey

Season

Hour of day

Day of week

Duration

Warning given

Geographic region

Willingness to pay

Credit (Willing to accept)

\section{Respondent-Specific}

Number of outages

Back-up generator

Peak kW demand

Annual usage

SIC Code

Number of employees

Acceptable service measures

\section{Respondent-Specific}

Housing type and ownership

Sick bed/medical \& med. equipment.

Home business

$\mathrm{HH}$ Income

Number of outages

Back-up generator

Average kW usage

Acceptable service measures 
The small-medium C\&I and large C\&I data required the same variables, so in order to create the small-medium C\&I dataset and the large C\&I dataset, all of the available C\&I datasets, both small/medium and large, were merged together into a single C\&I dataset. The C\&I dataset was then parsed into two portions: small/medium C\&I and large C\&I, based on annual $\mathrm{kWh}$ usage.

A common cutoff point for separating small/medium C\&I from large C\&I is at 1,000,000 kWh annual usage; customers falling below 1,000,000 $\mathrm{kWh}$ of annual usage are considered small/medium C\&I, while those above $1,000,000 \mathrm{kWh}$ of annual usage fall into the category large C\&I. The resulting large $\mathrm{C} \& \mathrm{I}$ dataset $(\mathrm{N}=8,462)$ has all customers with annual usage of $1,000,000 \mathrm{kWh}$ or more; the merged small/medium $\mathrm{C} \& \mathrm{I}$ dataset $(\mathrm{N}=26,444)$ has all customers with annual usage of less than 1,000,000 $\mathrm{kWh}$. The small/medium C\&I dataset is also bounded at $12,000 \mathrm{kWh}$ annual usage. A C\&I customer using less than 12,000 kWh annually would be considered an anomaly. Therefore, we assume that observations with reported usage of less than $12,000 \mathrm{kWh}$ are in error and remove these observations from the merged small/medium C\&I dataset.

As explained in the note at the bottom of Table A-1, the Midwest company's customer base was divided into industrial and commercial customer types, rather than using small-medium C\&I and large C\&I. To conform to the customer types defined in the other datasets, we apply the same decision rule, based on annual $\mathrm{kWh}$ usage, to their industrial and commercial customers, effectively reassigning them as small/medium C\&I or large C\&I.

The combined residential dataset $(\mathrm{N}=42,830)$ is a straightforward merge of the six individual residential datasets.

\section{A.3 Missing Data and Treatment of Outliers - C\&I}

There are three relevant dependent variables in the small/medium C\&I and large C\&I datasets: (1) total outage cost, (2) total outage cost per annual $\mathrm{kWh}$, and (3) total outage cost per peak kW. For the purposes of analysis, there is a different sample size for each dependent variable, based on the number of observations with missing values on the particular dependent variable.

The analysis samples were constructed from the primary C\&I datasets as follows: First, the $.05 \%$ of the sample with the highest values on the relevant dependent variable are deleted from the sample to exclude potential outliers. Second, we eliminate those observations with missing values on the relevant dependent variable. Tables A-4 and A-5 list the resulting number of cases (N) for each analysis sample, for C\&I and Residential, respectively. For example, in the case of the large C\&I, there were 8,462 cases in the dataset, but only 8,418 had data for annual $\mathrm{kWh}$, and fewer $-6,031$ - had data for peak kW. About $7.1 \%$ of cases were excluded owing to outliers and missing data, leaving 7,865 cases available for calculating total cost. 
Table A-4. Sample Sizes of C\&I Analysis Datasets Before and After Deletion of Outliers and Missing Data

\begin{tabular}{|l|c|c|c|c|}
\cline { 3 - 5 } \multicolumn{2}{c|}{} & \multicolumn{3}{|}{$\begin{array}{c}\text { Sample sizes after deletion of missing data and } \\
\text { outliers, by dependent variable }\end{array}$} \\
\hline Customer Type & $\begin{array}{c}\text { Original } \\
\text { N }\end{array}$ & $\begin{array}{c}\text { N for Event } \\
\text { Cost }\end{array}$ & $\begin{array}{c}\text { Nor Cost per } \\
\text { Annual } \mathbf{k W h}\end{array}$ & $\begin{array}{c}\text { N for Cost per } \\
\text { Peak kW }\end{array}$ \\
\hline Large C\&I & 8,462 & 7,865 & 7,821 & 5,724 \\
\hline $\begin{array}{l}\text { Small/Medium } \\
\text { C\&I }\end{array}$ & 26,444 & 23,800 & 23,675 & 11,516 \\
\hline
\end{tabular}

We use the same steps to derive the residential analysis datasets.

Table A-5. Sample Sizes of Residential Analysis Datasets Before and After Deletion of Outliers and Missing Data

\begin{tabular}{|l|c|c|c|}
\cline { 3 - 4 } \multicolumn{2}{c|}{} & \multicolumn{2}{c|}{$\begin{array}{c}\text { Sample sizes after deletion of } \\
\text { missing data and outliers, by } \\
\text { dependent variable }\end{array}$} \\
\hline Customer Type & $\begin{array}{c}\text { Original } \\
\text { N }\end{array}$ & WTP & Credit \\
\hline Residential & 42,830 & 28,042 & 12,615 \\
\hline
\end{tabular}

\section{A.4 Calculation of Total Outage Costs - C\&I}

The calculation of total outage cost varies according to the format of each survey. Some surveys, in addition to asking about total outage costs, ask for detailed estimates of component costs, including lost production/sales, damage to equipment or materials, extra overhead, addition labor and overtime costs, and other costs associated with an outage. Other surveys only request a total estimated cost for each outage scenario.

In cases where both total costs and component costs are available, our estimate of total outage cost is based on the sum of the component costs. However, if the sum of component costs does not match the estimate of total cost provided by the customer, we use the estimate of total cost in our analysis instead of the sum of component costs.

Furthermore, many surveys include multiple scenarios to gather information about outages under different conditions. Outage scenarios may vary by the time of day, day of the week, season, duration of the outage, and whether or not there is advanced warning of the outage. Within our datasets, each scenario is a separate observation. Therefore, each customer may have multiple records within a given dataset, up to a maximum of 6 records for the NW-2 C\&I data. In other words, the scenario became a case to which the individual data were appended. 


\section{A.5 Calculation of Willingness to Pay and Credit - Residential}

The residential surveys do not ask customers for estimates of outage costs because household respondents are unable to accurately gauge the costs unlike business customers. Rather, residential customers are generally asked two questions: (1) how much would you be willing to pay for electric service to avoid the power interruption in the case of this outage (willingness to pay or WTP)? and (2) how much would you accept as a credit for a particular outage scenario (credit)?

These questions can be posed in many ways. Some surveys allow customers to select WTP and credit amounts from a list of possible choices. Others permit customers to enter any amount into a blank field. Many surveys use a combination of methods. For example, the West residential survey asks customers the following questions to determine WTP and credit:

Suppose an electric service was available to handle all of your electrical needs during this Y hour outage. With this service, you would not have to make any adjustments to the outage since your electricity would not go off.

Would you pay $\mathbf{S X}$ for this electric service to avoid this $\mathbf{Y}$ hour outage? (CIRCLE ONE NUMBER)

1 No

2 Yes

-8 Don't Know

-9 Refused/Missing

Would you pay $\mathbf{2} * \mathbf{\$ X}$ for this electric service to avoid this $\mathbf{Y}$ hour outage? (CIRCLE ONE NUMBER)

1 No

2 Yes

-8 Don't Know

-9 Refused/Missing

Would you pay $1 / 2 * \mathbf{\$ X}$ for this electric service to avoid this $\mathbf{Y}$ hour outage? (CIRCLE ONE NUMBER)

1 No

2 Yes

-8 Don't Know

-9 Refused/Missing

What is the maximum you would pay for this electric service to avoid this $\mathbf{Y}$ hour outage?

$\$$ 
-8 Don't Know

-9 Refused/Missing

Our WTP and credit amounts are calculated as the maximum amount provided by the customer. In the case of a categorical response, each category was converted to a numeric value prior to applying the maximization rule.

\section{A.6 Explanatory Variables}

In order to consolidate our 24 datasets into a single dataset for each customer type, we needed to enforce conformity of measures across datasets. Year of survey simply ranges from 1989 to 2002. The region of the U.S. is recoded as: West, Southwest, Northwest, Midwest, and Southeast. Regional assignments are based on the location of the utility company. We do not have any information from the Mid-Atlantic and New England regions, or central to eastern Midwest United States. In the regressions these are separate dummy (dichotomous) variables.

Duration was typically provided as a parameter for each outage scenario. In the 24 datasets, there are 12 different durations, ranging from a voltage sag to a 12-hour outage. Each unique response has been recoded into a new categorical variable capturing the following durations: less than 20 minutes; 21-60 minutes; 61-240 minutes; 241-480 minutes; greater than 480 minutes. In general, these are reported in the study as: less than 20 minutes; 1 hour; 4 hours; 8 hours; greater than 8 hours, respectively.

Most outage scenarios also include the season of the year, day of the week, hour of the day, and whether or not advance warning of the outage is provided. Season has been recoded as a dichotomous variable for winter or summer (no spring or fall scenarios). Day of the week is sometimes specified, although many surveys only distinguish between a weekday and a weekend. Only Friday, weekday, and weekend are represented in these data, so we have collapsed Friday into the category weekday and created day of the week as a dichotomous variable: weekday-weekend. Hour of the day has been collapsed into three categories: morning (6am-12pm), afternoon (1pm-5pm), evening (6pm-12am). No outage scenario uses the hours of midnight-6am. Advance warning of an outage is dichotomized into a Yes/No indicator.

Approximately half of our C\&I observations have a Standard Industry Classification (SIC) code that identifies the primary business of each respondent company. SIC codes are 4 digits. The first digit represents the broadest industry classification and each subsequent digit provides a more granular description of the company's activities. We have recoded SICs into a relatively broad 9-category indicator of industry classification, using the first two digits of each company's SIC codes. Our categories are: manufacturing; agriculture; mining; construction; retail and trade; finance, insurance, and real estate; services; telecommunications and utilities; and public administration.

For our analysis, industry classification has been broken down into 9 general categories, based on Standard Industry Classification (SIC) codes. Each category and its corresponding range of SIC codes is listed in Table A-6. These categories are established using only the first two digits 
of the SIC codes. SIC codes are available in our dataset for $63 \%$ of large C\&I customers and $47 \%$ of small/medium C\&I customers. 


\section{Table A-6. SIC Codes}

\begin{tabular}{|l|l|}
\hline SIC Range & \multicolumn{1}{|c|}{ Industry Category } \\
\hline $01 \mathrm{xx}-09 \mathrm{xx}$ & Agriculture, Forestry, \& Fishing \\
\hline $10 \mathrm{xx}-14 \mathrm{xx}$ & Mining \\
\hline $15 \mathrm{xx}-17 \mathrm{xx}$ & Construction \\
\hline $20 \mathrm{xx}-39 \mathrm{xx}$ & Manufacturing \\
\hline $40 \mathrm{xx}-49 \mathrm{xx}$ & Transportation, Communication, \& Utilities \\
\hline $50 \mathrm{xx}-59 \mathrm{xx}$ & Wholesale \& Retail Trade \\
\hline $60 \mathrm{xx}-67 \mathrm{xx}$ & Finance, Insurance, \& Real Estate \\
\hline $70 \mathrm{xx}-89 \mathrm{xx}$ & Services \\
\hline $91 \mathrm{xx}-97 \mathrm{xx}$ & Public Administration \\
\hline
\end{tabular}

\section{A.7 Dollar Standardization}

Total cost numbers in the small/medium C\&I and large C\&I datasets, as well as credit and willingness to pay (WTP) figures in the residential dataset, were standardized to 2002 dollars using the Consumer Price Index (CPI) from the U.S. Bureau of Labor Statistics (http://www.bls.gov/cpi/home.htm). We used the broadest measure of CPI, calculated for all urban consumers and all items. The base year for this index is 1982-84 (i.e. 1982-84=100). The CPI figure for 2002 is 179.9. For each survey year, we calculated a deflation factor using the formula:

$$
\text { deflation factor }=\mathrm{CPI}_{\text {survey year }} / \mathrm{CPI}_{2002}=\mathrm{CPI}_{\text {survey year }} / 179.9
$$

The final step to standardize our dollar denominated figures - total outage cost, WTP, and credit - to 2002 dollars is to multiply each dollar amount by the deflation index corresponding to the year of the survey. 


\section{Appendix B. VOS Survey Methodology}

With the publication of the Outage Cost Estimation Guidebook, survey protocols for gathering these data were developed and generally followed by the various firms conducting Value of Service (VOS) studies. The methodology varies somewhat for each customer group, and each will be summarized in this appendix.

\section{B.1 Survey-Based Methods of Cost Estimation}

The studies used to create the meta-database in this project employed a survey-based methodology to gather information about the value of reliable service. The results allow for the development of estimates of outage costs. There are two forms of estimates - direct cost (or worth) and imputed cost estimation. Direct cost is more typically used for non-residential customers, whereas the imputed cost is used for residential customers because many of the costs to residential customers are of an intangible nature, whereas the costs to businesses typically are quantifiable.

\section{B.1.1 Direct Cost Estimation}

With the direct measurement approach, the survey describes hypothetical outage "scenarios" that have different characteristics. Each outage scenario describes a specific combination of characteristics making up one outage event. Characteristics that are varied include:

- The season in which it occurs (summer and winter).

- The day of the week (weekend versus a week day).

- Start time.

- Duration.

- Complete or partial loss of service (voltage sag or black-out).

- Voluntary or mandatory.

- Amount of advance warning, if any.

Respondents will usually receive several scenarios. However, because the utility often wants to explore more scenarios that respondents can reasonably expect to have time or patience to answer, there are typically several versions with a questionnaire, each having three to five scenarios. An example of such a scenario is:

At 1:00 PM on a summer weekday, the electric power serving your business stops without warning. You don't know how long this power outage will last when it occurs. After one hour your power comes back on.

Then the commercial and industrial customers are asked to estimate the costs, damages, and if relevant, savings accrued from each outage. They are given a worksheet to fill out which would look something like this:

For this outage, estimate costs from:

Damage to equipment:

Damage to materials:

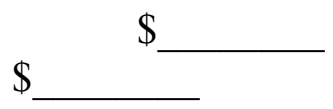


Wages paid without production:

Other costs:

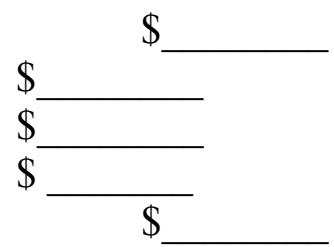

Less:

Lost sales (or production):

Percentage of sales to be recouped: \% x Sales lost

Total sales lost:

Wages saved:

Energy costs saved:

Other savings:

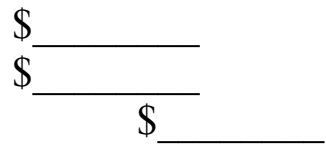

Total Costs:

$\$$

\section{B.1.2 Cost Estimation Through Imputation}

Willingness to pay and willingness to accept credit (WTP and WTA) approaches instead ask the customer what they would pay to avoid the outage occurrence, or how much the customer would have to be compensated to be indifferent to the outage. As with the direct cost approach, the survey describes hypothetical outage "scenarios" that have different characteristics. The imputed approaches are especially useful in situations where intangible costs are present that are difficult to estimate using the direct worth approach, which is typically the case for residential customers. Because not all surveys used the WTA measure, the meta-analysis employed only WTP. A full discussion of the advantages and disadvantages of the direct worth and imputed methods can be found in Chapter 3 of the Outage Cost Estimation Guidebook.

The example below is from a mail survey.

Case \#1: On a summer weekday, a power outage occurs at 3:00 PM without any warning. You do not know how long the power outage will last, but after 1 hour your household's electricity is fully restored.

Willingness to Accept Credit Imputation:

Suppose your Utility could provide you with a credit on your bill each time your home experienced this outage, whether or not you were home (LL!?). What would be the least amount that you would consider a fair payment for each time this outage occurred in your home? (Circle or enter a number)

$\begin{array}{lllllllllll}\$ 0 & \$ .10 & \$ .25 & \$ .50 & \$ 1 & \$ 2 & \$ 3 & \$ 4 & \$ 5 & \$ 6 & \$ 8 \\ \$ 10 & \$ 12 & \$ 15 & \$ 20 & \$ 25 & \$ 30 & \$ 40 & \$ 50 & \text { Other: } \$ & \end{array}$

Willingness to Pay Imputation:

Suppose a back-up service was available to handle all of your household's electrical needs during this power outage. You would be billed by the supplier only for when and for how long the back-up service provided you with electricity. If you were charged a fee for this service only when you decided to use it (by using an on-off switch in your home), 
what is the most you would be willing to pay for this service each time you used it to avoid this power outage? (Circle or enter number)

$\begin{array}{lllllllllll}\$ 0 & \$ .10 & \$ .25 & \$ .50 & \$ 1 & \$ 2 & \$ 3 & \$ 4 & \$ 5 & \$ 6 & \$ 8 \\ \$ 10 & \$ 12 & \$ 15 & \$ 20 & \$ 25 & \$ 30 & \$ 40 & \$ 50 & \text { Other: } \$ & \end{array}$

An alternate version of a WTP question when fielded by telephone is:

Suppose an electrical service was available to you during the power outage. With this service, you would not have to make any adjustments to the outage since your electricity would not go off.

Would you pay $\$ 10.00$ for this service to avoid the outage? (YES or NO)

[IF YES]: Would you pay $\$ 20.00$ for this service?

[IF NO]: Would you pay $\$ 5.00$ for this service?

In general, however, it is ideal to conduct this kind of research using mailed survey instruments, although it's possible a combined mixed mode mail-Internet methodology may now be reasonable.

\section{B.1.3 Survey Design}

As is typical, the analysis is conducted based on actual usage, hence groups into 'large' or 'small-medium' commercial and industrial. In reality, the survey instruments may be designed to ask questions that are relevant to different companies given their primary mode of business. Manufacturing companies are asked about production and materiel costs, damages and savings resulting from outages to their resources, equipment, and labor. Retail and commercial organizations are asked about the impact of power loss on sales and inventory. A few studies have included other subgroups, such as agricultural customers, hospitals, and service organizations. In the meta-database, we excluded these latter categories due to an inadequate number of cases.

\section{B.2 Data Collection Methodology}

\section{B.2.1 Non-Residential Customers}

Survey instruments for outage cost studies are complex and difficult to answer. For very large organizations, it is best to have a mid-level to senior-level analyst or consultant conducting the interview on-site. This interview takes approximately 2 to 4 hours, and can include input from more than one departmental manager. Sometimes several persons will be interviewed together, and other times sequentially. Answers required for the survey are not likely to be known 'off the top of one's head' nor would they be reliable if given as such. Therefore, the process is a "phone-mail-interview" technique, where the research organization is given the initial list of company and contacts, the correct respondent(s) is identified in an initial phone call, and an onsite interview is then scheduled. The respondent is then mailed or faxed the survey instrument with instructions, so that this information will be available at the time of the on-site interview. 
The presence of the interviewer ensures that the respondent has a clear understanding of how to interpret the survey requirements.

A less expensive variation of this procedure is "phone-mail-phone" where instead of conducting the interview on-site, the interview is conducted over the phone. This methodology may be appropriate for the small-medium organizations. Finally, there have been low budget projects where the account contact was sent the survey by mail and then returned it. With follow-up, such as reminder postcards and other best practices in mail surveys, this method may have a reasonably high response rate but the data quality tend to be compromised by this methodology.

\section{B.2.2 Residential Customers}

There is much less of a respondent recruit issue for residential customers. This survey is also conducted by mail, using best practices for mail surveys to garner a high response rate.

Residential surveys can also be conducted by telephone. There are certain implications about questionnaire design (such as the way Willingness-to-pay questions can be asked) for each methodology. 


\section{Appendix C. Recommendations for Questionnaire Design}

One of the benefits of conducting this meta-analysis is revisiting the questionnaire design and the data analysis made possible by these survey instruments. Reviewers of an earlier version of this document also noted that improvements to methodology could be made.. Therefore, should a utility, Public Utilities Commission, a federal agency or other organization choose to conduct a Value of Service (VOS) study, it is worthwhile to consider the lessons learned along the way. Certainly, studies conducted by utilities need to address that utility's specific operating environment and customer mix. Nevertheless, there are some practices that could not only provide the utility with better data, but also allow for future meta-analyses and contributions to a wider industry understanding of the value customers place on reliability. These practices are summarized in this Appendix.

\section{C.1 Macro- Versus Micro-Views}

The customer groups presented in this research included households, businesses, and manufacturers. While some utilities branched out to a more diverse set of businesses, manufacturers or producers, such as agricultural or healthcare organizations, no study included the broad impacts of an outage on societal or government costs. Some of those costs would understandably be more difficult to quantify, but others can be captured in dollars. For example, governments lose sales tax revenue, and may need to expend emergency dollars for police or other security measures. A government office does not lose sales revenue, but it does lose productivity in the form of staff that gets paid regardless, or fees for government licenses and services that go uncollected. Future studies are advised to branch out to these non-business outage costs.

\section{C.2 The Impact Of Back-Up Systems}

Through the course of analysis of the different survey instruments, it became obvious that the meaning and implications of having a back-up generation system were not consistently captured in the survey methodology. In these questionnaires, respondents were asked at one point in the survey whether they have a back-up generator or system, and then only later answered the scenario-specific questions. Two problems are inherent in the question about back-up systems. First, the precise kind of back-up system was not necessarily clarified, for example, was it just for lighting, or was it for full operations? Second, the presence of the generator and the tally of outage costs were separated, so it was not clear if the respondent was adequately taking the backup generation capability or costs into consideration.

\section{C.3 Advance Warning}

In the studies employed in this meta-analysis, scenarios with advance warning were not necessarily paired with the identical scenario (and company-respondent) without advance warning, so the aggregate analysis yielded highly problematic or counter-intuitive results. The implication of this methodological problem is that it will be difficult to compare the costs of transmission to generation outages. 


\section{C.4 Facilitating Regional Comparisons}

Being able to compare the results of one study to another are important for an individual utility as well as for cross-service territory insights. There are several techniques in survey design or database design, that would facilitate this kind of analysis. These are:

- Noting regional climates in a standardized nomenclature.

- Including standard outage scenarios, such as, by including one-hour summer afternoon weekday for C\&I, and one-hour winter morning weekend for residential customers.

- Standardization of costs and savings calculations in the commercial and industrial surveys, and scales for asking willingness to pay and willingness to accept credit questions for the residential surveys.

- Noting whether the location is urban, suburban or rural.

Many organizations and industries have standardized protocols (such as quality) in order to have a better understanding of benchmarks, trending and best practices. Standards to VOS studies would go a long way in ensuring comparability across time and territory.

\section{C.5 Commercial and Industrial Classification Codes (NAICS or SIC)}

More help needs to be provided to respondents in answering this question, such as a brief summary next to a check-box for the code so at the very least, they can get the correct top-level classification. Yet even using a precise Industrial Classification code has its limitations. A retail company that gets the bulk of its business on weekdays from 9 to 5 with from customers in the store is going to have a different reaction to an outage than an establishment that does $75 \%$ of its business in the evenings, or during Friday to Sunday (e.g., movie theatres). A professional services firm that relies on electronics and telecommunications equipment comes to a standstill, while another has activities that can be accomplished without power. While some instruments do note the regular business hours, the information about the kind of business needs to be standardized for ease of analysis and cross-comparison.

\section{C.6 Residential Costs and Presence at Home}

In some cases, household respondents were asked to input their willingness to pay or accept credit for outages regardless of whether they were home. Yet a debate around the meaning of costs for residents hinges on whether they are home, and how much of the cost of an outage is due to cessation of household activity, and how much is due to impact on household appliances and electronics. Indicating whether the respondent is normally at home during the time of the outage scenario would add clarification.

A second aspect of residential costs is the use of the WTP and WTA measures. Including both measures in the survey, and using an average of the two for each scenario, would be a further step in the standardization of the survey instrument and the analytic procedure. 


\section{Appendix D. Supporting Documents}

\section{D.1 Cover Letter To Utilities}

Dear $<>$ :

The purpose of this note is to provide information behind our request of the VOS datasets conducted by XX. The US Department of Energy is asking the XX to support an important national research project being carried out by Lawrence Berkeley National Laboratory. The objective of this research project is to assemble a comprehensive database of studies describing the economic value of electric service reliability in the US; and from that database to derive realistic interruption cost estimates for key elements of the national grid.

This database will be created by analyzing the interruption costs reported by electric customers throughout the nation during the 1980s and 1990s. The information required to create this database has already been collected for most of the US in a number of pioneering studies carried out by major utilities. These studies, known as "Value of Service" studies, were carried out by PG\&E, BPA, Southern Company, Duke Energy, Southern California Edison, Niagara Mohawk, Florida Power Corporation, Puget Sound Energy and the Salt River Project. Since virtually identical statistical survey methodologies were used in all of these studies, it is possible to combine the results to obtain important information about interruption costs for different types of customers in various climates and economic contexts. I am asking all of the above utilities to participate in this research effort by contributing the results of the studies they have completed to date.

In particular, we are asking that XX provide to the Laboratory results of economic surveys it carried out in the 1980s and early 1990s to measure the economic value of electric service reliability to customers in its service territory. The information provided by XX will be stored in a secure environment and will not be revealed on a utility or customer level outside the context of the research team. Once the combined database has been assembled, the original data and documentation from the utilities will be destroyed or returned depending on your preference. Very little effort should be required to supply the information we are seeking. The team needs nothing more than digital datasets from the studies and the documentation required to interpret them.

We cannot reimburse you for the cost you incurred in carrying out these important studies, but we are prepared to work with your staff to define a useful information product that can be obtained by combining the results from the various studies. There are a number of possibilities that may be of interest to your staff. These include:

1. Preparing updated interruption cost estimates for the XX service territory based on trends in interruption costs observed for the participating utilities; and

2. Market segmentation analysis designed to identify customer segments with relatively high requirements for power supply reliability and quality. 
We have retained Dr. Michael Sullivan of Population Research Systems (a member of the FSC Group) in San Francisco to assemble and analyze the database. He will be calling you soon in order to facilitate progress. I have taken the liberty of attaching a more detailed description of the research project. Please pass it along to the appropriate parties in your organization, encourage them to listen to our proposal and, if at all possible, participate in the research.

Sincerely,

Leora Lawton, PhD

Consultant

FSC Group 


\section{D.2 Project Background Document}

\section{Project Description}

\section{National Grid Interruption Cost Estimation Project}

\section{Background}

Since the mid-1990s, there have been a number of important changes to the institutional and regulatory framework within which electric service is provided in the United States. Prior to 1996, electric utility companies operated their transmission systems exclusively for the benefit of their customers and shareholders. However, in April of 1996, under FERC Order 888, electric utilities were required to provide open access to generators transmitting electric power into and through their service territories, and interstate wholesale electric power markets subsequently emerged in most regions of the country. As a consequence of FERC Order 888, regulatory authority over transmission pricing and investment planning shifted from State governments to the FERC in virtually all states. More recently, electric utility companies that own and operate independent electric transmission systems are being required (under FERC Order 2000) to relinquish operational control or ownership of their facilities to Regional Transmission Operators - sometimes retaining responsibility for reliability investments and sometimes not.

Sweeping changes were also made in the regulatory structures of a number of retail electricity markets in the same time period - most notably in California, Pennsylvania, Massachusetts and Texas. Together these changes in the wholesale and retail markets have lead to the deintegration of a number of vertically integrated electric utilities into stand-alone companies each separately responsible for distribution, transmission and generation. With the de-integration of these formerly vertical monopolies, centralized operational functions such as unit commitment, maintenance scheduling, security and stability coordination and reliability planning have been transferred outside the span of control of utilities and in at least one case virtually replaced by "market mechanisms." In the context of these changes, centralized demand-side management functions designed to shed loads or limit growth in demand and consumption have been significantly scaled back as companies operating in competitive markets have become increasingly focused on price differentiation to retain market share.

The de-integration of centralized command and control functions for system operations, reliability planning and demand-side management has resulted in near catastrophic degradations in service quality and reliability in some cases. The poster child for what can go wrong in the electric supply system when these functions de-integrate is California. However, there have been other less dramatic failures including the Western states outage of 1996, and the large-scale distribution system outages in the Northeast and Midwest during the summers of 1998 and 1999.

It is obvious that the efforts to restructure wholesale and retail markets to date have not been very successful. However, no thinking person would advise a return to the situation that led to industry restructuring, in part because it is impossible and in part because the previous industry structure was producing its own, albeit less serious economic problems. However, it is clear that going forward, carefully designed public policies that reestablish electric system security and reliability and encourage cost-effective reductions in demand and consumption must be developed in the context of the restructured industry. A critical requirement for achieving both 
of these objectives is an understanding of the economic consequences of electric service unreliability and the role that demand-side management programs can play in fostering efficient infrastructure investments.

Common sense and a considerable amount of empirical evidence indicate electric utility customers incur substantial economic costs as a result of electric service reliability problems. In the recent past, these costs have not been accounted for in a number of important decisions made by regulators and policymakers in wholesale and retail markets leading to the astonishingly serious consequences experienced by California during the winter of 2000. Given the widening geographical scope of the Federal government's regulatory authority and its influence on regional wholesale electricity markets, and the de facto disintegration of many major electric service companies, it is vital that Federal regulators systematically consider the economic cost of the reliability impacts of future policy making regarding wholesale market structuring and encourage State government regulators to adopt reasonable policies designed to ensure utilities under their regulatory authority are economically viable and make prudent investments in reliability and demand-side management.

Unfortunately, accurate information concerning the economic impacts of electric service unreliability is very limited. After a thorough review of the available literature on the cost of reliability and power quality, the Energy Analysis Department of the Energy Technologies Division of the Lawrence Berkeley National Laboratory concluded ${ }^{4}$ :

1. There are few estimates of the aggregate cost of unreliable power to the U.S. economy; and the estimates that are available are undocumented or based on questionable assumptions.

2. Costs of large-scale outage events (e.g., State- or region-wide power outages) are not well documented and mostly based on natural disasters for which it difficult to separate costs of electric interruptions from damages caused by other disaster features (e.g., property damage from wind or water).

3. Studies of hypothetical outages obtained from outage cost surveys could be used to prepare aggregate estimates of outage costs. However, there are important differences in the survey and statistical methodologies used in the studies that are available in the public domain and these differences prevent rigorous meta-analysis.

4. Very little information is available in the public domain regarding the costs of power quality problems - an increasingly important aspect of service reliability.

In response to the above conditions, the LBNL Scoping study recommended the following actions be taken:

1. The database on outage and power quality event costs should be extended to include information from older assessments and numerous unpublished studies that have been carried out over the past 20 years by utilities and other entities.

\footnotetext{
${ }^{1}$ See: "Scoping Study on Trends in the Economic Value of Electricity Reliability to the U.S. Economy," by Joseph Eto, et al., LBNL-47911, June 2001
} 
2. Meta-analysis techniques should be used to synthesize and better understand the limitations of extrapolating information in the outage cost database with the objective of developing comprehensive national estimates of interruption costs and related subanalyses.

3. Information on the costs of outages and power quality events should be integrated with information concerning the frequencies of the occurrence of such events.

4. Additional information should be collected on the cost of power quality events.

5. Common guidelines for future interruption cost survey information and participation in the development of new primary survey information needed to address key empirical gaps in information concerning the cost of outages and power quality problems should be considered.

This project is designed to accomplish the first two of the above objectives. That is, it will assemble a comprehensive database of studies describing the economic value of electric service reliability in the U.S., and from that database it will derive realistic interruption cost estimates for key elements of the national grid. 


\section{D.3 Project Objectives}

\section{D.3.1 Extend Database On Costs of Outages and Power Quality Problems}

Perhaps the most promising untapped source of reliable information concerning customer interruption costs and costs of power quality events is found in the customer interruption cost surveys that have been carried out by the electric utility industry during the past 15 years. Most of these studies employed a common survey methodology including sample designs, measurement protocols, survey instruments and operating procedures. This methodology is described in detail in EPRI's "Outage Cost Estimation Guidebook." Utilities that used a variant of the approach outlined in the Guidebook include the following:

- Southern California Edison Company (1987 and 2000)

- Pacific Gas and Electric Company $(1986,1987,1989,1993,1996)$

- Southern Company $(1987,1999)$

- Niagara Mohawk (1985)

- Duke Energy Company $(1992,1997)$

- Bonneville Power Administration (1987)

- Salt River Project (2000)

- Puget Sound Energy (1999)

- Cinergy (1998)

- An unnamed Florida utility (1987)

The areas that can be represented by the above utilities include virtually the entire Southeast, most of the Western U.S. (including almost all of California, rural Washington and Oregon and the largest metropolitan areas in Arizona and Washington), the Midwest south and east of Chicago and the Northeast (principally rural areas). The time frame covered by the studies ranges from as early as 1985 to as late as 2000. In several major studies (e.g., Southern California Edison, Pacific Gas and Electric, and Duke Energy) the same customer classes were surveyed using virtually identical survey instruments at different points in time - sometimes separated by as much as 10 years. There are also studies in which interruption costs of similar customer populations (e.g., residential customers) were observed roughly at the same time using nearly identical measurement protocols for utilities located in different places (e.g., Southern California Edison 2000 v. Salt River Project 2000 v. Puget Sound Energy 1999). In almost all of these cases, detailed demographic and firmographic information was collected from study respondents and incorporated into an eventual digital database of results.

In addition, several of the above studies specifically focused on measuring the economic costs of power quality disturbances for large commercial and industrial concerns (i.e., Duke Energy, Southern Company, Cinergy and Salt River Project). The studies carried out for Cinergy and Salt River Project collected both direct cost estimates of power quality disturbances and cost estimates using hedonic methods (e.g., Willingness-To-Pay experiments).

\footnotetext{
${ }^{5}$ See: "Outage Cost Estimation Guidebook," by Michael Sullivan and Dennis Keane, Electric Power Research Institute, EPRI TR-106082, December 1995
} 
The number of studies, span of time and wide geographical scope of the prior research make it an extremely valuable source of information on customer interruption costs arising from all kinds of reliability problems. By making simple and reasonable assumptions (about the generalizability of existing survey findings to adjacent geographical areas), the results from these studies can be extended to the nation and major national grid regions.

\section{D.3.2 Synthesize Customer Damage Functions}

The combination of the data from previously described studies can support a wide variety of analyses needed in the development of useful aggregate and regionally disaggregated estimates of customer interruption costs. Some examples of these analyses include:

1. Development of robust customer damage functions (relating event duration to cost) for specific types of customers (i.e., residential customers, commercial and industrial customers and for business customers by SIC code grouping) ${ }^{6}$. These functions are the mathematical foundation upon which realistic estimates of aggregate customer interruption costs are made.

2. Analysis and description of the variation in interruption costs arising from customer characteristics such as business type (i.e., SIC Code groupings) and number of employees for businesses and family structure, income and number of household members for residential customers. Sample sizes in studies carried out by individual utilities have tended to be between 1,000 and 1,500 for residential customers and 1,200 to 1,500 for commercial and industrial customers. These sample sizes are too small to permit precise measurement of customer interruption costs within customer groupings. However, in grouping the surveys from the 10 different utility firms, sample sizes from the individual studies will be multiplied by a factor of 10 . This means that relatively subtle effects of customer characteristics can be detected and reported. If significant differences are found by customer type and size (and I believe they will be), then customer damage functions can be derived for these sub-groupings.

3. Description of the changes in customer interruption costs that correspond with the passage of historic time. Two interesting issues can be addressed by such an analysis. One issue concerns the description of secular trends in interruption costs arising from changing technological conditions. Where multiple measurements have been taken for the same customer populations at different points in time and macro-economic conditions are not significantly different (this is true for measurements taken in 1987 and 2000 for the SCE study), it is possible to describe the rate at which interruption costs are increasing or decreasing as a function of technological change. The other issue concerns description of the sensitivity of interruption costs to changing macro-economic conditions. Where multiple measurements have been taken under varying macroeconomic conditions (i.e., in expansionary vs. recessionary periods), it should be possible

\footnotetext{
${ }^{6}$ For a detailed discussion of the development and use of customer damage functions see: "Prediction of Customer Load Point Service Reliability Worth Estimates in an Electric System," by Lalit Goel and Roy Billinton, 1994, IEE Proc - Gener. Transm. Distrib, Vol 141, No. 4, July 1994
} 
to detect the effect of the macro-economic conditions. This occurred for PG\&E measurements taken in 1987 and 1993 for residential customers.

4. Description of the variation in interruption costs corresponding with changes in geographic location, population density and climatic conditions. Climatic variation has a powerful influence on interruption costs experienced by residential and commercial customers - less so for industrial concerns. In locations that experience extremely hot summer days, interruption costs may be more than twice as high as they are for more temperate locations. By combining the information from the various surveys, it should be possible to observe the differences in interruption costs that result from difference in climate, controlling for customer type, size and historical time.

The result of this work will be a series of technical articles describing interruption costs derived from the meta-analysis of the survey data provided by the participating utilities. At a minimum, a detailed technical report will be prepared describing customer damage functions (i.e., average interruption costs per event for duration ranging from 1-2 cycles to 8 hours) for the nation as a whole and for specific national grid areas:

- $\quad$ by customer type (i.e., residential, commercial and industrial);

- by major business type categories (e.g., office, dry good retail, light industrial, hightechnology, heavy manufacturing, etc.); and

- $\quad$ by household characteristics (e.g., income levels, family structure, home ownership, etc.).

\section{D.3.3 Benefits to Utility Stakeholders}

The reliability of the electric grid is an important national asset that has been undervalued with tragic consequences in recent policymaking by Federal and state government authorities. In the meantime, efforts to restructure the electric utility industry continue. For example, the U.S. Senate recently passed the National Energy Bill containing provisions establishing a new mechanism for ensuring the reliability of the national grid. Will this and other initiatives properly account for the economic value of service reliability in the future? It is difficult to imagine that it will in the absence of reliable information about interruption costs.

On one hand, the electric utility business is much more competitive than it was 10 years ago. On the other, there are certain issues facing the industry in which their joint interests far outweigh competitive considerations. One of those issues is the economic value of service reliability. Possession of information regarding customer interruption costs conveys little, if any, competitive advantage to a single utility. While combining this information from all major utilities offers significant potential for improving the quality of public policy affecting the industry. All parties in the electric utility industry will benefit if the value of service reliability is appropriately considered in future policy making by state and Federal authorities. It is also likely that all parties in the electric utility industry will lose if this information is not considered in future policy making. 
In addition to industry-wide benefits, the project will benefit the individual utilities that contribute information to the effort. The specific benefits will be negotiated on a case-by case with participating utilities. Examples of benefits that may be provided include but are not limited to the following:

1. Preparation of updated interruption cost estimates for the specific utility service territory based on trends in interruption costs observed for the participating utilities over time;

2. Estimation and delivery of utility specific customer damage functions for different types of customers;

3. Market segmentation analysis designed to identify customer segments with relatively high requirements for power supply reliability and quality; or

4. Other custom analyses and reports that may be derived from the combined database.

\section{D.3.4 Analysis of Risks to Utility Stakeholders}

The risks involved in sharing the requested information are minimal. The primary risks that might arise from sharing the requested information fall into two categories. They are:

1. Competitive disadvantage arising from release of detailed information regarding customer interruption cost information for the utility and/or its customers; and

2. Economic loss arising from the use of the information in litigation related to customer losses experienced during outages; and

Each of these risks is discussed below.

\section{D.3.5 Competitive Disadvantage}

The data supplied by each utility will be kept in a secure database that can be accessed only by members of the project team. All members of the project team will sign confidentiality agreements stating that they will not under any circumstances divulge information containing utility or customer identifiers. The raw data from utilities will be kept under lock and key while it is in the possession of the research team, and staff will only have access to the information at the research facility (e.g., it will not be removed from the facility to work at home). The working database will be accessible by the project team via a local area network at the research facility. This network is located behind hardware and software firewalls to prevent intruders from gaining access.

The project has no need for information regarding the identity of customers who participated in the original studies. This information is not being requested, and if provided, it will be stripped from all files before they are incorporated into the project database. To prevent inadvertent disclosure of proprietary information on a going forward basis, the final project database will not contain the identities of the utilities that provided the interruption cost measurements. Instead, measurement records will be classified by customer type (Residential, Commercial or Industrial), business type (SIC code), national grid region, population density classification (i.e., Urban, 
Suburban or Rural), and other variables that cannot be directly linked back to the participating utilities. Once the final project database has been assembled, all records containing utility identification will be destroyed along with any link tables that may have been used during the course of database development.

Given the foregoing design and operational security measures, we believe there is very little risk that proprietary information will be purposefully or inadvertently released during the data integration phase of the project. Moreover, since the final project database will not contain utility identifiers, there is virtually no chance that the final database can be used to the utilities' disadvantage.

\section{D.3.6 Risk from Litigation}

Customers sometimes bring legal actions against utilities attempting to recover economic losses resulting from service interruptions. Historically, these actions have been brought by individual parties, but a few class actions have been filed in recent years. In a case brought by an individual, three things must be proven. They are:

1. Defendant was negligent;

2. Plaintiff sustained damages (usually monetary in nature); and

3. Damages equaled some provable economic loss.

Results from interruption cost surveys cannot be used to prove that a given party sustained damage during an outage or quantify the magnitude of their economic loss. The primary reason this is so is that interruption cost surveys do not measure the damages and economic losses experienced by plaintiffs in the matter at hand. They measure damages and economic losses for a statistical population that may be more or less similar to plaintiffs along any number of dimensions. As such, they are nothing more than interesting facts that are simply irrelevant to the issues that must be proven in a tort. In a tort, along with proving negligence, plaintiffs must show they were damaged and provide proof of their economic losses; not that the average person would have been damaged and suffered a certain economic loss on the average. Information from interruption cost surveys is useful for proving the latter proposition, but not the former.

In class actions, where the existence of numerous plaintiffs makes the use of statistical methods almost a necessity, legal scholars have been more liberal in their adoption of statistical surveys in finding damages and assessing their economic worth. In these cases, plaintiffs have the added burden of showing that there is a commonality of exposure and injury among class members that justifies treating all the members of the class alike. If anything, results of interruption cost surveys provide strong evidence that: (1) it cannot be presumed that interruption of electric power necessarily damages all customers, and (2) costs from power outages vary widely with circumstances, with seemingly similar customers experiencing little or no loss while others suffer serious losses. In effect, these studies show that some parties sustain damages and losses under circumstances when other similarly situated parties do not. These facts lend support to the notion that insufficient commonality exists among electric customers to justify class certification. To date, there have been no successful class action lawsuits brought by electric customers in 
matters related to service reliability because courts have generally refused to certify classes because of lack of commonality.

\section{D.4 Description of Information Requested From Utility Participants}

The information that is being requested from participating utilities is as follows:

1. A copy of digital data files describing on a case-by-case basis the results of interruption cost surveys carried out during the1980s and 1990s (data may be supplied on any media in any standard format including SAS, dBase, MS Access, text, CSV, MS Excel or STATA);

2. Documentation of data files including variable names, locations (when appropriate and necessary) and field descriptions;

3. Consultant reports summarizing sampling, research methods and data cleaning conventions; and

4. Consultant reports summarizing the results of the study.

Information concerning the identities of the customers involved in the study is not desired and will not be used in the study. However, for statistical purposes, information concerning customer SIC code, geographical location, annual consumption and maximum demand are highly desirable. This information is also requested for each customer that was studied. In most cases, this information will have been attached to the digital files summarizing responses to the survey. However, in some cases, this information may have been kept in separate files. In the latter case, please supply copies of these files along with the information requested above. 